Aus dem Institut für Allgemeinmedizin

(Prof. Dr. med. E. Hummers-Pradier)

der Medizinischen Fakultät der Universität Göttingen

\title{
Ethikberatung in der ambulanten Versorgung
}

Eine Befragung von Hausärzten zur Häufigkeit ethischer Konflikte und zum Beratungsbedarf

\author{
INAUGURAL - DISSERTATION \\ zur Erlangung des Doktorgrades \\ der Medizinischen Fakultät der \\ Georg-August-Universität zu Göttingen
}

vorgelegt von

Konstantin Kallusky

aus

Hannover

Göttingen 2017 
Dekan:
I. Berichterstatter:
Prof. Dr. disc. pol. W. Himmel
II. Berichterstatter/in:
Prof. Dr. Alfred Simon
III. Berichterstatter/in:
Prof. Dr. Thomas Meyer

Datum der mündlichen Prüfung: 21.09.2017 
Hiermit erkläre ich, die Dissertation mit dem Titel „Ethikberatung in der ambulanten Versorgung - Eine Befragung von Hausärzten zur Häufigkeit ethischer Konflikte und zum Beratungsbedarf" eigenständig angefertigt und keine anderen als die von mir angegebenen Quellen und Hilfsmittel verwendet zu haben.

Göttingen, den 


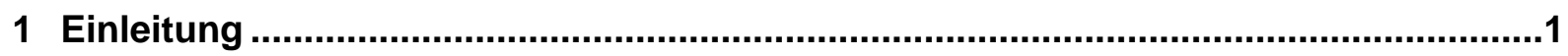

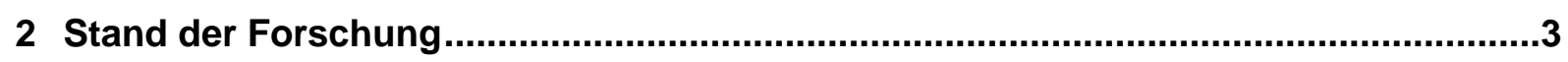

2.1 Beginn und Entwicklungen der Medizinethik in Amerika ...................................... 5

2.2 Beginn und Entwicklungen der Medizinethik in Deutschland ................................ 6

2.3 Aufgaben und Ziele der klinischen Ethikberatung ......................................... 7

2.4 Formen, Ablauf und Organisation klinischer Ethikberatung ................................... 8

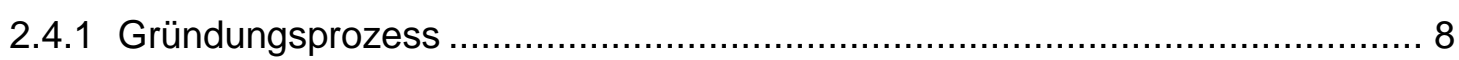

2.4.2 Allgemeine Grundsätze einer klinischen Ethikberatung ............................. 9

2.4.3 Beratungsmodelle und Konzepte der Klinischen Ethikberatung ..................... 9

2.4.4 Ablauf einer klinischen Ethikberatung …........................................... 10

2.5 Anforderungen an einen klinischen Ethikberater ...............................................11

2.6 Sichtweisen und Nutzung klinischer Ethikberatung durch medizinisches

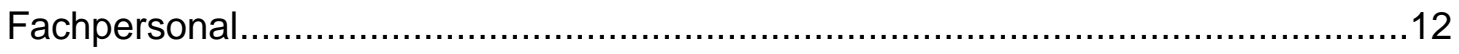

2.6.1 Gründe für die Inanspruchnahme einer klinischen Ethikberatung .................13

2.6.2 Gründe gegen die Inanspruchnahme einer klinischen Ethikberatung.............13

2.6.3 Gegenwärtige klinische Ethikberatung in Deutschland...............................14

2.7 Ethikberatung in der ambulanten Gesundheitsversorgung...................................15

2.7.1 Angebote einer Ethikberatung für Allgemeinärzte ....................................16

2.7.2 Spezifika der allgemeinärztlichen Praxis im Unterschied zum Haus der Maximalversorgung …................................................................ 17

2.7.3 Schwierigkeiten einer Etablierung der Ethikberatung für Hausärzte...............20

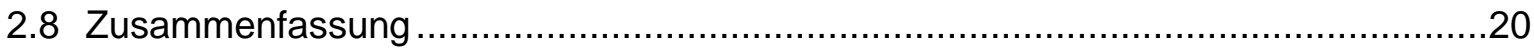

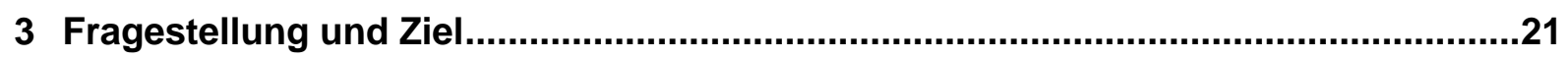

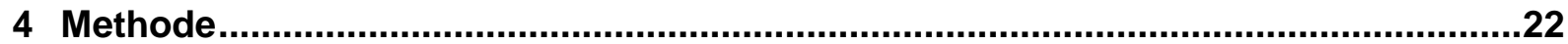

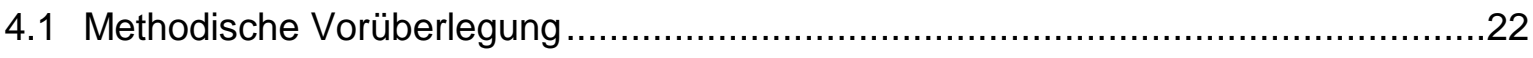

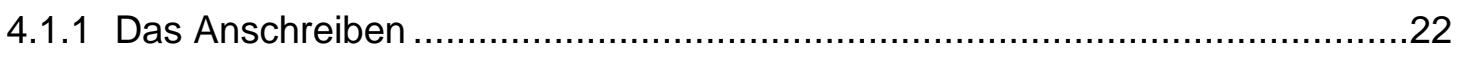

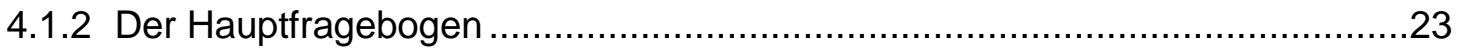

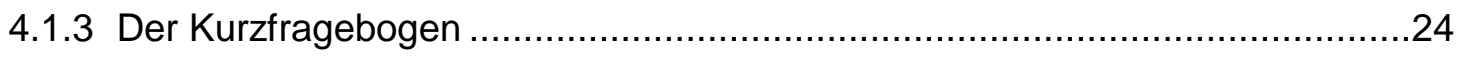

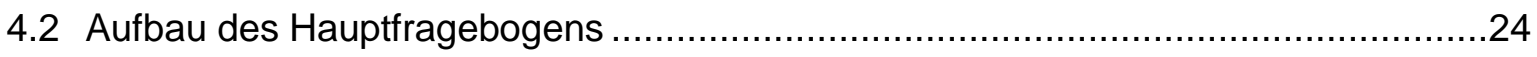

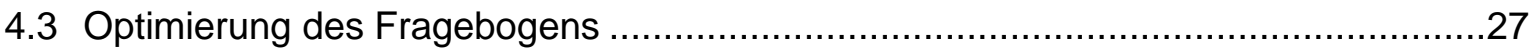




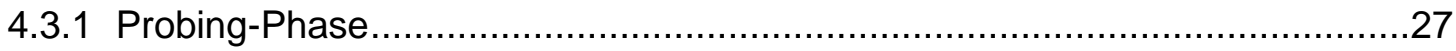

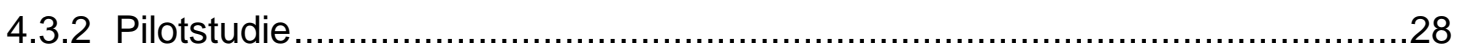

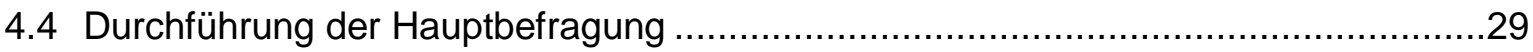

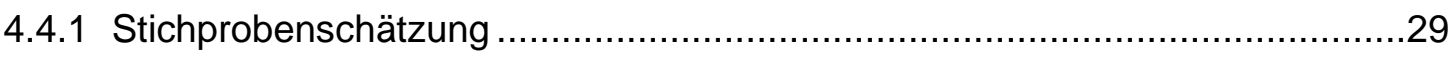

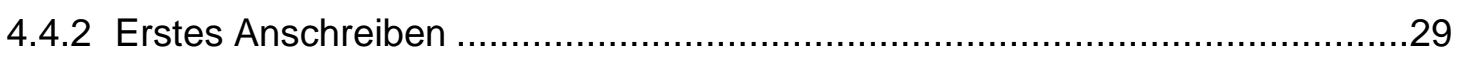

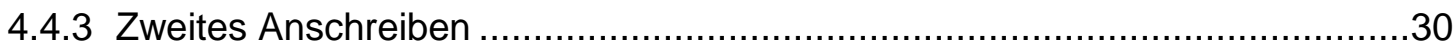

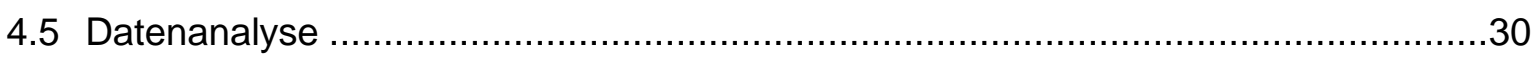

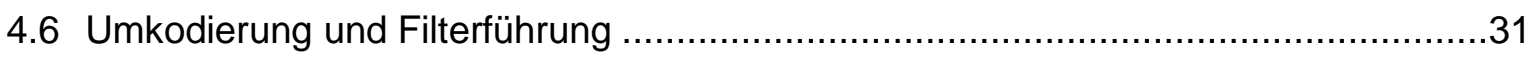

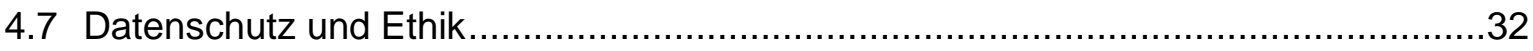

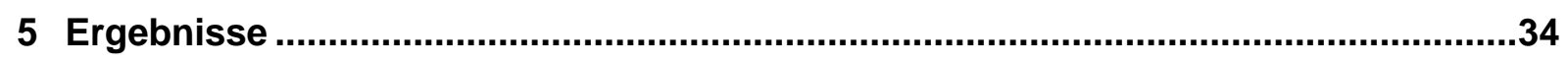

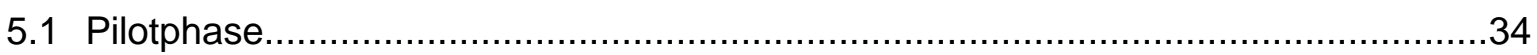

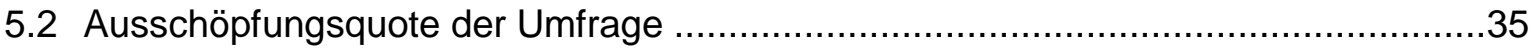

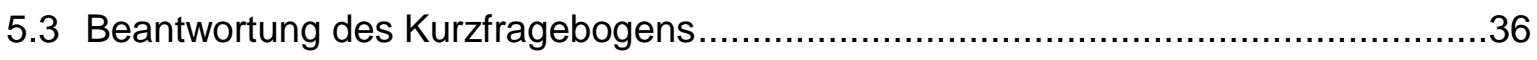

5.4 Beschreibung der Teilnehmer-Stichprobe des Hauptfragebogens ............................36

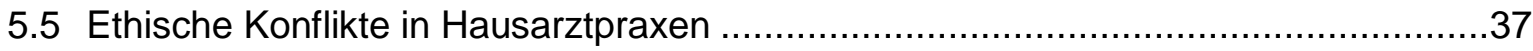

5.6 Teilnahme an Qualitätszirkeln und Balintgruppen und deren Unterstützung in

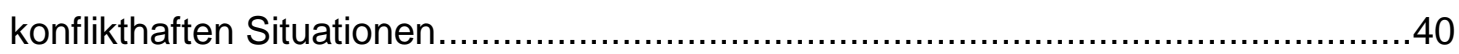

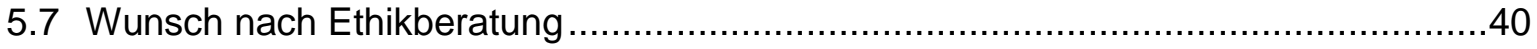

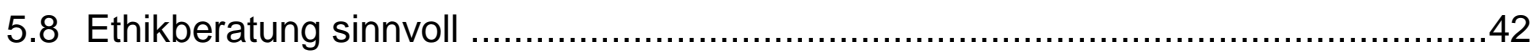

5.9 Angebotswünsche an eine ambulante Ethikberatung …...................................43

5.10 Berufliche Hintergründe und Qualifikationen eines Ethikberaters...........................44

5.11 Vergütung der Teilnahme an einer Ethikberatung ............................................45

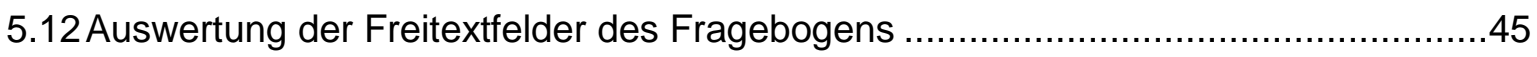

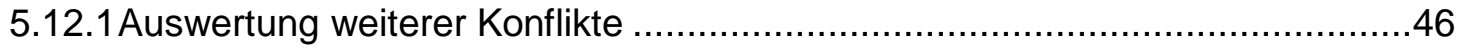

5.12.2Weitere Angebote einer Ethikberatung .............................................. 47

5.12.3Berufliche Hintergründe und Eigenschaften eines Ethikberaters....................47

5.12.4Weitere Qualifikationen eines Ethikberaters .........................................47

5.12.5Zusatzqualifikationen der Teilnehmer .................................................47 


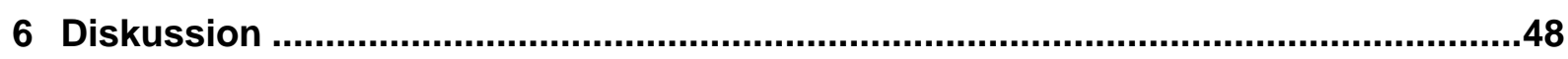

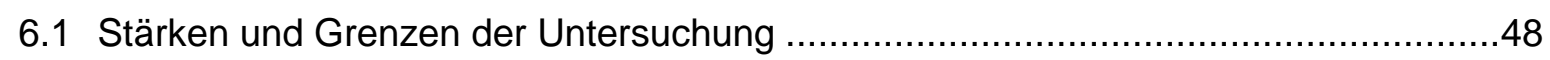

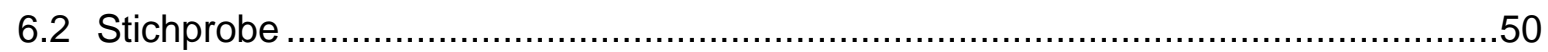

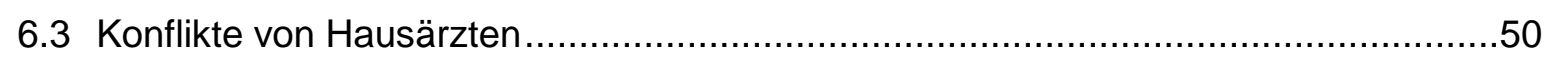

6.4 Teilnahme an Qualitätszirkeln, Supervisions- und Balintgruppen und deren Unterstützung bei der Lösung von Konflikten...................................................58

6.5 Wunsch von Hausärzten nach einer Ethikberatung …........................................58

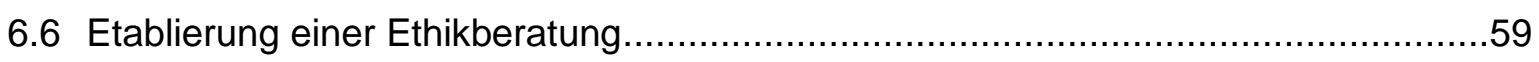

6.7 Angebote, berufliche Hintergründe und Qualifikationen für Ethikberater ...................60

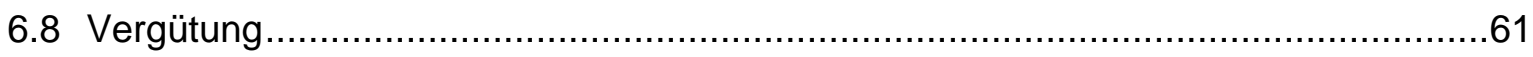

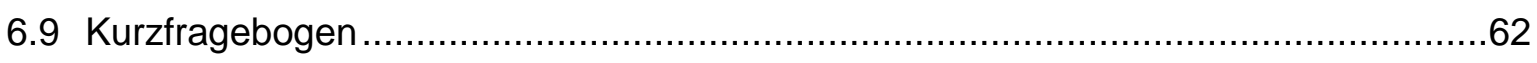

6.10 Praktische Implikationen und weiterer Forschungsbedarf ....................................62

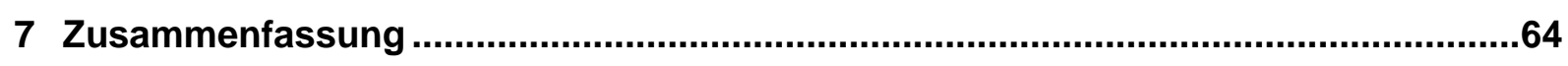

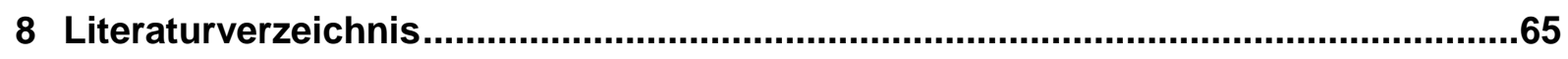

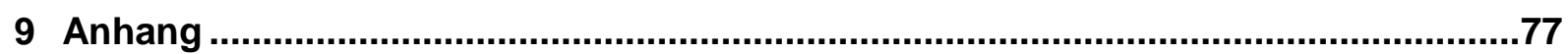

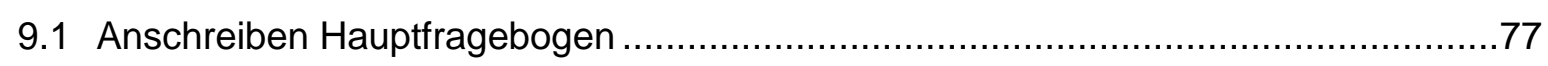

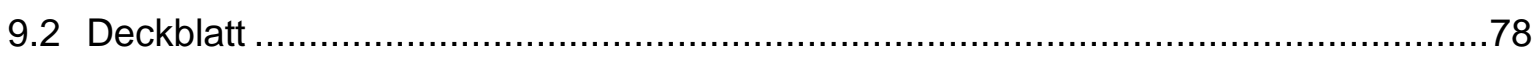

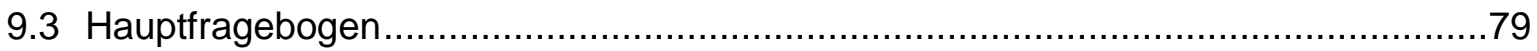

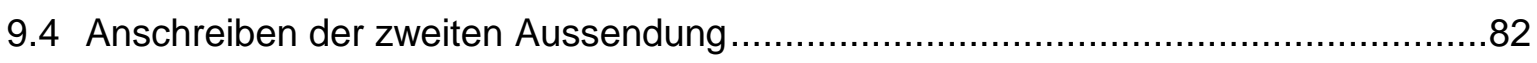

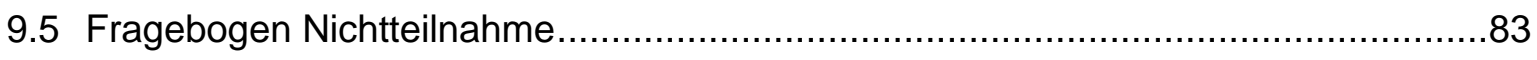

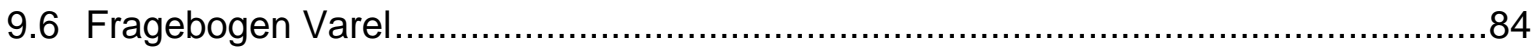

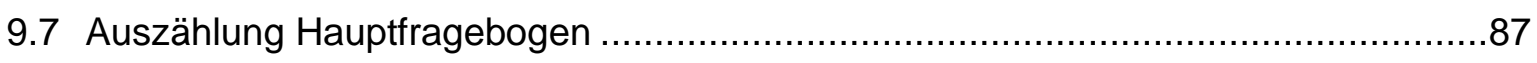

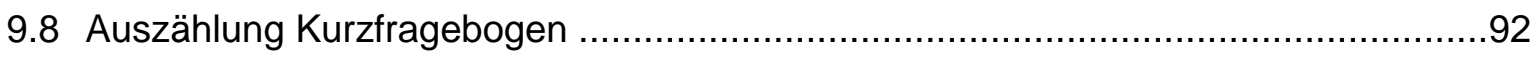

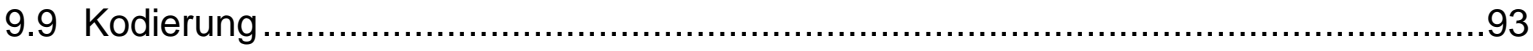




\section{Tabellenverzeichnis}

Tabelle 1: Übersicht über die unterschiedlichen Beratungskonzepte ................................. 3

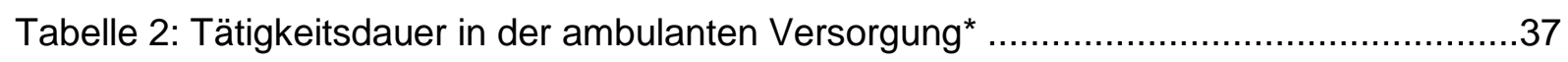

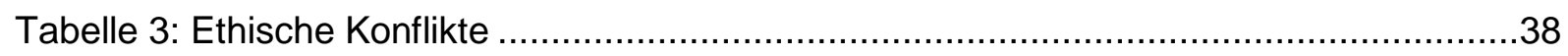

Tabelle 4: Häufigkeit ethischer Konflikte; nach Geschlecht............................................39

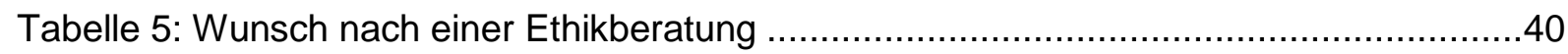

Tabelle 6: Einflussfaktoren im Hinblick auf den Wunsch nach einer Ethikberatung...............41

Tabelle 7: Einflussfaktoren im Hinblick auf die Sinnhaftigkeit einer Ethikberatung ...............42

Tabelle 8: Ethikberatung wird als sinnvoll erachtet ...................................................43

\section{Abbildungsverzeichnis}

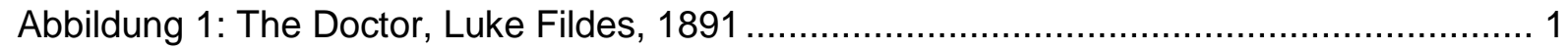

Abbildung 2: Anlaufstellen für eine ambulante Ethikberatung …....................................16

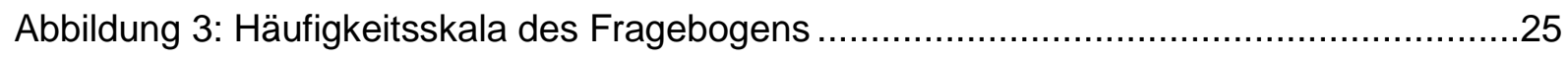

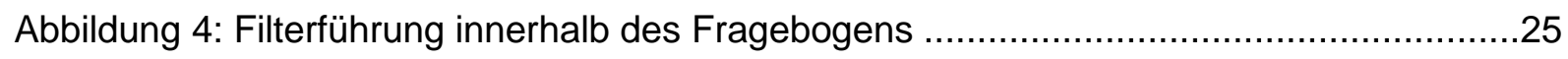

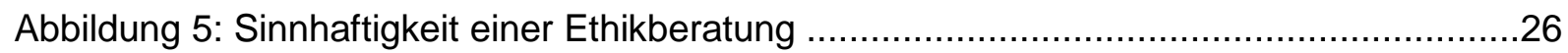

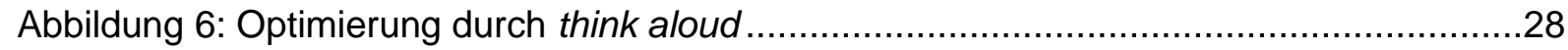

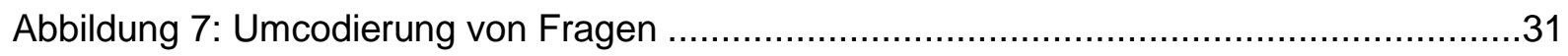

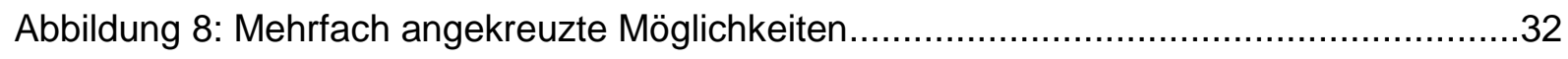

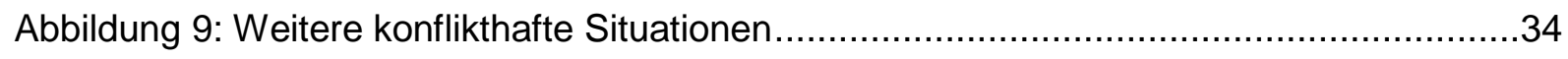

Abbildung 10: Ursprünglicher und veränderter Fragebogen (Hauptumfrage, Frage 1) .........35

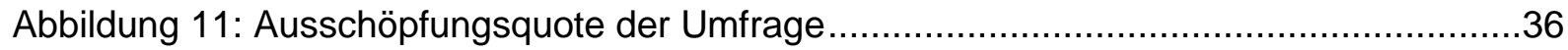

Abbildung 12: Wünsche an eine ambulante Ethikberatung ........................................44 


\section{Einleitung}

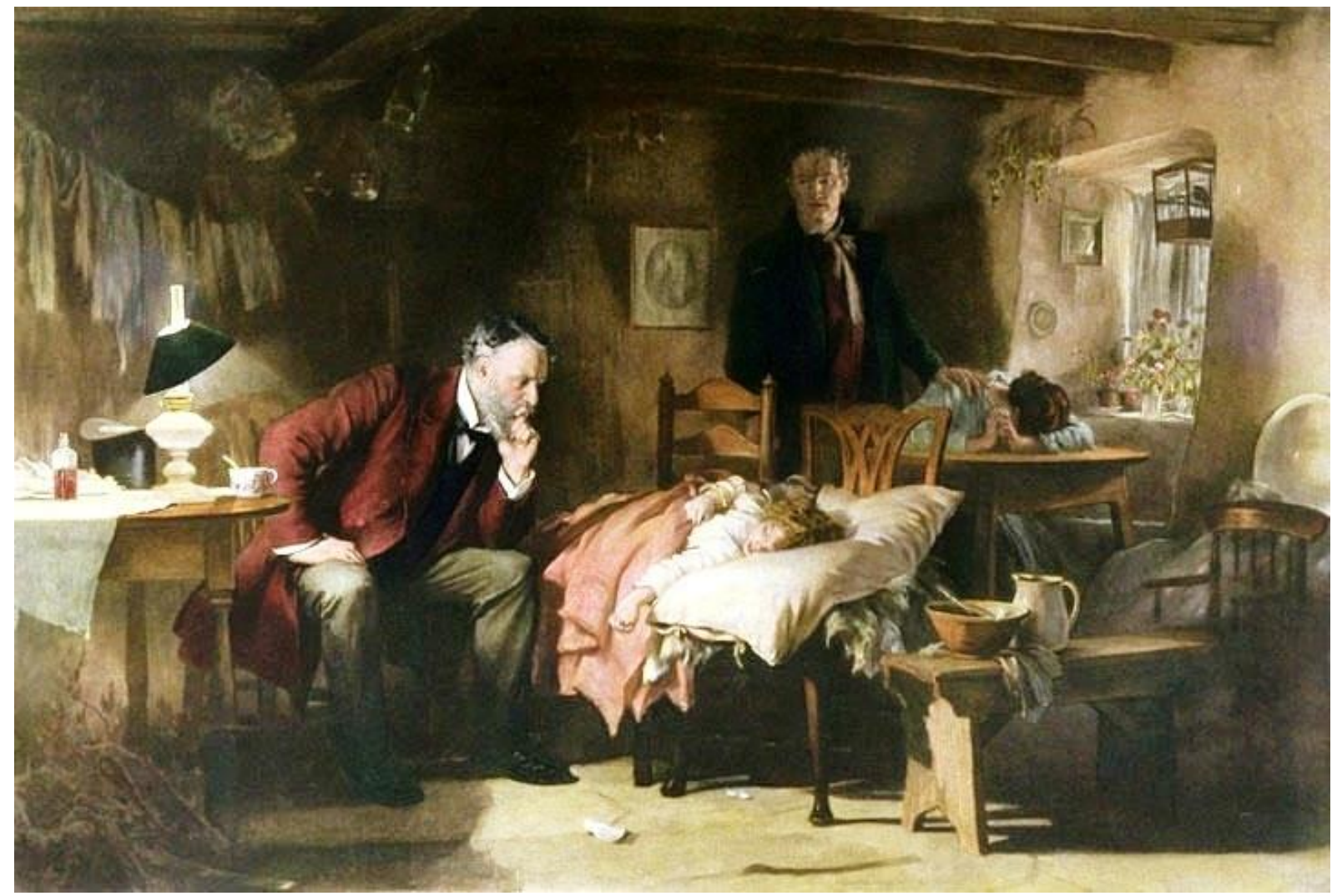

Abbildung 1: The Doctor, Luke Fildes, 1891

Eine 89-jährige, multimorbide, verwitwete Patientin wird mit der Begründung „Allgemeinzustand-Verschlechterung" notfallmäßig ins Krankenhaus gefahren. Zwei Tage später hat sich ihr Zustand nur leicht verbessert, sie wurde mittlerweile auf die Intensivstation verlegt. Dort erzählt sie dem behandelnden Arzt, sie wolle keine lebensverlängernden Maßnahmen mehr über sich ergehen lassen. Sie habe ein schönes, erfülltes Leben gehabt und möchte sterben. Die zwei Kinder der Patientin können den Wunsch ihrer Mutter in keiner Weise nachvollziehen. Sie versuchen ihre Mutter umzustimmen, ein Konsens wird an diesem Tag nicht mehr gefunden. Am nächsten Tag hat sich die Situation der Patienten verschlechtert. Sie ist nicht mehr ansprechbar. Es könne sein, so der behandelnde Arzt zu den herbeigeeilten Angehörigen, dass die Patientin bei einer weiteren Verschlechterung zum Überleben eine Bluttransfusion bräuchte. Die Kinder der alten Dame bitten den Arzt, diese lebensverlängernde Maßnahme anzuordnen, sollte es zu dieser Situation kommen. Einen solchen Schritt lehnte die Patientin am Vortag jedoch ab. Die Beteiligten kommen zu keinem Konsens. Eine involvierte Krankenschwester schlägt eine Ethikberatung vor.

Am darauffolgenden Tag ist der Zustand der Patientin unverändert. In einem ruhigen Zimmer auf der Intensivstation treffen sich der behandelnde Arzt, die Krankenschwester, die zwei 
Kinder der Patientin mit drei Ethikberatern. Gemeinsam überlegen sie, welche Möglichkeiten bestehen und welches Resultat sich die Patientin für sich wünschen würde. Nach einer Dreiviertelstunde intensiver Diskussion und Moderation durch einen der Ethikberater wird ein Konsens gefunden. Die Kinder der Patientin folgen dem letzten Wunsch ihrer Mutter und tragen den Verzicht auf lebensverlängernde Maßnahmen mit.

Anders liegen die Dinge im folgenden Fall: Eine Hausärztin wird von einem Ehepaar aufgesucht. Sie hätten sich schon lange mit dem Thema beschäftigt und wären nun zu einem Konsens gekommen. Beide möchten keine lebensverlängernden Maßnahmen, falls innen etwas zustoßen sollte. Sie bitten die Hausärztin, innen beim Ausfüllen eines Formulars zu helfen, das diesen Wunsch berücksichtigt. Bis auf eine beginnende Demenz der 64-jährigen Ehefrau geht es beiden Patienten gut. Die Hausärztin begleitet beide schon seit Jahren, sie kommt ihrer Bitte nach. Eine Woche später kommt der Ehemann alleine in die Praxis. Aufgelöst erzählt er von starken Schlafstörungen seit letzter Woche. Ein Leben ohne seine Ehefrau wäre für ihn nicht zu ertragen; die Patientenverfügung müsse vernichtet werden. Umstimmen könne er seine Ehefrau nicht, sagt er, und bittet um Hilfe. Die Hausärztin vereinbart einen Termin mit dem Ehepaar in der nächsten Woche. Ein Konsens kann trotz eines langen Gespräches nicht gefunden werden. Die Hausärztin fühlt sich hilflos, eine Entscheidung zu finden fällt ihr schwer.

Medizinische Ethikberatungen haben sich im Alltag von Krankenhäusern mittlerweile etabliert. So helfen professionelle Berater* bei kritischen Situationen zeitnah, die Standpunkte der Beteiligten herauszustellen und, soweit möglich, gemeinsam einen Konsens zu erzielen. In der ambulanten Versorgung findet sich ein derartiges Angebot jedoch kaum. Hausärzte werden mit schwierigen medizinisch-ethischen Entscheidungen häufig alleingelassen; eine professionelle Ethikberatung gibt es selten. Eine Umfrage bei knapp 200 Ärzten aus Bayern ergab, dass es Ärzten an ethischen Kompetenzen mangelt - mit entsprechenden Folgen für die Qualität der medizinischen Betreuung (Wandrowski et al. 2012). Ob sich Hausärzte eine ambulante Ethikberatung wünschen, mit welchen Konflikten sie sich konfrontiert sehen und wie eine solche Beratung aussehen könnte, ist Thema dieser Arbeit.

*Aus Gründen der besseren Lesbarkeit wird in dieser Dissertationsschrift auf die gleichzeitige Verwendung männlicher und weiblicher Sprachformen verzichtet. Sämtliche Personenbezeichnungen gelten - wenn nicht gesondert darauf hingewiesen wird - gleichermaßen für beide Geschlechter. 


\section{Stand der Forschung}

Ethik wird im Angesicht zunehmender technischer Entwicklungen und Ökonomisierungsprozessen ein immer wichtigeres Thema in der Patientenversorgung. In vielen Bereichen der Krankenhausmedizin, aber auch in Hospizen, in der Altenpflege oder in Hausarztpraxen nehmen ethische Konflikte zu (Vollmann 2008a, Bockenheimer-Lucius und May 2007).

Ethikberatungen verstehen sich als Plattform, auf der die Konfliktbeteiligten mit geschulten Ethikberatern Argumente austauschen können, um - wenn möglich - den Konflikt beizulegen. Formen der Ethikberatung lassen sich unter anderem nach medizinischem Kontext und zugrundeliegender Struktur unterscheiden (Tabelle 1).

Tabelle 1: Übersicht über die unterschiedlichen Beratungskonzepte

\begin{tabular}{|c|c|c|c|}
\hline $\begin{array}{l}\text { Formen der } \\
\text { Ethikberatung }\end{array}$ & $\begin{array}{l}\text { Angebundene } \\
\text { Institution }\end{array}$ & Zielperson /-konflikte & Beispiel \\
\hline $\begin{array}{l}\text { Klinische } \\
\text { Ethikberatung }\end{array}$ & Krankenhaus & $\begin{array}{l}\text { Krankenhauspatienten } \\
\text { und ihre Angehörigen }\end{array}$ & $\begin{array}{l}\text { Klinisches } \\
\text { Ethikkomitee }\end{array}$ \\
\hline $\begin{array}{l}\text { Ambulante } \\
\text { Ethikberatung } \\
\text { an/ für bestimmte } \\
\text { Institutionen }\end{array}$ & $\begin{array}{l}\text { Hospiz, Pflegeheim, } \\
\text { Intensivpflege- } \\
\text { abteilung }\end{array}$ & $\begin{array}{l}\text { Heim-/ Hospizbewoh- } \\
\text { ner und Angehörige }\end{array}$ & $\begin{array}{l}\text { Ethikberatung in der } \\
\text { Altenpflege }\end{array}$ \\
\hline $\begin{array}{l}\text { Ambulante } \\
\text { Ethikberatung }\end{array}$ & unterschiedlich & $\begin{array}{l}\text { Patienten und } \\
\text { Angehörige im } \\
\text { ambulanten Bereich }\end{array}$ & $\begin{array}{l}\text { Verschiedene Instituti- } \\
\text { onen, z.B. Netzwerk } \\
\text { ambulante Ethikbera- } \\
\text { tung Göttingen (NEG) } \\
\text { (siehe } 2.7 .1 \text { ) }\end{array}$ \\
\hline
\end{tabular}

Für die weiteren Ausführungen werden im Folgenden wichtige Begriffe der Medizinethik vorgestellt und definiert, um einen einheitlichen Sprachgebrauch im Rahmen dieser Arbeit zu gewährleisten.

Moral. Moral befasst sich mit den Vorstellungen über das Richtige und das Gute, also mit Begriffen, die unser Handeln und unser Zusammenleben praktisch leiten. Im weitesten Sinne bezieht sie sich auf die von einer Gemeinschaft eingelebten Verhaltensnormen (Kruip und Winkler 2007, Hiemetzberger 2010). 
Ethik. Ethik reflektiert die eingeübte Moral einer Gesellschaft. Sie überprüft und begründet Aussagen über das richtige Handeln (Kruip und Winkler 2007). Ethik „versucht zu klären, was moralisch gut oder schlecht ist, richtig oder falsch, geboten oder verboten bzw. gerecht und ungerecht ist" (Hiemetzberger 2010, S. 26).

Ambulante Ethikberatung. Der Aufbau ambulanter Ethikberatungen befindet sich in Deutschland noch in den Anfängen. Derzeit findet sich eine geringe Anzahl an Ethikberatungsangeboten (siehe 2.7.1), diese arbeiten mit heterogenen Konzepten unabhängig voneinander. Eine Richtlinie für den Aufbau ambulanter Ethikberatungen existiert bisher weder in Deutschland noch in anderen Ländern.

Klinische Ethikberatung. Die klinische Ethikberatung „unterstützt ratsuchende Personen (Patienten, Angehörige, Mitarbeiter des Krankenhauses) in einer Konfliktsituation durch Einzelfallberatungen in schwierigen Entscheidungssituationen, sie führt Teamberatungen bei ethischen Konfliktsituationen und Weiterbildung in Fragen der klinischen Ethik durch und erarbeitet Leitlinien für ethische Problemfelder“ (Nauck 2011, S. 138-139). Eine Übertragung dieser Richtlinien, die für Ethikberatungen im Krankenhaus erarbeitet wurden, ist wegen der großen strukturellen und inhaltlichen Richtlinien zwischen dem ambulanten und klinischen Setting nicht möglich (Gágyor 2012).

Klinisches Ethikkomitee. Ethikkomitees bilden das Gremium der klinischen Ethikberatung. Sie sind ein Ansatz zur Verbesserung der Qualität in der Patientenversorgung. Berater entwickeln Leitlinien, organisieren Fort- und Weiterbildung sowie Unterstützung in Einzelfällen anbieten (Akademie für Ethik in der Medizin e. V. 2010). In Deutschland hat sich die klinische Ethikberatung vor allem seit den 1990er Jahren entwickelt (Vollmann 2008a). Sie „trägt dazu bei, schwierige Entscheidungen in der Patientenversorgung auf eine überzeugende, tragfähige Basis von Argumenten zu stellen“ (Simon et al. 2005, S. 325). Die Zusammensetzung eines Ethikkomitees soll das Krankenhaus repräsentieren, Mitglieder kommen meist aus der Medizin, der Pflege, der Theologie, aber auch aus anderen Berufsgruppen (Nauck 2011).

Ethikkommission. Eine Ethikkommission gibt Stellungnahmen zu medizinischen Forschungsvorhaben ab. Sie berät auf gesetzlicher sowie auf berufsrechtlicher Grundlage der medizinischen Hochschulen und darf nicht mit dem klinischen oder ambulanten Ethikkomitee gleichgesetzt werden (Zentrale Ethikkommission 2006).

Hausarzt und hausärztlich tätiger Internist. Hausärzte und hausärztlich tätige Internisten praktizieren in Einzelpraxen, Praxisgemeinschaften, Gemeinschaftspraxen oder neuerdings vermehrt in medizinischen Versorgungszentren. Sie sind für Patienten meist die erste Anlaufstelle im Gesundheitssystem (Slowther 2009), diagnostizieren Krankheiten, überweisen Patienten wenn nötig zu Fachärzten oder in Krankenhäuser und absolvieren Hausbesuche 
(siehe 2.7.2). Der Allgemeinarzt hat den besten Einblick in die psychosoziale Umwelt des Patienten (Abholz und Pillau 2012).

\subsection{Beginn und Entwicklungen der Medizinethik in Amerika}

Die Etablierung von Ethikberatungen an Krankenhäusern schritt in den verschiedenen Ländern unterschiedlich schnell voran (Reiter-Theil 1999). Wenn man den Beginn der klinischen Ethikberatung in Amerika an einem bestimmten Punkt festmachen wollte, wäre dies wohl die Gründung des Ethikkomitees in Seattle, Washington, im Jahr 1962. Es bestand aus sieben Mitgliedern mit unterschiedlichen beruflichen Hintergründen (Jonsen 2007) und wurde aufgrund der Verteilungsschwierigkeiten der wenigen Dialyseplätze ins Leben gerufen. Die Nachfrage nach dieser lebenserhaltenden Maßnahme war größer als das Angebot (Tapper 2013). Das Komitee sollte entscheiden, wer von den schwerkranken Patienten Zugang zu einem Dialyseplatz bekommen sollte. Schnell wurde es als „God-squad“ betitelt, da es manchen Menschen verhalf am Leben zu bleiben, andere aber sterben ließ (Alexander 1962).

Folgende Entwicklungen in der zunehmend technisierten Medizin zwischen 1970 - 1980 verlangten neue Antworten (Tapper 2013):

- Die Erfindung des arterio-venösen Shunts, der Patienten eine ambulante Dialyse ermöglichte.

- Die Verbesserung der Reanimationstechnik, wodurch mehr Patienten irreversibel komatös am Leben gehalten werden konnten, was verschiedene Problematiken mit sich brachte.

- Errungenschaften in der Transplantationsmedizin und die dadurch entstandenen Verteilungsschwierigkeiten.

Vor allem die Entwicklung der verbesserten Reanimationstechnik hatte zur Folge, dass neben der Anzahl an geretteten Leben auch die Zahl der Patienten stieg, die irreversibel komatös am Leben gehalten wurden. Als Folge waren viele Krankenhausbetten belegt, es gab zu wenig Platz für Neuaufnahmen. Neben der Angst, diese komatöse Patienten über einen lange Zeitraum ohne Aussicht auf Besserung zu behandeln, wuchs zusätzlich die Sorge der Ärzte, sich gegen die Reanimation eines Patienten zu entscheiden (Tapper 2013). Auch wenn es gute Gründe gegen eine Wiederbelebung gab, wie eine in der Vergangenheit klar formulierte Ablehnung durch den Patienten oder durch eine eindeutig irreversibel komatöse Situation eines Patienten, fühlten sich Ärzte zur Reanimation verpflichtet. Die Gefahr, sich einer kriminellen Handlung schuldig zu machen, sollten sie nicht reanimieren, war ihnen zu groß. Für Patienten wurde es beinahe unmöglich, ohne Gerichtsurteil zu sterben (Morison 
1981). Es gestaltete sich als schwierig, dieser neuen Entwicklung Regeln aufzuerlegen und sie zu beschränken.

Die Ärzte suchten Antworten auf die Frage, ob es in bestimmten Fällen richtig wär nicht zu reanimieren, welche Kriterien genutzt werden sollten, um einen Patienten für tot zu erklären und wer die Verantwortung für solche Entscheidungen übernehmen sollte. Daraufhin wurde eine Arbeitsgemeinschaft ins Leben gerufen, die eine Definition eines irreversiblen Komas erarbeiten sollte - den Hirntod (Beecher et al. 1968). Bis heute ist diese Definition umstritten und wird kontrovers diskutiert (Müller 2010).

Seit 1992 muss in Amerika jedes Krankenhaus für seine Akkreditierung eine Struktur zum Umgang mit ethischen Konflikten vorweisen (Maio 2002).

\subsection{Beginn und Entwicklungen der Medizinethik in Deutschland}

Die erwähnten Entwicklungen in der Medizin stellten Ärzte auch in anderen Ländern vor neue Herausforderungen. Für die Ethikberatung in Deutschland ist vor allem das Jahr 1997 hervorzuheben. So heißt es in der Stellungsnahme der christlichen Krankenhausverbände aus diesem Jahr:

„Ethische Probleme im Krankenhaus entstehen dann, wenn die allgemeinen Grundsätze ärztlichen und pflegerischen Handelns entweder zu unspezifisch sind, als dass man daraus eine konkrete Handlungsstrategie ableiten kann, oder sie miteinander kollidieren (zum Beispiel Erhaltung des Lebens vs. zu erwartendes qualvolles Dahinsiechen; Leben der Mutter vs. Leben des Kindes bei einer schwierigen Geburt). Ethikkomitees werden sich in einem solchen Fall bemühen, nach bestem Wissen und Gewissen im gemeinsamen Diskurs die relativ beste Lösung zu finden“ (Deutscher evangelischer Krankenhausverband e.V. und Katholischer Krankenhausverband Deutschland e.V. 1997, S. 13).

Eine Umfrage drei Jahre nach dieser Stellungnahme ergab, dass von den ca. 800 konfessionellen Krankenhäusern in Deutschland 30 Krankenhäuser eine klinische Ethikberatung gegründet hatten (Simon 2001). Eine bundesweite Befragung aus dem Jahr 2005 von 2300 Krankenhäusern ergab, dass 312 der 483 (64,6\%) antwortenden Krankenhäuser eine Form einer Ethikberatung anbieten oder sich im Aufbau befinden (Dörries und Hespe-Jungesblut 2007).

Die Stellungnahme der Zentralen Ethikkommission bei der Bundesärztekammer aus dem Jahr 2006 erhöhte ebenfalls die Aufmerksamkeit auf die klinische Ethikberatung. So heißt es:

„Die zentrale Ethikkommission begrüßt die zunehmende Gründung von klinischen Ethikkomitees und anderer klinischer Ethikberatungsstrukturen im deutschen Gesundheitswesen als praxisrelevanten Beitrag zur besseren Versorgung von Patienten und fordert Einrichtungen, in denen derartige Strukturen bisher nicht bestehen, zu ihrer Implementierung auf“ (Zentrale Ethikkommission 2006, S. A1707).

Unterstützt wurde die Etablierung einer Ethikberatung zusätzlich durch eine positive Bewertung bei der Zertifizierung von Krankenhäusern (Simon et al. 2005). 
In den letzten Jahren haben sich klinische Ethikkomitees auch an Universitätskliniken vermehrt entwickelt. Eine Umfrage aus dem Jahr 2008 ergab, dass von den 26 der 36 Universitätskliniken ethische Beratungsstellen oder Klinische Ethikkomitees vorhanden sind und 11 Universitätskliniken dafür Wissenschaftlerstellen geschaffen hatten (Vollmann 2008a).

Im Gegensatz zu den USA ist das Angebot einer Ethikberatung für deutsche Krankenhäuser nicht gesetzlich verpflichtend, wird aber positiv bei Zertifizierungsverfahren der Krankenhäuser berücksichtigt (Simon et al. 2005).

\subsection{Aufgaben und Ziele der klinischen Ethikberatung}

Die neuen technischen Möglichkeiten wie Dialyse, verbesserte Reanimationstechniken und Weiterentwicklungen in der Transplantationsmedizin (siehe 2.1) brachten Verunsicherungen und Zwiespalt innerhalb der Ärzteschaft mit sich. Ziel der klinischen Ethikberatung war, dass Ärzte diese Unsicherheiten gemeinsam mit Experten diskutieren und gemeinsam eine Entscheidung treffen, um potentiell entstehende Schuldgefühle teilen zu können (Tapper 2013). Im Allgemeinen verfolgt die medizinische Ethikberatung drei Ziele (Zentrale Ethikkommission 2006), die im Folgenden näher erläutert werden:

- Beratung bei medizinisch-ethischen Konflikten im Einzelfall

- Entwicklung von ethischen Leitlinien

- Koordination von Fort- und Weiterbildung zu ethischen Themen.

\section{(1) Beratung bei medizinisch-ethischen Konflikten im Einzelfall}

Grundsätzlich wird zwischen Einzelfallberatungen und Gruppenberatungen unterschieden. Einzelfallberatungen können zum Beispiel bei Gewissenskonflikten von einem Beteiligten einberufen werden. An Gruppenberatungen nehmen mehrere Personen teil, möglichst auch der betroffene Patient. Das Gespräch sollte an einem ruhigen Ort stattfinden und von einem Mitglied des abgesandten Gremiums moderiert werden. Dieser Moderator ist dafür verantwortlich, dass alle wichtigen Details zum Fall deutlich werden. Seine Aufgabe ist außerdem „die ethischen Fragen herauszuarbeiten und die Möglichkeiten des weiteren Vorgehens nach ethischen Kriterien zu gewichten“ (Akademie für Ethik in der Medizin e. V. 2010, S. 152). Die Einhaltung des Datenschutzes und der Schweigepflicht müssen gewährleistet sein (Akademie für Ethik in der Medizin e. V. 2010). 


\section{(2) Entwicklung von ethischen Leitlinien}

Leitlinien werden von Mitgliedern des Gremiums entwickelt, gegebenenfalls mit Unterstützung eines Sachkundigen (Akademie für Ethik in der Medizin e. V. 2010). Sollte eine Leitlinie vom Ethikkomitee beschlossen werden, obliegt es der Krankenhausleitung diese verbindlich für die verschiedenen Berufsgruppen zu etablieren (Zentrale Ethikkommission 2006). Leitlinien sollen eine Handlungsempfehlung für wiederkehrende Konflikte wie zum Beispiel das Legen einer PEG-Sonde, Therapiezieländerung oder der Umgang mit Zeugen Jehovas darstellen. Diese Empfehlungen müssen mit dem Gesetz konform sein und regelmäßig aktualisiert werden (Akademie für Ethik in der Medizin e. V. 2010).

\section{(3) Koordination von Fort- und Weiterbildung zu ethischen Themen}

Zielgruppen solcher Fortbildungen sind Angestellte des Gesundheitswesens, Patienten und deren Angehörige und die Öffentlichkeit. Realisiert werden sie durch Mitglieder des Ethikkomitees oder eine andere geeignete Person (Akademie für Ethik in der Medizin e. V. 2010).

Indirekt werden durch die genannten Ziele - neben einer Problemlösung - zusätzlich langfristige Effekte auf die Denk- und Sichtweise der Mitarbeiter erwartet; sie werden für ethische Fragen geschult und reflektieren und kommunizieren über ethische Fragestellungen mit Kollegen - auch professionsübergreifend. Außerdem lernen sie, Erfahrungen und Erkenntnisse in weitere Bereiche der Medizin zu transferieren (Höger et al. 1997).

\subsection{Formen, Ablauf und Organisation klinischer Ethikberatung}

Die Ethikberatung ging in Deutschland zu Beginn von dezentralen Initiativen aus, die sich in verschiedenen Krankenhäusern gebildet hatten. Die fehlende Vernetzung führte zu einem mangelnden Informationsaustausch und einer fehlenden professionellen Begleitung. Somit entstanden unterschiedlichen Konzepte und Modelle der Beratung (Vollmann 2008a). Im Folgenden werden wichtige Beratungsmodelle und Konzepte einer klinischen Ethikberatung sowie der Ablauf einer Beratung skizziert. Zuvor werden der Gründungsprozess und allgemeine Grundsätze einer klinischen Ethikberatung vorgestellt.

\subsubsection{Gründungsprozess}

Vor allem aufgrund einer positiven Bewertung bei Zertifizierungsprozessen bei Nachweis einer Ethikberatung (Simon et al. 2005) geht der Impuls zur Etablierung einer Ethikberatung heutzutage häufig von der Krankenhausleitung aus. Dieses „Top-Down-Modell“ hat den Vorteil, dass von verantwortlicher Stelle Strukturentscheidungen zielstrebig umgesetzt und gefördert werden. Bei dem „Bottom-Up-Modell“ geht die Initiative von Mitarbeitern fern der Leitungsebene aus - mit dem Vorteil einer breiteren Akzeptanz innerhalb der Belegschaft 
(Vollmann 2008a). Für eine erfolgreiche Umsetzung einer Klinischen Ethikberatung sollten beide Bewegungen zueinander finden, denn bei fehlender Unterstützung durch die Krankenhausleitung einerseits sowie bei mangelnder Akzeptanz bei den Krankenhausbeschäftigten andererseits wird sich eine erfolgreiche Umsetzung eines ethischen Beratungsangebotes als schwierig gestalten.

\subsubsection{Allgemeine Grundsätze einer klinischen Ethikberatung}

Jede Form von Ethikberatung wird nur auf Wunsch eines Betroffenen durchgeführt (Vollmann 2008a). Die Moderation einer Ethikberatung sollte durch eine unvoreingenommene, möglichst objektive Person durchgeführt werden, nicht vom Antragssteller oder dem jeweiligen Leiter der Krankenhausstation. Dieser unabhängige Moderator kann am ehesten zur Klärung aller Perspektiven des Patienten beitragen (Reiter-Theil 2000). Außerdem kann dadurch einem potentiellen Machtmissbrauch vorgebeugt werden (Aulisio et al. 2000). Der Moderator verbindet in einer Fallberatung die Technik der Moderation mit ethischer Expertise (May 2011). Das Ergebnis einer Ethikberatung soll eine Unterstützung und keine konkrete Handlungsanweisung darstellen (Vollmann 2008a); die finale Entscheidung trifft der behandelnde Arzt, er trägt auch die rechtliche Verantwortung (Maio 2002).

\subsubsection{Beratungsmodelle und Konzepte der Klinischen Ethikberatung}

Es wird zwischen drei Beratungsmodellen unterschieden, die ihre jeweiligen Vor- und Nachteile haben:

(1) Im Expertenmodell wird der Konflikt von einem Arzt, einem Patienten oder einer weiteren involvierten Person an das klinische Ethikkomitee weitergeleitet. Das Komitee sucht nach einer Lösung, ohne dass die ratsuchenende Person an der Diskussion teilnimmt. Es wird somit nach Aktenlage entschieden (Neitzke 2008). Die Entscheidungssuche findet nicht auf der Krankenhausstation statt, sondern in den Räumen des Ethikkomitees; das Stationsteam ist am Diskussionsprozess nicht beteiligt. Diese Form der Beratung ist für Klinikärzte zeitökonomisch; allerdings sichert es nicht automatisch die Akzeptanz seitens des Stationsteams (Neitzke 2008.)

(2) Im Delegationsmodell wird eine beteiligte Person zum Ethikkomitee entsandt. In den Räumen des Komitees trägt sie dem Gremium den Fall vor und präsentiert die Empfehlung später im Krankenhaus. Vorteilhaft bei diesem Modell ist, dass die delegierte Person dem Komitee mehr Details berichten kann, außerdem kann sie dem Stationsteam anschließend den Entscheidungsprozess erläutern. Bei diesem Modell werden, wie auch beim Expertenmodell, nicht alle beteiligten Personen gehört (Neitzke 2008). 
(3) Im Prozessmodell wird auf Anfrage eine Subgruppe des Ethikkomitees auf die Krankenhausstation entsandt. Der Beratungsprozess ist ein "shared-decision-making“, gemeinsam wird mit möglichst vielen Beteiligten nach einer Lösung gesucht. Dadurch wird die ethische Kompetenz auf der Station gestärkt. Außerdem können mehr Menschen ihre Meinung zum Ausdruck bringen, alle können mitentscheiden, wodurch sich die Verbindlichkeit erhöht (Neitzke 2008).

Das häufigste Konzept ist das des Klinischen Ethikkomitees (Schochow et al. 2014). Dieses Gremium ist angeschlossen an das jeweilige Krankenhaus. Es besteht in der Regel aus sieben bis zwanzig Mitgliedern mit möglichst unterschiedlichen beruflichen Hintergründen und hierarchischen Positionen (Vollmann 2008a). Dadurch vervielfältigen sich die verschiedenen Sichtweisen über die zu behandelnde Problematik, was den Entscheidungsfindungsprozess vorteilhaft beeinflussen kann.

Ein weiteres Konzept ist das des Ethikkonsiliars. Hier stellt ein professioneller Medizinethiker sein Wissen zur Verfügung und moderiert als Außenstehender eine Ethikberatung. Dieser Konsiliar wird als Experte verstanden, der die Entscheidungsfindung aktiv lenkt und steuert (Neitzke 2009). Dieses Modell wird allerdings nur von wenigen Krankenhäusern, hauptsächlich von Universitätskliniken angeboten (Schochow et al. 2014, Gerdes und Richter 1999).

An einigen Krankenhäusern haben sich weitere Modelle wie „Ethik-AGs“, „Ethik Cafés“, „Ethikforen“ etabliert. Diese sind weniger streng organisiert; sie gelten als flexibler, offener und stellen häufig den Ursprung für die Implementierung eines klinischen Ethikkomitees dar (Neitzke 2008). Diese unterschiedlichen Modelle einer klinischen Ethikberatung überschneiden sich teilweise und sind nicht immer klar voneinander abzugrenzen.

Durch die zunehmende Fusion geographisch teilweise weit auseinander liegenden Krankenhäuser zu einem Dachverband findet man auf der übergeordneten Trägerebene zunehmend eine Art Gesamtethikkomitee, welches sich mit ethischen Grundsatzfragen beschäftigt und Fortbildungen organisiert (Vollmann 2008a).

\subsubsection{Ablauf einer klinischen Ethikberatung}

Der Ablauf einer klinischen Ethikberatung wird hier am Beispiel des klinischen Ethikkomitees aufgezeigt; ein entsprechend anderer Ablauf gilt für die beiden anderen Modelle. Die große Mitgliederzahl des Komitees verhindert eine zeitlich und örtlich flexible Beratung (Zentrale Ethikkommission 2006). In der Praxis hat es sich daher bewährt, nach Kontaktaufnahme eine Arbeitsgruppe des Komitees zu entsenden (Vollmann 2008a). Der Entscheidungsfindungsprozess kann somit zeitnah und vor Ort durchgeführt werden. Ein Mitglied der entsendeten Arbeitsgruppe übernimmt die Funktion eines Moderators, er leitet das Gespräch mit dem Behandlungsteam. Ein weiteres Mitglied übernimmt die Funktion des Co-Moderators. 
Der Fall wird mit allen Beteiligten gleichberechtigt diskutiert. Alle Anwesenden unterliegen der Schweigepflicht (Vollmann 2008a).

Es hat sich bewährt, eine ethische Fallbesprechung in sechs Abschnitte zu gliedern (Vollmann 2008c):

Eröffnung. Die Eröffnung beginnt mit der Begrüßung der anwesenden Personen durch den Moderator, Informationen über die Klinische Ethikberatung und einer Vorstellungsrunde der Teilnehmer. Zudem wird die Fragestellung formuliert und ein Zeitrahmen für die Diskussion angesetzt.

Informationen werden zusammengetragen. Die medizinischen, die pflegerischen Fakten und die Sicht des Patienten und der Angehörigen werden zusammengetragen.

Entscheidungskonflikt. Der Entscheidungskonflikt wird konkret benannt. Beispiel: Soll einem älteren Patienten nach einem Schlaganfall mit schweren Schluckstörungen eine Ernährungssonde gelegt werden?

Benennung der ethischen Fragestellung. Auf dieser Ebene wird die spezielle ethische Problematik des vorliegenden Entscheidungskonfliktes formuliert. In Bezug auf das obige Beispiel werden Argumente anhand ethischer Prinzipen für und gegen das Legen einer Ernährungssonde gesammelt.

Diskussion der ethischen Fragen. Die gesammelten ethischen Argumente werden in Bezug auf den vorliegenden Fall diskutiert.

Abschluss. Das Ergebnis der Diskussion wird festgehalten; wenn ein Konsens erreicht wurde, wird dieser als Empfehlung formuliert. Den Teilnehmern wird eine weitere Beratung angeboten. Im Anschluss gibt es die Möglichkeit einer Rückmeldung an den Moderator.

\subsection{Anforderungen an einen klinischen Ethikberater}

Aufgrund der ethisch sehr heterogenen Situationen, die in einem Krankenhaus entstehen können, sind die Anforderungen an die Fähigkeiten eines klinischen Ethikberaters vielfältig. $\mathrm{Ob}$ es spezieller Qualifikationen und Ausbildungen für Ethikberater bedarf, war lange Zeit umstritten (Tapper 2013). Laut Aleksandrova 2008 sollen Ethikberater entweder ausgebildete Ethiker mit klinischer Erfahrung sein oder erfahrene Kliniker mit einer zusätzlichen ethischen Ausbildung. Aulisio et al. 2000 vertreten dagegen die Meinung, dass es keine spezielle Zertifizierung für Ethikberater geben sollte. Sie sollen allerdings über folgende Kernkompetenzen verfügen: Wissen (knowledge), Fähigkeiten (skills) und verschiedene Charakterzüge (character traits). Das anzueignende Wissen teilt sich in moralische Argumentation und ethische Theorien, bioethische Konzepte sowie die Kenntnis über Richtlinien und Grundsätze des eigenen Krankenhauses und spezielle rechtliche Aspekte der Medizin. Als wichtige Fä- 
higkeit sollten Mitglieder klinischer Ethikberatungen zwischenmenschliche Kompetenzen und Fähigkeiten zu Prozessbeurteilung aufweisen können sowie die Fähigkeit, Probleme ethisch beurteilen können. Zu den zu erwerbenden Charakterzügen zählen Ehrlichkeit, Offenheit, Toleranz, Zivilcourage, Integrität und Menschlichkeit. Diese Kompetenzen sollten durch die Gesamtheit des Teams vereint werden, nicht jedes Mitglied des Komitees muss über alle Anforderungen verfügen (Aulisio et al. 2000).

Nach Reiter-Theil 1999, S. 228 sollten Ethikberater über (1) „professionelle Kompetenz und Erfahrung im Umgang mit Patienten“, (2) über „fundierte Kenntnisse in Ethik“ und (3) „spezifische medizinethische Kompetenzen“ verfügen sowie (4) „Training und Erfahrung in Gesprächsführung“ aufweisen. Außerdem werde eine gewisse (5) „Persönlichkeitsbildung“ vorausgesetzt. Ethikberater sollten Mitgliedern jeder Abteilung die Möglichkeit geben, ihre Sicht der Dinge darzulegen (Steinkamp und Gordijn 2001), auch die des Patienten sollte artikuliert werden (Craig und May 2006). Sie sollten zudem in der Lage sein, mögliche Diskrepanzen zwischen Patientenerwartung und Realität zu schließen und grundsätzlich die Entscheidungsfindung optimieren (Craig und May 2006).

Erwähnenswert ist in diesem Zusammenhang eine Befragung aus dem Jahr 2004 an 112 bulgarischen Ärztinnen und Ärzten zum Thema Kompetenzen eines Ethikberaters. Hauptwunsch war, dass der Berater über „klinische Kompetenz“ verfügt (71\%), gefolgt von der „Möglichkeit, mit Konflikten umzugehen“ (59\%). Er soll „Kommunikationsfähigkeiten“ (58\%) besitzen, „Toleranz für verschiedene Sichtweisen“ (55\%) aufbringen und „speziellen Qualifikationen in Ethik“ (53\%) aufweisen (Aleksandrova 2008, S. 40).

\subsection{Sichtweisen und Nutzung klinischer Ethikberatung durch medizinisches Fachpersonal}

Trotz der anfänglichen Schwierigkeiten und Vorbehalte entwickelte sich die klinische Ethikberatung kontinuierlich weiter. In den USA findet Ethikberatung mittlerweile in $81 \%$ aller Krankenhäuser statt und in 100\% der Krankenhäuser mit über 400 Betten (Fox et al. 2007).

Eine Studie aus Norwegen bewertet die Arbeit medizinischer Ethikkomitees als hilfreich (Kalager et al. 2011). Zu einem ähnlichen Ergebnis kommt eine amerikanische Studie: Über $70 \%$ der befragten Ärzte sagten, sie hätten durch die Beratung etwas für die Zukunft gelernt (DuVal et al. 2004); 90\% der befragten Teilnehmer einer weiteren Studie unterstützten die Aussage, Ethikberatung spiele eine wichtige Rolle, um ethische Sachverhalte zu identifizieren und zu analysieren; 74\% bestätigten die Aussage, Ethikberatung spiele eine wichtige Rolle, um das medizinische Team auszubilden und über $90 \%$ würden Ethikberatung weiterempfehlen (Yen und Schneiderman 1999). In der gerade erwähnten Befragung der bulgari- 
schen Ärzte empfanden knapp 90\% der Befragten eine Ethikberatung als vorteilhaft, $81 \%$ würden eine Ethikberatung anfordern (Aleksandrova 2008).

Eine amerikanische Studie untersuchte die Patienten, für die eine Ethikberatung einberufen wurde. Demnach waren sie in der Mehrzahl der Fälle männlich und zwischen 50 bis 69 Jahren alt; fast die Hälfte der Patienten lag auf einer Intensivstation (Wasson et al. 2015).

\subsubsection{Gründe für die Inanspruchnahme einer klinischen Ethikberatung}

Der medizinische Fortschritt, aber auch steigende Gesundheitskosten, erhöhte Erwartungen und Ansprüche der Patienten, ein genereller Wertewandel in der Gesellschaft und Ressourcenknappheit sind für die zunehmend komplexer werdenden medizinischen Fragestellungen und deren Beantwortung verantwortlich (Aleksandrova 2008). Häufig entstehen Konflikte „zwischen hochrangigen Prinzipen im Vordergrund“ (Leben schützen vs. sterben lassen) (Reiter-Theil 1999, S. 226, Kalager et al. 2011, Wasson et al. 2015). Weitere Konfliktfelder betreffen die Patientenautonomie, Konflikte zwischen involvierten Personen, Substanzmissbrauch, religiöse und kulturelle Themen und die Frage nach Abtreibungen. Ob dem Patienten die Wahrheit zugemutet werden kann sowie rechtliche Fragen (DuVal et al. 2001, Kalager et al. 2011, Wasson et al. 2015), Entscheidungen über Therapieabbruch bzw. Sinnlosigkeit medizinischer Behandlungen (Reiter-Theil 2000) und ökonomische Rahmenbedingungen (Vollmann 2008b) sind zusätzliche Konfliktfelder.

Anlässe für Ethikberatungen lassen sich somit klassifizieren in:

- Konflikte über die Fortsetzung einer Therapie am Lebensende

- Unsicherheit über den Willen oder das Wohl des Patienten

- Uneinigkeit der beteiligten Parteien

- Schwierigkeiten mit unkooperativen Patienten (nach Reiter-Theil 1999, S. 226).

In der Untersuchung der bulgarischen Ärzteschaft empfanden 83 der 112 (77\%) befragten Ärzte die Beziehung mit Patienten und Angehören als erlebtes ethisches Dilemma, 34 Teilnehmer (32\%) empfanden Konflikte am Lebensende („End-of-life issues“) und 31 Ärzte (29\%) Ressourcenknappheit als erlebtes Spannungsfeld (Aleksandrova 2008).

\subsubsection{Gründe gegen die Inanspruchnahme einer klinischen Ethikberatung}

Ein Problem klinischer Ethikberatung besteht darin, dass sie Forderungen nach einer objektiven Evaluation häufig nicht gerecht werden kann. Parameter, die für die Evaluation anderer Verfahren genutzt werden, sind bei der Bewertung von Ethikberatung unzureichend (Craig und May 2006). Da sich die individuellen Werte der Patienten häufig voneinander unter- 
scheiden, differieren auch die Ziele der Beratungen. Eine Evaluation ist daher nur schwer möglich (Craig und May 2006). Gegner einer Ethikberatung kritisieren, Beratungen wären zu zeitintensiv, wären nicht hilfreich und Berater würden Sachlagen nur noch verschlimmern. Außerdem wären Lösungen der Berater oft nicht mit guter Medizin in Einklang zu bringen (DuVal et al. 2004). Kritiker sehen in Ethikkomitees eine Zusammenballung unkontrollierbarer Entscheidungsmacht, wodurch die Gefahr eine Rückkehr zur paternalistischen Medizin bestehe. Handlungsempfehlungen des Ethikkomitees bekämen einen allgemeinen Geltungsanspruch, was zu einer Verdünnung oder Auflösung von individueller moralischer Verantwortung in bürokratische Strukturen führen würde (Kettner und May 2004). Die Klinische Ethikberatung sei „weder wissenschaftlich belegt und akzeptabel noch in der Praxis umsetzbar“ (Strätling und Sedemund-Adib 2013, S. A825). Ungeklärt sei zudem die Qualität von „faktisch meist selbst ernannten Ethikberatern“ (Strätling und Sedemund-Adib 2013, S. A826). Es würden nur wenige Beratungen im Jahr stattfinden und die Komitees beständen überwiegend aus fachfremden Mitgliedern, vor allem aus Vertretern der christlichen Kirche, die auch nicht-medizinische Ziele verfolgen. Die Delegierung der Medizinethik habe sich als ernst zunehmende Fehlentwicklung erwiesen (Strätling und Sedemund-Adib 2013). Insgesamt seien klinische Ethikkomitees weit hinter den Erwartungen zurückgeblieben und weder von Ärzten noch von Pflegenden, Juristen oder Patienten akzeptiert (Strätling und Sedemund-Adib 2013).

\subsubsection{Gegenwärtige klinische Ethikberatung in Deutschland}

Eine vorhandene Ethikberatung wird positiv bei Zertifizierungsverfahren im Rahmen von Qualitätssicherung von Institutionen im Gesundheitssystem gewertet (Vollmann 2008a). Eine Umfrage an die jeweiligen Klinikdirektoren von 1858 Krankenhäusern in Deutschland ergab, dass etwa $75 \%$ der 529 teilnehmenden Krankenhäuser die Struktur für eine klinische Ethikberatung vorhalten (Schochow et al. 2014). Dabei steigt die Implementierungsquote mit der Größe der Krankenhäuser: kleine Krankenhäuser (bis 300 Betten) besitzen in etwa 65\% der Fälle eine Ethikberatung, mittlere Krankenhäuser (301-700 Betten) zu etwa 82\% und große Krankenhäuser (ab 700 Betten) zu fast 90\%. Die Implementierungsquote ist unabhängig von der Trägerschaft des Krankenhauses, konfessionsgebundene Krankenhäuser führen allerdings häufiger Ethikberatungen durch (Schochow et al. 2014). Teilnehmer an Beratungen sind in den meistern Fällen Ärzte, Theologen, Seelsorger und Pflegedienstmitarbeiter. Wenige Krankenhäuser $(22,1 \%)$ stellen der Ethikberatung ein Budget zur Verfügung. Fast $10 \%$ der Befragten stimmten der Aussage voll zu, dass durch klinische Ethikberatung Probleme und Grenzsituationen umfassend gelöst wurden, 62,1\% stimmten eher zu, 19,1\% stimmten eher nicht zu, 4,0\% stimmten gar nicht zu (Schochow et al. 2014); Ethikberatungen scheinen 
damit für viele Teilnehmer ein geeignetes Medium zur Lösung von Konflikten in Krankenhäusern zu sein.

\subsection{Ethikberatung in der ambulanten Gesundheitsversorgung}

Ethikberatung entwickelte sich besonders dort, wo es häufig zu medizinischen Extremsituationen kommt, überwiegend also in der Intensivmedizin (Slowther 2009). Eine Ethikberatung konzentriert sich somit vor allem auf Konflikte und Fragestellungen im Krankenhaus. Ethische Fragestellungen entstehen aber auch in anderen Institutionen, wodurch Ethikberatungsangebote auch außerhalb von Krankenhäusern zunehmend an Bedeutung gewinnen, zum Beispiel in Altenpflegeheimen und Hospizen (Bockenheimer-Lucius und May 2007). Häufig sind Palliativnetzwerke die Initiatoren für die Gründung ambulanter Ethikberatungen, da sich in der Versorgung von Palliativpatienten immer wieder ethische Fragen stellen, die mehrere Institutionen des Gesundheitssystems betreffen (Altenpflegeheim, Hausärzte, Hospize etc.). Außerdem sind Palliativnetzwerke ein Gefüge, an das die Ethikberatung angeschlossen werden kann (Simon 2015).

Beratungsangebote speziell für Hausärzte sind weiterhin eine Seltenheit, obwohl auch in diesem Bereich zunehmend Alltagsprobleme aufgrund der medizinischen Möglichkeiten und der juristischen Unsicherheiten auftreten. Auf dem 111. Ärztetag im Mai 2008 in Ulm wurde diese Entwicklung zum Anlass genommen, eine „... geeignete, aber auch berufsrechtskonforme Maßnahme für eine ambulante Ethikberatung in Deutschland zu entwickeln, um Hausärzte bei ethischen Grenzfällen eine Unterstützung zur Einholung einer fachlichen Zweitmeinung oder eines Ethikvotums anbieten zu können“ (Bundesärztekammer 2008, S. 32). Drei Aufgaben einer ambulanten Ethikberatung wurden formuliert:

- Beratung bei medizinischen und ethischen Konflikten im Einzelfall

- Veröffentlichung von ethischen Entscheidungen im Ärzteblatt

- Koordination von Fort- und Weiterbildung zu ethischen Themen. 


\subsubsection{Angebote einer Ethikberatung für Allgemeinärzte}

In den letzten Jahren nahmen die Angebote für Ethikberatungen im ambulanten Bereich zu (siehe Abbildung 2). Betrachtet man die Konzepte der verschiedenen Institutionen, ergibt sich dabei ein deutlich heterogenes Bild. Eine spezielle Ethikberatung für Hausärzte bieten bisher nur wenige Einrichtungen, obwohl vor allem Hausärzte in die Versorgung von Patienten am Lebensende involviert sind (Gágyor et al. 2013, Gágyor et al. 2016). Eine internationale Studie kam zu dem Ergebnis, dass nur 17,6\% von 656 befragten Ärzte aus vier europäischen Ländern (Großbritannien, Norwegen, Italien, Schweiz) Zugang zu einer Ethikberatung bei individuellen Problemen haben (Hurst et al. 2008). Einbezogen waren Ärzte, die Zugang zu Krankenhäusern hatten oder Patienten stationär betreuten. Die tatsächliche Unterstützung für ambulant tätige Ärzte ist somit wahrscheinlich noch weit geringer (Slowther 2009).

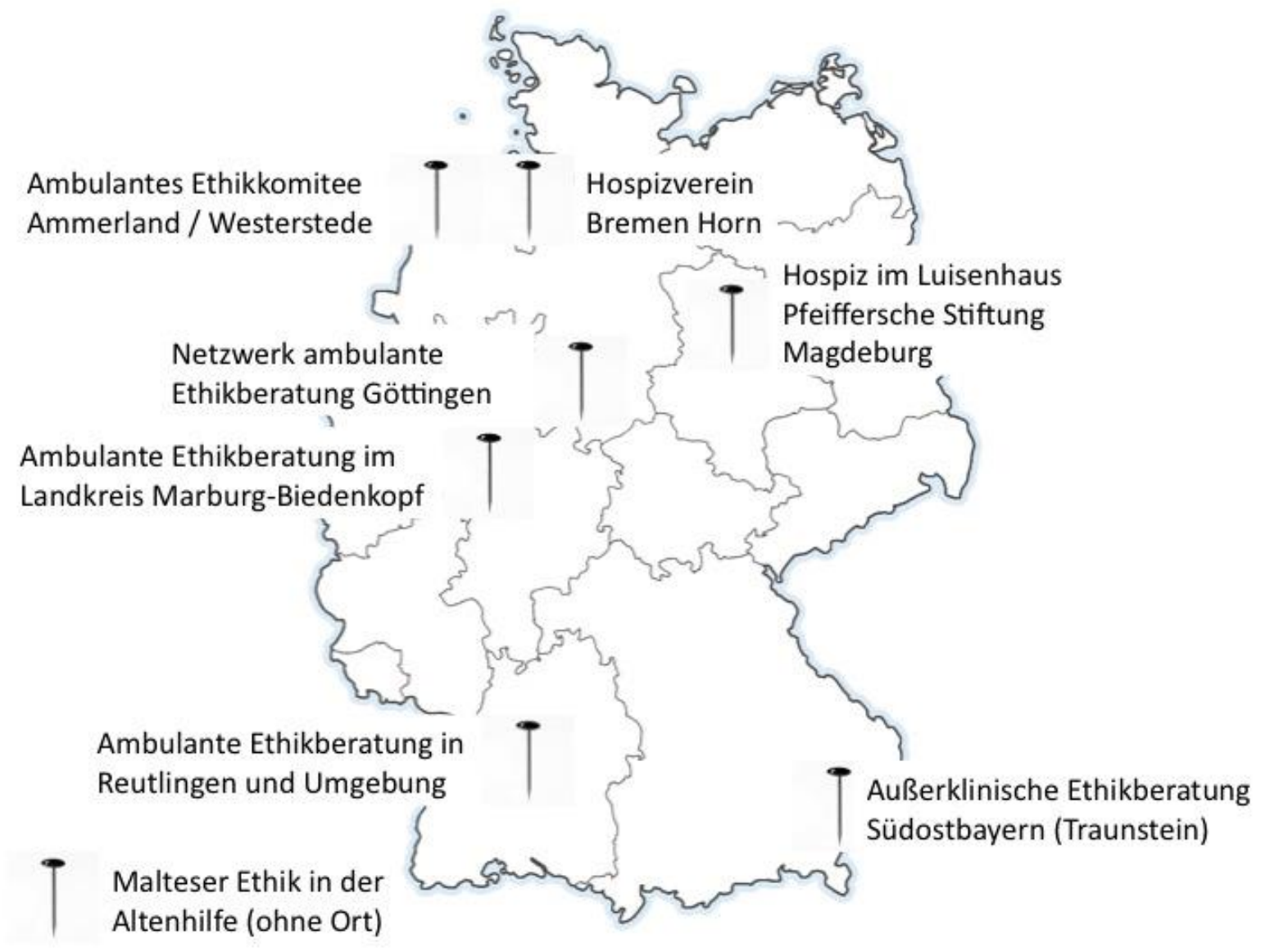

Abbildung 2: Anlaufstellen für eine ambulante Ethikberatung

Im Folgenden werden zur Veranschaulichung einige Beispiele bestehender ambulanter Ethikberatungen vorgestellt:

Netzwerk ambulante Ethikberatung Göttingen (NEG). Die Klinik für Palliativmedizin an der Universität Göttingen bietet seit einiger Zeit eine spezialisierte ambulante Patientenversorgung (SAPV) für hausärztliche Ethikberatungen an. Das Ethikkomitee besteht aus ca. 35 
ehrenamtlichen Mitgliedern, unter anderem Pflegekräfte, Hausärzte und Seelsorger. Die Beratungen können telefonisch, am Patientenbett, im Pflegeheim oder an einem neutralen Ort stattfinden und sind kostenlos (Allgemeinmedizin Göttingen 2015). Dieses Angebot wird einbis zweimal pro Monat genutzt (Gágyor 2012).

Außerklinische Ethikberatung Südostbayern (Traunstein). Das Team in Traunstein besteht aus Ärzten, Pflegenden, Seelsorgern und Anwälten sowie einem Notar. Die Kosten der Beratung werden vom Netzwerk Hospiz übernommen. Eine Ethikberatung können neben Hausärzten und anderen Fachärzten unter anderem auch Patienten und Angehörige, Betreuungsrichter, Brückenpflegekräfte, Seelsorger erfragen. Die ärztliche Hoheit verbleibt bei dem behandelnden Hausarzt, seine Teilnahme an der Ethikberatung ist verpflichtend (Netzwerk Hospiz 2012).

Ambulantes Ethikkomitee Ammerland/ Uplengen. Dieses Ethikkomitee wurde im Sommer 2013 der Öffentlichkeit vorgestellt. Mitglieder sind unter anderem Altenpfleger, Apotheker, Hausärzte, Fachärzte für Neurologie, Erzieher, Juristen, Krankenpfleger und Seelsorger. Sie wurden in einer 80 Stunden umfassenden Ausbildung zu Moderatoren ethischer Fallbesprechungen ausgebildet, finanziell wurde diese Ausbildung vom Förderverein des Palliativstützpunktes getragen. Anfragen an eine individuelle Fallberatung können alle Bewohner der Region stellen. Sie wird dem Vorstand des ambulanten Ethikkomitees vorgelegt; dieser entscheidet, ob es sich um eine ethische Fragestellung handelt und beauftragt gegebenenfalls drei Mitglieder mit der Fallbearbeitung (Kreft et al. 2015).

\subsubsection{Spezifika der allgemeinärztlichen Praxis im Unterschied zum Haus der Maximalversorgung}

Die folgenden Charakteristika der Allgemeinmedizin gelten zum Teil auch für andere ärztlichen Disziplinen; sie spielen aber vor allem in der Allgemeinmedizin eine bedeutsame Rolle und zeigen die spezifische Bedeutung einer Ethikberatung in der Allgemeinmedizin.

Setting. Hausärzte arbeiten im Regelfall in ihren eigenen Praxen. Dort haben sie „einen weitaus größeren Handlungsspielraum, denn ihnen obliegt gleichzeitig die Leitung und Organisation der Praxis sowie die unmittelbare Arbeit mit den Patienten und deren Angehörigen“ (Gágyor 2012, S. 144). Für die Entscheidungsfindung bei Konflikten steht im ambulanten Bereich mehr Zeit zur Verfügung (Kreft et al. 2015).

Die Arbeit von Hausärzten im Behandlungszimmer oder bei Hausbesuchen in der Wohnung der Patienten ist relativ isoliert. Entscheidungen des Arztes und das scheinbare Einverständnis des Patienten können dazu führen, dass der Patient mit der Entscheidung nur aufgrund der isolierten Situation konform geht (Slowther 2009). 
Die Arbeit von Hausärzten und im Krankenhaus tätigen Ärzten unterscheidet sich in ökonomischen Gesichtspunkten. Während klinisch praktizierende Ärzte in den wenigsten Fällen direkt für die Finanzen des Krankenhauses verantwortlich sind, gestaltet sich die Selbstständigkeit eines niedergelassenen Allgemeinarztes finanziell risikoreicher.

Patientenkontakt. Hausärzte haben meist den ersten Kontakt zum Patienten. Sie sind es, die entscheiden müssen, ob sie einen Patienten in ein Krankenhaus oder zu anderen Fachärzten überweisen (Slowther 2009).

Das Wissen über den Patienten weist Unterschiede zwischen Allgemeinärzten und Klinikern auf. Im Gegensatz zu im Krankenhaus praktizierenden Ärzten kennen Allgemeinärzte ihre Patienten häufig seit mehreren Jahren und haben deshalb meist einen guten Kenntnisstand über die Krankengeschichte und das soziale Umfeld ihres Patienten (Gágyor 2012). Ihnen sind familiäre Konflikte, Partner- und Erziehungsprobleme, drohende Arbeitslosigkeit oder auch finanzielle Überforderungen häufig bekannt (Abholz und Pillau 2012). Ethische Konflikte können dadurch anders wahrgenommen werden, als es im klinischen Bereich der Fall ist.

Team. Allgemeinärzte arbeiten, im Gegensatz zu im Krankenhaus tätigen Ärzten, in einem kleinen Team mit wenigen Kollegen oder Angestellten; ein Abstimmen mit anderen Ärzten bei problematischen Behandlungsfragen wird somit erschwert. Ärzte im Krankenhaus können sich jederzeit mit ärztlichen Kollegen oder dem Pflegeteam besprechen und abstimmen. Der Allgemeinarzt ist auch bei Hausbesuchen selten zeitgleich mit dem Pflegeteam vor Ort. Eine Absprache gestaltet sich somit mühsam und zeitintensiv.

Zu behandelnde Krankheiten. Im Gegensatz zu Krankenhäusern und anderen Facharztrichtungen werden Hausärzte mit unselektierten Patientenproblemen einer großen Spannweite konfrontiert. Diese Vielfalt reicht von gutartigen Bagatellerkrankungen bis hin zu Frühstadien lebensbedrohlicher Erkrankungen (Donner-Banzhoff 2008). Krankenhausärzte oder andere Fachärzte sind dagegen Spezialisten für einen begrenzten Bereich (Slowther 2009).

Die Allgemeinmedizinpraxen sind technisch anders ausgestattet als Krankenhäuser (DonnerBanzhoff 2008), Entscheidungen über Leben und Tod stellen sich hier weniger häufig oder anders (Slowther 2009). Medizinische Extremsituationen und hohe technische Eingriffe sind eher im Krankenhaus üblich; Ärzte müssen dort nicht selten über Leben und Tod eines Patienten entscheiden.

Hausarztpraxen sind häufiger als Krankenhäuser soziale Anlaufstellen (Slowther 2009). Sie müssen somit neben physischen Erkrankungen auch psychische Leiden behandeln. 
Ältere Patienten haben häufig verschiedene chronische Erkrankungen gleichzeitig, die Behandlung der einen Erkrankung kann eine Kontraindikation für die andere sein - ein optimales Outcome für alle Erkrankungen ist selten zu erreichen (Abholz 2008).

Die Allgemeinmedizin arbeitet im sogenannten Niedrig-Prävalenzbereich, was bedeutet, dass Hausärzte mit selten vorkommenden Krankheiten auch selten konfrontiert werden (Abholz 2008). Diagnostisch sollen in der Allgemeinmedizin nicht immer alle Krankheiten, welche die Symptome potentiell hervorrufen könnten, getestet werden. Die falsch-positiven Befunde würden die richtig-positiven überschreiten - mit erheblichen Konsequenzen für die Patienten (Abholz 2008).

Familienkonstellation. Hausärzte betreuen häufig mehrere Mitglieder einer Familie, wodurch Interessenskonflikte entstehen können (Slowther 2009, Karazivan und Pless 2011).

Krankheiten und Sorgen von Patienten können durch das Verhalten anderer Familienmitglieder begründet sein. So kann sich eine Mutter große Sorgen um ihre Tochter machen, der es in letzter Zeit offensichtlich schlechtgeht. Der Grund dafür kann in einer ungewollten Schwangerschaft der Tochter liegen, die den Arzt darüber in Kenntnis setzt, nicht aber die eigene Mutter.

Familienmitglieder können voneinander in unterschiedlichen Arten abhängig sein. Entscheidet sich der Hausarzt im Zweifelsfall für die Einweisung eines Familienmitglieds ins Krankenhaus, kann das weitreichende Konsequenzen für Familienmitglieder des Patienten nach sich ziehen. Auch bei divergierenden Wünschen zwischen Patient und Angehörigen muss er sich positionieren und macht sich dadurch angreifbar.

Weitere Spezifika und Konfliktfelder der Allgemeinmedizin. Diagnostische und rechtliche Unsicherheiten (Slowther 2009), die Beziehung zu Kollegen (Braunack-Mayer 2001), Behandlungsablehnung (Connelly und DalleMura 1988) und Ressourcenverwendung (Ayres 1996) sind weitere Konfliktfelder in der Allgemeinmedizin.

Auch das Verhältnis zu im Krankenhaus tätigen Ärzten kann konflikthaft sein: Hausärzte werden häufig aufgrund ihrer Einweisungen von im Krankenhaus tätigen Ärzten beurteilt (Slowther 2009). Das knappe Bettenkontingent der Krankenhäuser kann Hausärzten dabei zusätzliche Schwierigkeiten bereiten (Hurst et al. 2006). Eine ambulante Ethikberatung könnte möglicherweise unterstützend tätig werden. 


\subsubsection{Schwierigkeiten einer Etablierung der Ethikberatung für Hausärzte}

Im Krankenhaus kann ein Beratungsangebot räumlich angebunden werden. Dies gestaltet sich aufgrund der geographisch weit verteilten Hausarztpraxen im Hinblick auf die Etablierung einer Ethikberatung für Allgemeinmediziner als schwieriger (Slowther 2009). Ein weiteres Problemfeld ist die Finanzierung einer Ethikberatung, wenn diese nicht an einer speziellen Institution angebunden ist (Slowther 2009). Der Mangel an zuständigen Institutionen und die fehlende akademische Unterstützung ist eine weitere Barriere.

Ein Grund für die bisher wenigen Angebote und Entwicklungen einer Ethikberatung in der Allgemeinmedizin sind neben den in 2.7.2 aufgeführten Spezifika auch im Bereich der Aufgaben und Behandlungsbilder der Hausärzte zu suchen. Diese entscheiden weniger häufig über Leben und Tod eines Patienten, wodurch die Allgemeinmedizin das öffentliche Interesse, den öffentliche Druck und auch die Aufmerksamkeit der Gerichte weniger auf sich zieht. Probleme, die eine Ethikberatung erforderlich machen würden, werden somit weniger registriert (Slowther 2009).

\subsection{Zusammenfassung}

Ethikberatung im Krankenhaus mit ihren verschiedenen Modellen und Konzepten hat sich mittlerweile in vielen Ländern, auch in Deutschland, etabliert und bewährt. In der ambulanten Patientenversorgung fehlt eine solche Beratung weitgehend, ihre strukturelle Verankerung wird nun aber auch für den ambulanten Bereich gefordert (Bundesärztekammer 2008). Ein ethischer Konflikt kann den behandelnden Arzt, auch wenn er selten auftritt, stark belasten. Auch ein geringer Beratungsbedarf seltener als einmal pro Monat, wie es in Göttingen der Fall ist, würde Beratungsangebot rechtfertigen (Gágyor 2012).

Es kann aufgrund der Forderung des 111. Ärztetages angenommen werden, dass sich ein Großteil der Allgemeinärzte eine Ethikberatung wünscht. Auf einen Beratungsbedarf in der hausärztlichen Patientenversorgung deuten auch die bisherigen Beratungsangebote hin (Gágyor 2012). Die Datenlage hierzu ist bisher allerdings spärlich. Sowohl Art und Häufigkeit ethischer Konflikte in der hausärztlichen Versorgung als auch Vorstellungen und Wünsche von Hausärzten an eine ambulante Ethikberatung sind bisher nicht untersucht worden.

Es kann davon ausgegangen werden, dass Konflikte aus dem klinischen Altag nicht mit denen des ambulanten Bereiches übereinstimmen. Erkenntnisse aus dem Bereich der stationären Ethikberatung lassen sich daher nur bedingt für die ambulante Ethikberatung übernehmen. Wünschenswert wäre es, mittels einer Befragung einer großen Zahl von Hausärzten die Häufigkeit medizinisch-ethisch relevanter Konfliktfälle zu untersuchen, um den hausärztlichen Bedarf nach Unterstützung festzustellen und das Informationsdefizit bezüglich der Konflikte von Hausärzten schließen zu können. 


\section{Fragestellung und Ziel}

Mit Hilfe einer Befragung soll die Häufigkeit ethischer Konflikte, mit der sich Hausärzte im ambulanten Bereich konfrontiert sehen, erhoben werden. Zudem soll die Frage beantwortet werden, ob Hausärzte eine ambulante Ethikberatung als sinnvoll erachten und welche Wünsche sie an eine solche Einrichtung haben. Auf diese Weise soll der Bedarf an einer ambulanten Ethikberatung festgestellt werden, worauf er sich begründet und wie entsprechende Angebote strukturiert sein sollten.

Schwerpunkte der Befragung sind:

- aktuelle Konflikte und deren Häufigkeit

- Teilnahme an bereits bestehenden Unterstützungsangeboten

- Bewertung und Einschätzung einer ambulanten Ethikberatung

- Gewünschte Zusammensetzung eines ambulanten Ethikkomitees, Kompetenzen der Mitarbeiter und Erreichbarkeit.

Forschungsleitend waren folgende Hypothesen:

1) Einige ethische Konflikte in Hausarztpraxen sind vergleichsweise häufig, z.B. das Umsetzen einer Patientenverfügung, wenn dieser beispielsweise in einer Notfallsituation nicht nachgekommen wurde.

2) Bereits bestehende Unterstützungsangebote helfen bei der Lösung von Konflikten.

3) Mehr junge als ältere Hausärzte wünschen sich eine Ethikberatung und empfinden deren Etablierung als sinnvoll.

4) Hausärzte in einer Einzelpraxis wünschen sich häufiger eine Ethikberatung

Die gewonnenen Erkenntnisse können als Grundlage für eine mögliche zukünftige Etablierung einer Ethikberatung dienen. 


\section{Methode}

Thema der Arbeit sind ethische Konflikte in der hausärztlichen Tätigkeit und Wünsche an eine mögliche ambulante Ethikberatung. Hierzu wurden Hausärzte (Fachärzte für Allgemeinmedizin, Praktische Ärzte, Fachärzte für Innere Medizin - hausärztliche Versorgung) befragt, soweit sie in Einzelpraxen, Gemeinschaftspraxen, Praxisgemeinschaften und medizinischen Versorgungszentren (MVZ) tätig sind.

\subsection{Methodische Vorüberlegung}

Um einen möglichst großen Adressatenkreis zu erreichen und damit repräsentative Aussagen zu erhalten, schien ein postalisch zu versendender Fragebogen als Methode der quantitativen empirischen Sozialforschung besonders geeignet. Diese Methode gilt als unaufdringlich und anonym (Leiblein und Oglesby 1992). Bewährte Empfehlungen aus der Literatur wurden für das Anschreiben (siehe Anlage 9.1) und den Fragebogen (siehe Anlage 9.3) berücksichtigt.

\subsubsection{Das Anschreiben}

Ein attraktives Anschreiben mit Bezug zum Thema hat sich in der Praxis bewährt (Porst 1998) und sollte sich u.a. durch folgende Bestandteile auszeichnen (Klein und Porst 2000, Menold 2014):

- ein seriöser Briefkopf

- klare Benennung des Themas der Umfrage

- Nennung der durchführenden Institution

- Erläuterung der Zielsetzung der Studie

- persönliche Anrede

- höfliche Aufforderung zur Teilnahme

- Informationen über den Durchführenden

- Zusicherung der Anonymität

- Informationen zur Rücksendung

- Name und Telefonnummer eines Ansprechpartners

- persönliche Unterzeichnung. 
Folgende Maßnahmen können zusätzlich zur Erhöhung der Teilnahmebereitschaft führen (Menold 2014):

- über die Umfrage informieren

- um Unterstützung bitten

- Nutzen der Umfrage aufzeigen

- für die Teilnahme danken

- Vertraulichkeit im Umgang mit Daten und Datenschutz garantieren.

\subsubsection{Der Hauptfragebogen}

Der Fragebogen sollte übersichtlich, klar gegliedert und gut verständlich sein, um eine möglichst schnelle und unkomplizierte Beantwortung zu gewährleisten und dadurch eine hohe Rücklaufrate mit einer guten Antwortqualität zu erhalten. Auch hierzu wurden einschlägige Empfehlungen aus der Literatur beachtet:

- Von großer Bedeutung für die Kooperationsbereitschaft des Befragten ist der erste Eindruck des Fragebogens (Schnell et al. 1998). Der Fragebogen sollte „attraktiv gestaltet, übersichtlich gedruckt und gut lesbar sein“ (Porst 1998, S. 34 - 35). Aus diesem Grund wurde sowohl das Anschreiben als auch der Fragebogen möglichst benutzerfreundlich gestaltet, zum Beispiel indem die Fragen in roter Farbe gedruckt wurden.

- Die Fragen sind so anzuordnen, dass sie für den Adressaten eine flüssige Beantwortung zulassen und den logischen Gedankenfluss durch einen strukturierten Aufbau gewährleisten (Atteslander 2000).

- Um die Logik des Befragungsablaufes gut nachvollziehen zu können, sollten gleiche Themen zu einem Frageblock zusammengefasst werden (Porst 1998).

- „Einstiegsfragen sollten so konstruiert sein, dass sie von allen Befragten zu beantworten sind“ (Porst 1998, S.32). Einstiegsfragen sollen einerseits leicht genug sein, um den Befragten auf die Thematik einzustimmen (Menold 2014), andererseits aber auch interessant sein, da dadurch die Ausschöpfungsquote bei schriftlichen Befragungen gesteigert werden kann (Klein und Porst 2000).

- Demographische Fragen sollten nicht am Anfang des Fragebogens stehen (Menold 2014), da diese sonst „alle Aufmerksamkeit auf sich ziehen und die eigentliche, dann erst folgende Thematik“ unwichtig erscheint (Klein und Porst 2000, S. 29). 
Für die Formulierung der Fragen selbst wurden die „10 Gebote“ von Porst 2014 berücksichtigt:

- Du sollst einfache, unzweideutige Begriffe verwenden, die von allen Befragten in gleicher Weise verstanden werden!

- Du sollst lange und komplexe Fragen vermeiden!

- Du sollst hypothetische Fragen vermeiden!

- Du sollst doppelte Stimuli und Verneinungen vermeiden!

- Du sollst Unterstellungen und suggestive Fragen vermeiden!

- Du sollst Fragen vermeiden, die auf Informationen abzielen, über die viele Befragte mutmaßlich nicht verfügen!

- Du sollst Fragen mit eindeutigem zeitlichen Bezug verwenden!

- Du sollst Antwortkategorien verwenden, die erschöpfend und disjunkt (überschneidungsfrei) sind!

- Du sollst sicherstellen, dass der Kontext einer Frage sich nicht auf deren Beantwortung auswirkt!

- Du sollst unklare Begriffe definieren!

\subsubsection{Der Kurzfragebogen}

Anzunehmen ist, dass vor allem Ärzte antworten, die Interesse an einer ambulanten Ethikberatung haben. Um auch eine Antwort von Ärzten zu erhalten, die nicht an einer ambulanten Ethikberatung interessiert sind, wurde dem zweiten Anschreiben ein Kurzfragebogen beigelegt. Auf dem Kurzfragebogen konnten die Ärzte ankreuzen, aus welchem Grund sie nicht an der Hauptumfrage teilnehmen. Besonders interessant bei der Auswertung des Kurzfragebogens war, wie häufig die Antwortmöglichkeit „... da ich eine ambulante Ethikberatung nicht nutzen würde" angekreuzt wurde.

\subsection{Aufbau des Hauptfragebogens}

Für die Studie wurden insgesamt 17 Fragen erarbeitet, im Umfang von drei Druckseiten, in vier Teile gegliedert (siehe Anlage 9.3). Es gab Single-Choice, Multiple-Choice und offene Fragen. 


\section{Teil 1 - Häufigkeit ethischer Konflikte}

Ethische Konflikte für Hausärzte wurden bereits durch das Institut für Allgemeinmedizin Göttingen qualitativ exploriert (Heßling et al. 2014). Die dabei gefundenen und näher beschriebenen Konflikte sollten nun quantitativ untersucht wurden, um das Themenspektrum, mit dem sich ambulante Ethikberater gegebenenfalls auseinandersetzen müssen, und die erforderliche Qualifikation einschätzen zu können. Insgesamt wurden den Teilnehmern 12 Konflikte präsentiert. Sie wurden aufgefordert, die Situationen anhand einer Rang-Skala nach Häufigkeit zu beurteilen (siehe Abbildung 3). Anschließend hatten die Ärzte die Möglichkeit, eigene Konflikte zu benennen, um gegebenenfalls weitere persönlich belastende Situationen mitteilen zu können.

\begin{tabular}{|c|c|c|c|c|c|}
\hline \multicolumn{6}{|c|}{$\begin{array}{l}\text { Wie oft sind Sie in Ihrer hausärztlichen Tätigkeit mit folgenden } \\
\text { Entscheidungskonflikten konfrontiert? }\end{array}$} \\
\hline & $\begin{array}{l}\text { ca. 1-mal } \\
\text { im } \\
\text { Monat }\end{array}$ & $\begin{array}{l}\text { ca.1-mal } \\
\text { alle } \\
3 \text { Monate }\end{array}$ & $\begin{array}{l}\text { ca. } 1-\text { mal } \\
\text { alle } \\
6 \text { Monate }\end{array}$ & $\begin{array}{l}\text { seltener } \\
\text { als alle } \\
6 \text { Monate }\end{array}$ & $\begin{array}{l}\text { bisher } \\
\text { nicht } \\
\text { erlebt }\end{array}$ \\
\hline $\begin{array}{l}\text { Konträre Behandlungsansich- } \\
\text { ten zwischen mir und einer } \\
\text { stationären Einrichtung. }\end{array}$ & $\square_{5}$ & $\square_{4}$ & $\square_{3}$ & $\square_{2}$ & $\square_{1}$ \\
\hline
\end{tabular}

Abbildung 3: Häufigkeitsskala des Fragebogens

\section{Teil 2 - Bisherige Nutzung von Unterstützungsangeboten}

Der zweite Teil fragte nach der Nutzung bereits bestehender Unterstützungsangebote, zum Beispiel Balintgruppen, Supervisionsgruppen oder Qualitätszirkeln, die im Folgenden näher beschrieben werden. Die Hausärzte wurden gefragt, ob sie diese Möglichkeiten als Unterstützung sehen. Das folgende Beispiel zeigt auch die im Fragebogen verwendete Filterführung (bei „nein“ in Frage 3: Sprung zu Frage 5, siehe Abbildung 4).

Nehmen Sie an Qualitätszirkeln teil?
$\square_{1} \mathrm{ja}$
$\square_{0}$ nein

[falls nein, weiter mit Frage 5]

Haben Sie in Qualitätszirkeln Unterstützung in konflikthaften Situationen erhalten?
$\square_{1} \mathrm{ja}$
$\square_{2}$ war bisher nicht nötig
$\square_{0}$ nein

Abbildung 4: Filterführung innerhalb des Fragebogens 
Zum besseren Verständnis werden im Folgenden Qualitätszirkel und Balintgruppen definiert. Die Aufgabe von Qualitätszirkeln ist unter anderem „eine hohe Qualität ärztlichen Handelns in der Aus- und Weiterbildung zu schaffen und zu erhalten“" (Kassenärztliche Bundesvereinigung 2010, S. 2). Die Teilnehmer tauschen sich über verschiedene Themen regelmäßig aus, um eine hohe Qualität der eigenen Arbeit zu garantieren. Dies geschieht unter anderem durch den Austausch über „Reflexion und Weiterentwicklung der eigenen Tätigkeit“ (Kassenärztliche Bundesvereinigung 2010, S. 4), „Feststellen der Übereinstimmung mit evidenzbasierten Leitlinien“ oder der „Entwicklung von Handlungsempfehlung für die Praxis“ (Kassenärztliche Bundesvereinigung 2010, S. 4).

Balintgruppen wurden Ende der 1940er Jahre von Michael Balint mit dem Ziel entwickelt, den Umgang mit Übertragungs- und Gegenübertragungsphänomenen zwischen Arzt und Patient zu erkennen und damit zu arbeiten. Teilnehmer sollen „Beziehungsdiagnostik und deren Umsetzbarkeit in der altäglichen Praxis“ lernen (Rappe-Giesecke 2000, S. 37). Acht bis zwölf Teilnehmer treffen sich dazu unter der Leitung eines ausgebildeten Balintgruppenleiters und diskutieren Fälle aus dem Praxisalltag. Seit einigen Jahren ist eine wachsende Tendenz zu beobachten, diese Methode über den ärztlichen Berufsstand hinaus für andere Berufsgruppen zu etablieren (Burger 2008). Aufgrund der Veränderungen, die Balintgruppen in den Jahren erfahren haben, schlägt Kornelia Rappe-Giesecke folgende Definition einer Balintgruppe vor:

„Balintgruppenarbeit ist eine Form von Gruppensupervision, in der Angehörige einer Profession, die beruflich und privat unabhängig voneinander sind, sich mit Hilfe eines/einer psychoanalytisch psychotherapeutisch gebildeten Leiters/Leiterin mit der Analyse von Professional-Klient-Interaktionen befassen. Ziel ist die Erhöhung der professionellen Kompetenz im Umgang mit KlientenInnen und der bewußte Umgang mit der professionellen Rolle. Voraussetzung dafür ist eine an die professionelle Rolle und den jeweiligen Fall gebundene Selbsterfahrung" (Rappe-Giesecke 1994, S. 5).

\section{Teil 3 - Fragen zur Ethikberatung, mögliche Mitglieder und deren Kompetenzen}

Im Kern dieses Teils ging es darum, ob die Teilnehmer die Etablierung einer ambulanten Ethikberatung als sinnvoll erachten (siehe Abbildung 5) und welche Erreichbarkeit sie wünschten. Weiterhin wurde erfragt, welche Berufsgruppen Mitglieder des Gremiums sein sollten und über welche Kompetenzen diese verfügen sollten. Den Abschluss dieses Teils bildete die Frage nach der Vergütung des Hausarztes, sollte er eine Ethikberatung in Anspruch nehmen.

Halten Sie die Etablierung einer ambulanten Ethikberatung für sinnvoll?
$\square_{1}$ ja
$\square_{2}$ bin mir nicht sicher
$\square_{0}$ nein

[falls nein, weiter mit Frage 13]

Abbildung 5: Sinnhaftigkeit einer Ethikberatung 


\section{Teil 4 - Demographie}

Zuletzt wurden die Teilnehmer um Informationen zu Geschlecht, Alter, Zusatzqualifikation, Beginn der Patientenversorgung und Praxisart gebeten.

\subsection{Optimierung des Fragebogens}

Vor Beginn der Hauptbefragung wurde der Fragebogen durch verschiedene Methoden geprüft, um mögliche Fehlerquellen und Missverständnisse zu beseitigen und gegebenenfalls neue Ideen in den Fragebogen zu integrieren.

\subsubsection{Probing-Phase}

Die erste Phase zur Überprüfung des Fragebogens bestand aus einem Treffen mit Mitarbeitern des Instituts für Allgemeinmedizin in Göttingen im August 2014. Die Arbeitsgruppe bestand aus einem Soziologen, einer Arzthelferin, zwei Krankenschwestern und dem Doktoranden. Die Teilnehmer sollten in erster Linie sprachliche Missverständnisse aufdecken und die Fragen und Antworten auf Verständlichkeit überprüfen. Außerdem sollte das Design des Fragebogens beurteilt und, wenn nötig, modifiziert werden.

Um den Frage-Antwort-Prozess zu untersuchen, wurde die kognitive Technik think aloud angewendet. Hierfür stellten sich zwei Hausärzten im November 2014 zur Verfügung. Bei dieser Technik wird der Teilnehmer aufgefordert „laut zu denken“, während er seine Antwort formuliert (Porst 1998). Diese Technik „funktioniert am besten bei retrospektiven Fragen“ (Prüfer und Rexroth 2005, S. 14).

Ziel ist es zu untersuchen, wie die Teilnehmer

- „Fragen oder Begriffe interpretieren und verstehen,

- Informationen und Ereignisse aus dem Gedächtnis abrufen,

- Entscheidungen darüber treffen, wie sie antworten,

- ihre ,intern' ermittelte Antwort formalen Antwortkategorien zuordnen“ (Prüfer und Rexroth 2005, S. 3).

Im Anschluss an das think aloud wurde der Fragebogen den beiden Ärzten vorgelegt. Die Aufgabe war es, den Fragebogen auf Fehler und missverständliche Formulierungen zu prüfen und die Sinnhaftigkeit der Fragen zu bewerten. Das Gespräch wurde aufgezeichnet und analysiert, der Fragebogen wurde optimiert. 
Würden Sie eine ambulante Ethikberatung eher in Anspruch nehmen, wenn die aufgewendete Zeit vergütet würde?

$\square_{1} \mathrm{ja}$

$\square_{0}$ nein

Abbildung 6: Optimierung durch think aloud

Ein Arzt antwortete auf diese Frage mit den Worten „Weiß nicht.“ Diese Möglichkeit konnte bis dahin nicht angegeben werden. Der Fragebogen wurde mit dem Item „bin mir nicht sicher" erweitert (siehe Anlage 9.3).

\subsubsection{Pilotstudie}

"If you don't have the resources to pilot test your questionnaire, don't do the study."

(Bradburn et al. 2004, S. 317)

Der Fragebogen wurde im November 2014 an einer Stichprobe von neun Hausärzten aus der Stadt Varel pilotiert, die zu diesem Zeitpunkt die hausärztliche Versorgung der Stadt leisteten. Varel als Stadt mit etwa 25.000 Einwohnern in Niedersachsen schien geeignet, da

- aus der Pilotstichprobe eine Antwortquote abgeschätzt werden sollte. In Varel praktizierten neun Hausärzte, eine Datenerhebung war damit problemlos möglich.

- die angeschriebenen Ärzte in der Hauptbefragung nicht erneut angeschrieben werden sollten - es fielen nur wenige Ärzte weg.

- die Universitätsstadt Göttingen als ,überforscht' gilt.

Das versendete Material unterschied sich bis auf den Fragebogen nicht von dem der Hauptumfrage (siehe 4.3.2), dieser wurde nach der Auswertung der Pilotstudie optimiert (siehe unten). Es sollte untersucht werden, welche Schwierigkeiten und Engpässe sich bei der Befragung ergeben könnten und mit welcher Response gerechnet werden kann. Die Antwortquote sollte als Anhaltspunkt dienen, wie viele Hausärzte bei der Hauptbefragung angeschrieben werden, um der Umfrage die nötige Aussagekraft zu geben. Außerdem sollten etwaige technische Probleme identifiziert werden, um die Organisation der Umfrage zu optimieren.

Die Antworten wurden analysiert und der Fragebogen verändert. So wurde zum Beispiel das Layout verändert und handschriftlich ergänzte Konflikte der Ärzte in den Fragebogen integriert, außerdem wurden die Häufigkeitsangaben bezüglich der erlebten Konflikte modifiziert.

Die Ärzte, die nicht geantwortet hatten, wurden telefonisch nach dem Grund ihrer Nichtteilnahme befragt, um weitere mögliche Fehlerquellen ausschließen zu können. 


\subsection{Durchführung der Hauptbefragung}

Die Datenerhebung fand von Dezember 2014 bis März 2015 statt. Die Ärzte, die an der ersten Umfrage nicht teilnahmen, wurden ein zweites Mal angeschrieben. Beide Anschreiben wurden an einem Mittwoch versendet, damit die Fragebögen möglichst an einem Freitag in den Praxen ankommen konnten. Der Fragebogen sollte den Hausarzt am Ende der Woche erreichen, da zu Beginn der Woche vermutlich mehr Menschen in ärztlichen Wartezimmern sitzen (Deutsche Gesundheitsnachrichten 2012).

\subsubsection{Stichprobenschätzung}

Für die Bestimmung der Stichprobengröße der Hausärzte wurde eine Frage nach der Häufigkeit von ethischen Konflikten ausgewählt, die im Zentrum des Forschungsinteresses steht. Für die späteren Maßnahmen wäre es insbesondere wichtig zu wissen, wie viele Teilnehmer mindestens einmal im Vierteljahr (= Antwortkategorien häufiger als 1 Mal im Monat, ca. $1 \mathrm{Mal}$ im Monat, ca. 1 Mal im Vierteljahr) den folgenden ethischen Konflikt wahrnehmen: „Nachträgliches Umsetzen einer Patientenverfügung, wenn dieser beispielsweise in einer Notfallsituation nicht nachgekommen wurde“. Aufgrund von Vorerfahrungen wurde davon ausgegangen, dass ca. 70 Prozent der Teilnehmer so antworten könnten. Niedersachsen verfügte zum Zeitpunkt der Befragung über ca. 4000 Hausärzte. Um deren Antwort auf die Frage in einem Vertrauensintervall von max. 5 Prozentpunkten Breite absichern zu können, wurden 322 Teilnehmer benötigt. Es wurde- nach Vorerfahrungen - von einer Response in Höhe von ca. 40 Prozent ausgegangen. Daraus ergab sich ein Adressaten-Kreis von $n=800$ Hausärzten. Ein Zufallsprogramm wählte aus einer Datei, in der alle in Niedersachsen zugelassenen Hausärzte aufgeführt sind, 1000 Ärzte aus, um die Wahrscheinlichkeit zu erhöhen, genügend Antwortbögen zu bekommen.

Die im Zuge der Pilotphase angeschriebenen Ärzte aus Varel wurden vorher aus der Datei entfernt. Aus unterschiedlichen Gründen konnten sieben Briefe nicht zugestellt werden, als Ausgleich wurden sieben bisher nicht kontaktierte Hausärzte angeschrieben.

\subsubsection{Erstes Anschreiben}

Das erste Anschreiben fand am 04.12.2014 statt. Es wurde folgendes Material versendet: $\underline{\text { Informationsschreiben } 1}$

Das Anschreiben (siehe Anlage 9.1) informierte die Hausärzte über das Ziel der Studie. Explizit wurden Ärzte gebeten, den Fragebogen auch dann auszufüllen, wenn sie nicht an der Errichtung einer Ethikberatung interessiert sind. Dadurch sollte die Response-Rate erhöht 
werden, außerdem sollten auch die Ärzte motiviert werden zu antworten, die einer Etablierung kritisch gegenüberstehen.

\section{Deckblatt}

Auf dem Deckblatt (siehe Anlage 9.2) war die Adresse des angeschriebenen Arztes notiert. Es wurde an den Fragebogen geheftet, so dass bei Rücksendung des Fragebogens vermerkt werden konnte, welcher Arzt geantwortet hat. Das Deckblatt wurde direkt nach Ankunft des Briefes im Institut der Allgemeinmedizin vom Fragebogen getrennt und separat abgeheftet, sodass eine Zuordnung der Teilnehmer mit dem jeweiligen Fragebogen nicht möglich war (siehe 4.7).

\section{Fragebogen}

\section{Frankierter, adressierter Rückumschlag}

Um die Arbeitsbelastung der Ärzte möglichst gering zu halten, wurde ein frankierter Rückumschlag mit der Adresse des Instituts für Allgemeinmedizin Göttingen beigelegt.

\subsubsection{Zweites Anschreiben}

Alle Ärzte, die bis zum 13.01.2015 nicht geantwortet haben, wurden am 14.01.2015 ein zweites Mal angeschrieben. Es wurde folgendes Material versendet:

- Informationsschreiben 2 (siehe Anlage 9.4)

- Fragebogen (siehe Anlage 9.3)

- Kurzfragebogen (siehe Anlage 9.5)

- Frankierter, adressierter Rückumschlag.

Der Fragebogen und der Rückumschlag waren identisch mit denen des ersten Anschreibens. Das Informationsschreiben wurde verändert, außerdem wurde ein Kurzfragebogen mit versendet. Auf ein Deckblatt wurde verzichtet, da ein drittes Anschreiben nicht durchgeführt werden sollte.

\subsection{Datenanalyse}

Bei dieser Studie handelt es sich um eine Querschnittsstudie an praktizierenden Hausärzten, hausärztlich tätigen Internisten sowie praktischen Ärzten aus Niedersachsen. Bei einer erwartenden Rücklaufquote von 40\% (siehe 4.4.1) wurden 1000 Hausärzte angeschrieben. Die Antwortmöglichkeiten wurden kodiert (siehe Anlage 9.9) und in eine Exceltabelle übertragen. Um mögliche Fehler bei der Übertragung der Angaben in die Exceltabelle zu minimieren, wurden häufige Arbeitszeiten von kurzer Dauer gewählt. 
Die statistische Auswertung erfolgte mit dem Statistikprogramm Statistical Analysis System (SAS) in der Version 9.3 für Windows. Für die relevanten Parameter wurden die relativen und absoluten Häufigkeiten berechnet und - wenn sinnvoll - 95\%-Clopper-PearsonKonfidenzintervalle bestimmt. Logistische Regressionsmodelle wurden verwendet, um den Einfluss verschiedener Variablen auf den Wunsch nach einer Ethikberatung und die Sinnhaftigkeit einer Ethikberatung zu untersuchen. Die Ergebnisse sind in Form von Odds Ratios samt 95\% Konfidenzintervall dargestellt (siehe Tabelle 6 und Tabelle 7). Mit der multiplen logistischen Regression wurden über univariate und multivariate Modelle (Stokes et al. 2012) der Einfluss verschiedener Variablen mit dem Wunsch nach einer Ethikberatung und der Sinnhaftigkeit einer Ethikberatung untersucht und die Odds Ratios mit ihren 95\%Konfidenzintervallen berechnet (siehe 5.7 und 5.8).

\subsection{Umkodierung und Filterführung}

Fragen, die nicht beantwortet wurden, wurden zunächst system-intern als „99“ codiert (siehe Anlage 9.9) und anschließend als „missing“ gesetzt.

Fragen, die trotz Filterführung (siehe 4.2) fälschlicherweise beantwortet wurden, flossen nicht in die Auswertung mit ein.

Halten Sie die Etablierung einer ambulanten Ethikberatung für sinnvoll?
$\square_{1} \mathrm{ja}$
$\square_{2}$ bin mir nicht sicher
$\square_{0}$ nein

[falls nein, weiter mit Frage 13]

Abbildung 7: Umcodierung von Fragen

Die Antworten der Ärzte, die in obiger Frage „nein“ angaben (siehe Anlage 9.3, Frage 6) die Fragen 9 - 12 trotz dessen beantworteten, wurden als „,77“ codiert. Gleiches gilt für Frage 3 und Frage 5 (siehe Anlage 9.3).

Einige Ärzte kreuzten in einer Frage mehrere Antwortmöglichkeiten an, obwohl keine Mehrfachantwort vorgesehen war. Wenn möglich, wurde die mittlere Antwort gewertet (siehe Abbildung 8). Wurden zwei Kreuze gesetzt, wurde die Angabe gewertet, die insgesamt häufiger genannt wurde. 


\begin{tabular}{|c|c|c|c|c|c|}
\hline \multicolumn{6}{|c|}{ Wie oft sind Sie in Ihrer hausärztlichen Tätigkeit mit folgenden } \\
\hline & $\begin{array}{l}\text { ca. 1-mal } \\
\text { im } \\
\text { Monat }\end{array}$ & $\begin{array}{l}\text { ca.1-mal } \\
\text { alle } \\
3 \text { Monate }\end{array}$ & $\begin{array}{l}\text { ca. } 1-\text { mal } \\
\text { alle } \\
6 \text { Monate }\end{array}$ & $\begin{array}{l}\text { seltener } \\
\text { als alle } \\
6 \text { Monate }\end{array}$ & $\begin{array}{l}\text { bisher } \\
\text { nicht } \\
\text { erlebt }\end{array}$ \\
\hline $\begin{array}{l}\text { Fortsetzung einer potentiell } \\
\text { kurativen Therapie, die } \\
\text { schlecht vertragen wird. }\end{array}$ & $\square_{5}$ & $\square_{4}$ & $\boldsymbol{\otimes}_{3}$ & $\boldsymbol{\otimes}_{2}$ & $\boldsymbol{\otimes}_{1}$ \\
\hline
\end{tabular}

Abbildung 8: Mehrfach angekreuzte Möglichkeiten

In Frage 12 wurde nach der Vergütung einer Ethikberatung gefragt; ein Teilnehmer antwortete mit „ja“ und „bin mir nicht sicher“. Diese Antwortmöglichkeit wurde zu „bin mir nicht sicher“ gezählt (siehe Anlage 9.9).

Gefragt nach Ihrem Alter (Frage 14, Anlage 9.3) antworteten drei Ärzte „>50“, „>55“ und „ü60“. Die Antworten „>50“ und „>55“ wurden als fehlend gewertet, die Antwort „ü60“ wurde als 62 gewertet.

In Frage 17 (siehe Anlage 9.3) ergänzten zwei Ärzte handschriftlich die Antwortmöglichkeit „MVZ“. Diese Angabe wurde zu der Antwortmöglichkeit „Praxisgemeinschaft“ gezählt.

Antwortangaben, die keiner vorgegebenen Antwort sinnvoll zugeordnet werden konnten, wurden als missing gesetzt.

\subsection{Datenschutz und Ethik}

Einerseits sollte die Anonymität bei der Auswertung der Fragebögen garantiert sein, andererseits sollte ein doppeltes Anschreiben der Ärzte vermieden werden, die bereits an der ersten Umfrage teilgenommen hatten. Um beides zu gewährleisten, wurde folgendermaßen vorgegangen: An den Fragebogen des ersten Anschreibens wurde ein Deckblatt geheftet (siehe Anlage 9.2), auf dem die Adresse des teilnehmenden Arztes stand. Bei Rücksendung des Bogens an das Institut für Allgemeinmedizin Göttingen wurde das Deckblatt umgehend von dem Fragebogen abgetrennt. Fragebogen und Deckblatt wurden in unterschiedlichen Ordnern abgeheftet, so dass eine Zuordnung zwischen der Adresse des Arztes und seinen Antworten bereits zu diesem Zeitpunkt nicht mehr möglich war. Mit Abschluss des ersten Anschreibens wurden die Deckblätter vernichtet. Der Fragebogen erfragte Geschlecht und Alter des Teilnehmers, seit wann er in der ambulanten Patientenversorgung tätig ist und die Praxisart (siehe 4.2). Es wurden keine Adressen, Namen, Geburtsorte oder weitere Daten, die eine Zuordnung zwischen Antwortbogen und Teilnehmer zulassen würden, erhoben.

Die Eingabe in eine Datenbank erfolgte ebenfalls anonym, sämtliche Daten wurden codiert eingegeben (siehe Anlage 9.9). 
Die beschriebenen Tätigkeiten wurden einzig vom Doktoranden durchgeführt, neben ihm und den Mitarbeiter des Instituts für Allgemeinmedizin Göttingen hatte keine weitere Person Zugriff auf die Daten.

Die Ethikkommission der Universität Göttingen genehmigte das Vorgehen dieser Dissertation (Antragsnummer 11/6/13). 


\section{Ergebnisse}

\subsection{Pilotphase}

In der Pilotphase wurde der Fragebogen an neun Ärzte aus Varel gesendet. Den Hauptfragebogen (siehe Anlage 9.6) beantworteten sechs Ärzte, zwei weitere sendeten den Kurzfragebogen (siehe Anlage 9.5) zurück. Ein Arzt antwortete auch auf das zweite Anschreiben nicht. Telefonisch begründete er die Nichtteilnahme mit fehlender Zeit.

Bei der Auswertung der Pilotphase waren zwei Dinge von besonderer Relevanz:

1. Die Frage nach weiteren konflikthaften Situationen: Neben dem „Absetzen von Arzneien, insbesondere präventiv eingenommen“ wurde der Konflikt „differente Ansichten zu diagnostischen oder therapeutischen Vorgehen zwischen mitbetreuender stationärerer Einrichtung und mir" wahrgenommen (siehe Abbildung 9):

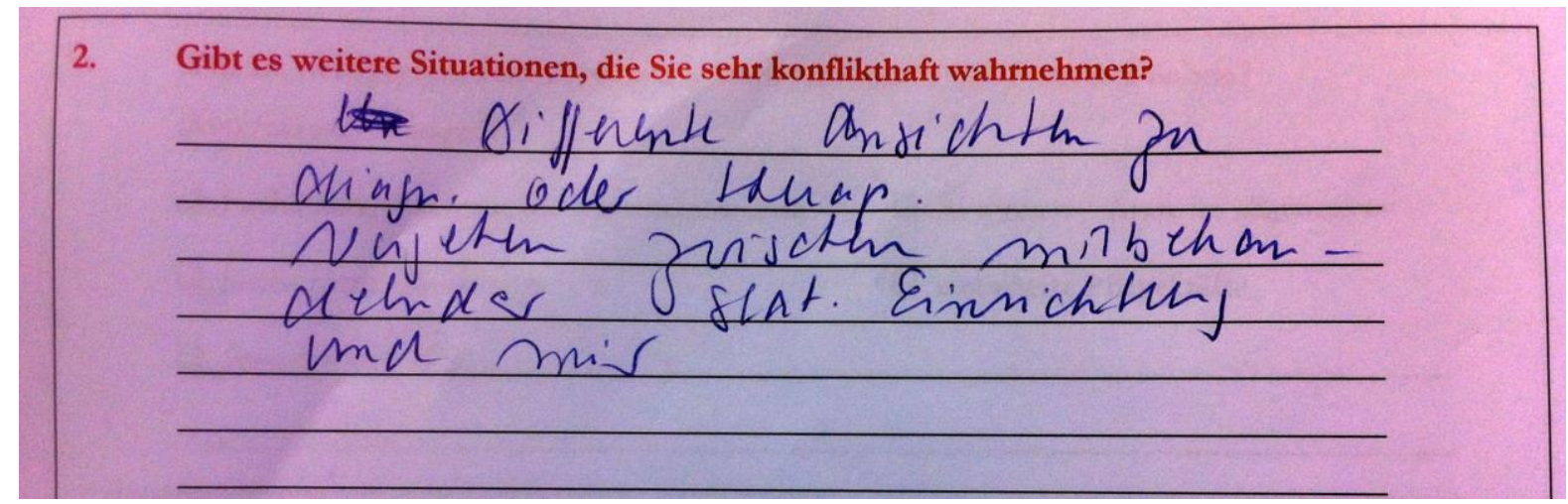

Abbildung 9: Weitere konflikthafte Situationen

Diese Antworten wurden anschließend in den Hauptfragebogen integriert, so dass sie in der Hauptumfrage bewertet werden konnten. 
2. Die Häufigkeitsangaben der Konflikte: Es fiel unter anderem auf, dass die Maximalvariante „häufiger als 1-mal im Monat“ kaum angekreuzt wurde, die Variante „seltener als 1-mal im halben Jahr" dagegen häufig. Die Zeitangaben wurden modifiziert, wie in Abbildung 10 dargestellt:

Wie oft sind Sie in Ihrer hausärztlichen Tätigkeit mit folgenden

Entscheidungskonflikten konfrontiert?

\begin{tabular}{|c|c|c|c|c|}
\hline $\begin{array}{c}\text { häufiger als } \\
\text { 1-mal im } \\
\text { Monat }\end{array}$ & $\begin{array}{l}\text { ca. 1-mal } \\
\text { im } \\
\text { Monat }\end{array}$ & $\begin{array}{l}\text { ca. 1-mal } \\
\text { im } \\
\text { Vierteljahr }\end{array}$ & $\begin{array}{c}\text { ca. 1-mal } \\
\text { im halben } \\
\text { Jahr }\end{array}$ & $\begin{array}{l}\text { seltener als } \\
1 \text {-mal im } \\
\text { halben Jahr }\end{array}$ \\
\hline
\end{tabular}

Wie oft sind Sie in Ihrer hausärztlichen Tätigkeit mit folgenden Entscheidungskonflikten konfrontiert?

$\begin{array}{ccccc}\text { ca. 1-mal } & \text { ca. 1-mal } & \text { ca. 1-mal } & \text { seltener als } & \text { bisher nicht } \\ \text { im } & \text { alle } & \text { alle } & \text { alle } & \text { erlebt } \\ \text { Monat } & 3 \text { Monate } & 6 \text { Monate } & 6 \text { Monate } & \end{array}$

Abbildung 10: Ursprünglicher und veränderter Fragebogen (Hauptumfrage, Frage 1)

\subsection{Ausschöpfungsquote der Umfrage}

Für die Hauptumfrage wurden 1000 Ärzte aus Niedersachsen angeschrieben. Ärzte, die auf das erste Anschreiben nicht reagierten, wurden ein zweites Mal angeschrieben. Dem zweiten Anschreiben wurde ein Kurzfragebogen beigelegt (siehe 4.4.2 und 4.4.3). Insgesamt sendeten 456 (45,6\%) Ärzte den beantworteten Hauptfragebogen zurück, 119 (11,9\%) den Kurzfragebogen (siehe Abbildung 11 und 5.3). Damit reagierten 575 (57,5\%) der befragten Ärzte auf die Umfrage. 


\section{0 angeschriebene Hausärzte aus Niedersachen} (318 Ärztinnen, 682 Ärzte)

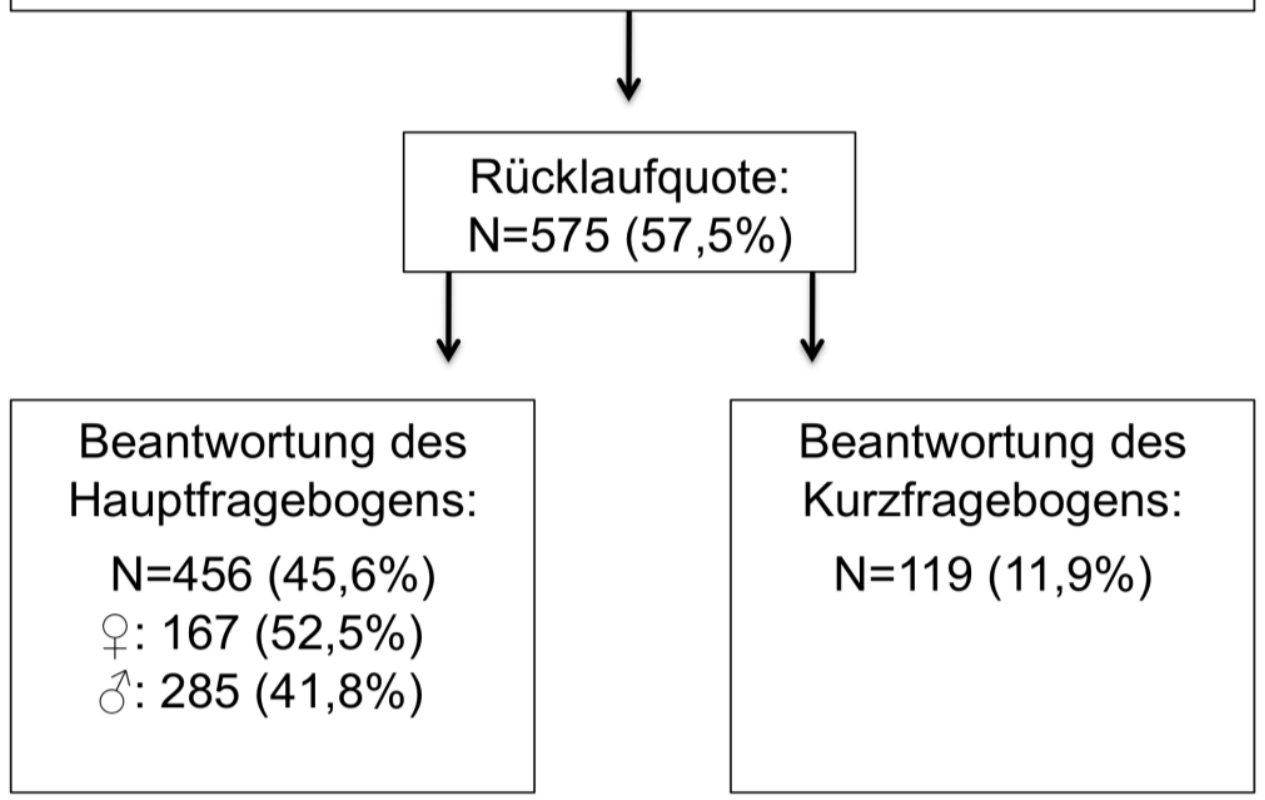

Abbildung 11: Ausschöpfungsquote der Umfrage

\subsection{Beantwortung des Kurzfragebogens}

119 Ärzte beantworteten den Kurzfragebogen und benannten folgende Gründe für die Ablehnung der Teilnahme an der Beantwortung des Hauptfragebogens: Knapp über die Hälfte $(61 / 119 \approx 51,2 \%)$ gab an, dass sie keine Zeit hätten, den Hauptfragebogen zu beantworten. Etwa 40\% (46/119) kreuzten an, zu oft mit Fragebögen konfrontiert zu seien und keine Ressourcen für die Beantwortung aufwenden zu möchten. Rund 20\% (23/119) aller Ärzte würden eine Ethikberatung nicht nutzen. 24 Ärzte machten handschriftliche Angaben, warum sie an der Umfrage nicht teilnehmen können: der Großteil gab an, dass sie nicht mehr als „klassischer" Hausarzt arbeiten würden, sondern andere Schwerpunkte wie Psychotherapie oder Diabetologie hätten.

\subsection{Beschreibung der Teilnehmer-Stichprobe des Hauptfragebogens}

Von den 1000 angeschriebenen Ärzten waren 318 Frauen und 682 Männer. Es antworteten 167 Frauen (52,5\%) und 285 Männer (41,8\%). Die Rücklaufquote unter den Ärztinnen war damit vergleichsweise höher als die der befragten Ärzte. Vier Teilnehmer gaben kein Geschlecht an. Knapp 40\% (168/456) der Ärzte praktizieren in Einzelpraxen, 10,5\% in Praxis- 
gemeinschaften; 52,1\% arbeiten in Gemeinschaftspraxen. Das Durchschnittsalter der Teilnehmer beträgt 54,0 Jahre (SD: 8,3; min: 31; max: 73); in der ambulanten Versorgung arbeiten sie seit durchschnittlich 18,7 (min: 1; max: 54) Jahren (siehe Tabelle 2).

Tabelle 2: Tätigkeitsdauer in der ambulanten Versorgung*

\begin{tabular}{lll}
\hline In der ambulanten Versorgung seit (Jahren) & $\mathrm{n}$ & $\%$ \\
\hline $0-4$ & 30 & 6,7 \\
\hline $5-9$ & 63 & 14,0 \\
\hline $10-14$ & 75 & 16,7 \\
\hline $15-19$ & 80 & 17,8 \\
\hline $20-24$ & 66 & 14,7 \\
\hline $25-29$ & 63 & 14,0 \\
\hline $30-34$ & 50 & 11,1 \\
\hline ab 35 & 22 & 4,9 \\
\hline
\end{tabular}

${ }^{\star}$ Basis: $n=449$

\subsection{Ethische Konflikte in Hausarztpraxen}

Im Zentrum der Befragung standen Art und Häufigkeit ethischer Konflikte in der hausärztlichen Versorgung. Im Folgenden sind die abgefragten ethischen Konflikte tabellarisch dargestellt, zur besseren Übersicht als zusammengefasste Häufigkeiten (Tabelle 3) und aufgeschlüsselt nach Geschlecht und in fünf Häufigkeitskategorien (Tabelle 4).

Häufig erlebte Konflikte wurden definiert als Konflikte, die von den Befragten einmal alle 3 Monate oder öfter erlebt werden (siehe Tabelle 3). Folgende Konflikte wurden dieser Definition nach häufig erlebt:

1. Die Fortsetzung einer potentiellen kurativen Therapie, die schlecht vertragen wird $(67,8 \%)$.

2. Das Absetzen von Arzneien (80,0\%;).

3. Diagnostische Maßnahmen zur Früherkennung bei bereits schwerkranken Patienten $(62,3 \%)$.

Aufgeschlüsselt nach Geschlecht zeigte sich ein ähnliches Ergebnis, wobei der Konflikt des Absetzens von Arzneien hervorsticht; 44,3\% der Ärztinnen und 55,3\% der Ärzte erlebten inn mindestens einmal pro Monat (Tabelle 4). 
Tabelle 3: Ethische Konflikte

Wie oft sind Sie in Ihrer hausärztlichen Tätigkeit mit folgenden Entscheidungskonflikten konfrontiert?

\begin{tabular}{|c|c|c|}
\hline Konflikt & Teilnehmer (n) & Häufigkeit in \%* $\left(95 \% \mathrm{KI}^{* *}\right)$ \\
\hline $\begin{array}{l}\text { Fortsetzung einer potentiell } \\
\text { kurativen Therapie, die schlecht } \\
\text { vertragen wird. }\end{array}$ & 453 & $67,8(63,3-72,0)$ \\
\hline $\begin{array}{l}\text { Fortsetzung einer potentiell } \\
\text { lebensverlängernden Therapie } \\
\text { bei infauster Prognose, die } \\
\text { schlecht vertragen wird. }\end{array}$ & 455 & $41,5(37,0-46,2)$ \\
\hline $\begin{array}{l}\text { Fortsetzung einer Therapie, } \\
\text { wenn der Patientenwille wegen } \\
\text { einer progredienten Erkrankung } \\
\text { nicht mehr feststellbar ist. }\end{array}$ & 455 & $25,1(21,1-29,3)$ \\
\hline $\begin{array}{l}\text { Informationsweitergabe an An- } \\
\text { gehörige, wenn nicht bekannt } \\
\text { ist, ob der Patient dies gestattet } \\
\text { hat. }\end{array}$ & 456 & $40,4(35,8-45,0)$ \\
\hline $\begin{array}{l}\text { Nachträgliches Umsetzen einer } \\
\text { Patientenverfügung, die in einer } \\
\text { Notfallsituation nicht berücksich- } \\
\text { tigt wurde. }\end{array}$ & 456 & $7,5(5,2-10,3)$ \\
\hline $\begin{array}{l}\text { Einweisung von pflegebedürfti- } \\
\text { gen o. wesensveränderten Pati- } \\
\text { enten in Pflegeeinrichtungen } \\
\text { gegen deren Willen. }\end{array}$ & 456 & $13,6(10,6-17,1)$ \\
\hline $\begin{array}{l}\text { Selbstgefährdendes Patienten- } \\
\text { verhalten. }\end{array}$ & 453 & $36,9(32,4-41,5)$ \\
\hline $\begin{array}{l}\text { Vorgehen bei strafrechtlich rele- } \\
\text { vanten Informationen über Dritte } \\
\text { (beispielsweise häusliche Ge- } \\
\text { walt durch den Partner). }\end{array}$ & 454 & $6,4(4,3-9,1)$ \\
\hline $\begin{array}{l}\text { Konträre Behandlungsansichten } \\
\text { zwischen mir und einer stationä- } \\
\text { ren Einrichtung. }\end{array}$ & 456 & $47,6(42,9-52,3)$ \\
\hline $\begin{array}{l}\text { Absetzen von Arzneien bei mitt- } \\
\text { lerweile fraglichem Nutzen für } \\
\text { den Patienten. }\end{array}$ & 455 & $80,0(76,0-83,6)$ \\
\hline $\begin{array}{l}\text { Sinnhaftigkeit diagnostischer } \\
\text { Maßnahmen zur Früherkennung } \\
\text { bei bereits schwerkranken Pati- } \\
\text { enten. }\end{array}$ & 456 & $62,3(57,7-66,8)$ \\
\hline $\begin{array}{l}\text { Patienten und Angehörigen sind } \\
\text { uneins bzgl. diagnostischer } \\
\text { bzw. therapeutischer Maßnah- } \\
\text { men. }\end{array}$ & 456 & $52,9(48,2-57,5)$ \\
\hline
\end{tabular}

${ }^{*}$ Anteil der Teilnehmer, die den Konflikt ca. einmal alle 3 Monate oder häufiger erlebten

${ }^{* *}$ Konfidenzintervall 
Tabelle 4: Häufigkeit ethischer Konflikte; nach Geschlecht

Wie oft sind Sie in Ihrer hausärztlichen Tätigkeit mit folgenden Entscheidungskonflikten konfrontiert?

\begin{tabular}{|c|c|c|c|c|c|c|}
\hline \multirow[t]{2}{*}{ Konflikt } & \multirow{2}{*}{$\begin{array}{l}\text { Geschlecht } \\
\text { und Teil- } \\
\text { nehmer }(\mathrm{n})\end{array}$} & \multicolumn{5}{|c|}{ Häufigkeit in \% } \\
\hline & & $\begin{array}{l}\text { ca. } 1 \mathrm{x} \\
\text { im } \\
\text { Monat }\end{array}$ & $\begin{array}{l}\text { ca. } 1 \mathrm{x} \\
\text { alle } 3 \\
\text { Monate }\end{array}$ & $\begin{array}{l}\text { ca. } 1 \mathrm{x} \\
\text { alle } 6 \\
\text { Monate }\end{array}$ & $\begin{array}{l}\text { seltener } \\
\text { als alle } 6 \\
\text { Monate }\end{array}$ & $\begin{array}{l}\text { bisher } \\
\text { nicht } \\
\text { erlebt }\end{array}$ \\
\hline $\begin{array}{l}\text { Fortsetzung einer potentiell kura- } \\
\text { tiven Therapie, die schlecht ver- } \\
\text { tragen wird. }\end{array}$ & $\begin{array}{l}\text { or: } 167 \\
\text { ôt: } 282\end{array}$ & $\begin{array}{l}34,7 \\
52,1\end{array}$ & $\begin{array}{l}28,7 \\
17,7\end{array}$ & $\begin{array}{l}16,2 \\
15,6\end{array}$ & $\begin{array}{l}16,2 \\
12,8\end{array}$ & $\begin{array}{l}4,2 \\
1,8\end{array}$ \\
\hline $\begin{array}{l}\text { Fortsetzung einer potentiell le- } \\
\text { bensverlängernden Therapie bei } \\
\text { infauster Prognose, die schlecht } \\
\text { vertragen wird. }\end{array}$ & $\begin{array}{l}o: 167 \\
\text { o } \\
0\end{array}$ & $\begin{array}{l}15,6 \\
19,4\end{array}$ & $\begin{array}{l}19,8 \\
25,4\end{array}$ & $\begin{array}{l}25,8 \\
23,6\end{array}$ & $\begin{array}{l}28,7 \\
26,8\end{array}$ & $\begin{array}{c}10,2 \\
4,9\end{array}$ \\
\hline $\begin{array}{l}\text { Fortsetzung einer Therapie, wenn } \\
\text { der Patientenwille wegen einer } \\
\text { progredienten Erkrankung nicht } \\
\text { mehr feststellbar ist. }\end{array}$ & $\begin{array}{l}\circ: 166 \\
\text { to }\end{array}$ & $\begin{array}{c}9,0 \\
11,2\end{array}$ & $\begin{array}{l}15,7 \\
14,4\end{array}$ & $\begin{array}{l}22,9 \\
18,6\end{array}$ & $\begin{array}{l}36,1 \\
46,0\end{array}$ & $\begin{array}{c}16,3 \\
9,8\end{array}$ \\
\hline $\begin{array}{l}\text { Informationsweitergabe an Ange- } \\
\text { hörige, wenn nicht bekannt ist, ob } \\
\text { der Patient dies gestattet hat. }\end{array}$ & $\begin{array}{l}\rho: 167 \\
\text { N: }: 285\end{array}$ & $\begin{array}{l}22,2 \\
23,5\end{array}$ & $\begin{array}{l}16,8 \\
18,3\end{array}$ & $\begin{array}{l}25,2 \\
22,8\end{array}$ & $\begin{array}{l}21,0 \\
26,3\end{array}$ & $\begin{array}{c}15,0 \\
9,1\end{array}$ \\
\hline $\begin{array}{l}\text { Nachträgliches Umsetzen einer } \\
\text { Patientenverfügung, die in einer } \\
\text { Notfallsituation nicht berücksich- } \\
\text { tigt wurde. }\end{array}$ & 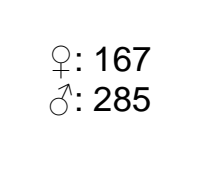 & $\begin{array}{l}1,8 \\
2,5\end{array}$ & $\begin{array}{l}3,6 \\
6,3\end{array}$ & $\begin{array}{l}7,8 \\
8,8\end{array}$ & $\begin{array}{l}35,3 \\
35,1\end{array}$ & $\begin{array}{l}51,5 \\
47,4\end{array}$ \\
\hline $\begin{array}{l}\text { Einweisung von pflegebedürftigen } \\
\text { o. wesensveränderten Patienten } \\
\text { in Pflegeeinrichtungen gegen } \\
\text { deren Willen. }\end{array}$ & $\begin{array}{l}\text { P: } 167 \\
\text { on }: 285\end{array}$ & $\begin{array}{l}2,4 \\
3,2\end{array}$ & $\begin{array}{r}9,0 \\
11,9\end{array}$ & $\begin{array}{l}16,8 \\
25,3\end{array}$ & $\begin{array}{l}52,7 \\
46,3\end{array}$ & $\begin{array}{l}19,2 \\
13,3\end{array}$ \\
\hline $\begin{array}{l}\text { Selbstgefährdendes Patienten- } \\
\text { verhalten. }\end{array}$ & $\begin{array}{l}\text { op: } 165 \\
\text { N: } 284\end{array}$ & $\begin{array}{l}18,2 \\
14,8\end{array}$ & $\begin{array}{l}17,0 \\
22,9\end{array}$ & $\begin{array}{l}20,6 \\
23,2\end{array}$ & $\begin{array}{l}37,6 \\
38,4\end{array}$ & $\begin{array}{l}6,7 \\
0,7\end{array}$ \\
\hline $\begin{array}{l}\text { Vorgehen bei strafrechtlich rele- } \\
\text { vanten Informationen über Dritte } \\
\text { (beispielsweise häusliche Gewalt } \\
\text { durch den Partner). }\end{array}$ & 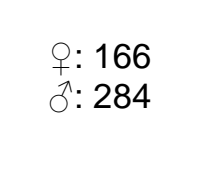 & $\begin{array}{l}0,0 \\
2,1\end{array}$ & $\begin{array}{l}5,4 \\
4,9\end{array}$ & $\begin{array}{l}10,2 \\
12,3\end{array}$ & $\begin{array}{l}50,6 \\
59,5\end{array}$ & $\begin{array}{l}33,7 \\
21,1\end{array}$ \\
\hline $\begin{array}{l}\text { Konträre Behandlungsansichten } \\
\text { zwischen mir und einer stationä- } \\
\text { ren Einrichtung. }\end{array}$ & $\begin{array}{l}o: 167 \\
+: 1285\end{array}$ & $\begin{array}{l}15,0 \\
23,9\end{array}$ & $\begin{array}{l}25,2 \\
27,2\end{array}$ & $\begin{array}{l}21,6 \\
17,5\end{array}$ & $\begin{array}{l}29,3 \\
23,5\end{array}$ & $\begin{array}{l}9,0 \\
7,4\end{array}$ \\
\hline $\begin{array}{l}\text { Absetzen von Arzneien bei mitt- } \\
\text { lerweile fraglichem Nutzen für } \\
\text { den Patienten. }\end{array}$ & $\begin{array}{l}\text { o: } 167 \\
\text { ôt: } 284\end{array}$ & $\begin{array}{l}44,3 \\
55,3\end{array}$ & $\begin{array}{l}30,0 \\
27,8\end{array}$ & $\begin{array}{l}17,4 \\
13,4\end{array}$ & $\begin{array}{l}7,2 \\
2,8\end{array}$ & $\begin{array}{l}1,2 \\
0,7\end{array}$ \\
\hline $\begin{array}{l}\text { Sinnhaftigkeit diagnostischer } \\
\text { Maßnahmen zur Früherkennung } \\
\text { bei bereits schwerkranken Pati- } \\
\text { enten. }\end{array}$ & $\begin{array}{l}o: 167 \\
\text { P: } 1: 285\end{array}$ & $\begin{array}{l}26,4 \\
37,9\end{array}$ & $\begin{array}{l}29,9 \\
27,4\end{array}$ & $\begin{array}{l}22,2 \\
19,0\end{array}$ & $\begin{array}{l}15,0 \\
12,3\end{array}$ & $\begin{array}{l}6,6 \\
3,5\end{array}$ \\
\hline $\begin{array}{l}\text { Patienten und Angehörigen sind } \\
\text { uneins bzgl. diagnostischer bzw. } \\
\text { therapeutischer Maßnahmen. }\end{array}$ & $\begin{array}{l}\text { or: } 167 \\
\text { on: } 285\end{array}$ & $\begin{array}{l}13,2 \\
22,8\end{array}$ & $\begin{array}{l}31,7 \\
34,4\end{array}$ & $\begin{array}{l}24,0 \\
22,1\end{array}$ & $\begin{array}{l}24,6 \\
17,5\end{array}$ & $\begin{array}{l}6,6 \\
3,2\end{array}$ \\
\hline
\end{tabular}




\subsection{Teilnahme an Qualitätszirkeln und Balintgruppen und deren Unterstützung in konflikthaften Situationen}

Zwei Drittel (298/456) der Befragten nahmen an Qualitätszirkeln teil, 54,7\% haben dort Unterstützung in konflikthaften Situationen erhalten; 29\% hielten eine Unterstützung bisher für nicht nötig. An Supervisions- oder Balintgruppen nahmen 21\% (96/450) der Ärzte teil. Die Mehrheit $(85,3 \%)$ von innen hat dort Unterstützung in konflikthaften Situationen erhalten, $10,5 \%$ hielten eine Unterstützung bisher für nicht nötig.

\subsection{Wunsch nach Ethikberatung}

Etwa 30\% (138/451) aller Befragten wünschten sich eine Ethikberatung, 37\% (168/451) wünschten sich keine Ethikberatung; ein Drittel (145/451) der Ärzte waren sich nicht sicher (siehe Tabelle 5). Während sich knapp 38\% (62/164) der Ärztinnen eine Ethikberatung wünschten, sind es bei den Ärzten 26\% (74/283). Die Teilnehmer, die sich eine Ethikberatung wünschten, waren durchschnittlich 53,9 Jahre alt.

Tabelle 5: Wunsch nach einer Ethikberatung

\begin{tabular}{lccc}
\hline $\begin{array}{l}\text { Wunsch } \\
\text { Ethikberatung } \\
(\mathrm{n}=451)\end{array}$ & Teilnehmer $(\mathrm{n})$ & Prozent (\%) & $\begin{array}{c}\text { Mittleres Alter in } \\
\text { Jahren }\end{array}$ \\
\hline $\mathrm{Ja}$ & 138 & 30,6 & 53,9 \\
\hline Bin mir nicht sicher & 145 & 32,2 & 53,1 \\
\hline Nein & 168 & 37,3 & 54,9 \\
\hline
\end{tabular}

Im Rahmen der univariaten logistischen Regression waren die Parameter Geschlecht (OR 1,72, 95\% KI 1,14-2,59), die Vergütung mit der Antwortmöglichkeit „bin mir nicht sicher” (OR $0,4195 \% \mathrm{KI}$ 0,23-0,71) und die Teilnahme an einer Supervisionsgruppe (OR 1,64, 95\% KI 1,02-2,63) signifikant bezüglich des Wunsches nach einer Ethikberatung (siehe Tabelle 6). Zusätzlich wurde ein multivariates Regressionsmodell angepasst. Eingang in die Berechnung fanden die Parameter Geschlecht, Praxisart, Qualitätszirkel, Supervisionsgruppe, Vergütung, Alter und Patientenversorgung. In der Backward-Selection spielten die Parameter Geschlecht mit der Referenzgruppe „Ärzte“, Vergütung (,ja“ und „bin mir nicht sicher“) und die Teilnahme an einer Supervisionsgruppe eine Rolle in Bezug auf den Wunsch nach einer Ethikberatung. Signifikant war einzig die Vergütung mit einer OR von 0,42 (KI 0,24-0,74) bezüglich der Antwortmöglichkeit „bin mir nicht sicher" (siehe Tabelle 6). 
Tabelle 6: Einflussfaktoren im Hinblick auf den Wunsch nach einer Ethikberatung

\begin{tabular}{|c|c|c|c|c|c|}
\hline \multirow[b]{2}{*}{ Faktoren } & \multirow[b]{2}{*}{ Wunsch** (in \%) } & \multicolumn{2}{|c|}{ Univariates Modell } & \multicolumn{2}{|c|}{ Multivariates Modell* } \\
\hline & & $\mathrm{OR}^{\star * \star}\left(95 \% \mathrm{KI}^{* \star * *}\right)$ & $p$ & $\mathrm{OR}^{* * *}\left(95 \% \mathrm{KI}^{* * * *}\right)$ & $p$ \\
\hline \multicolumn{6}{|l|}{ Geschlecht } \\
\hline Ärzte & 26,2 & & & & \\
\hline Ärztinnen & 37,8 & $1.72(1,14-2,59)$ & 0,01 & $1,46(0,94-2,27)$ & 0,091 \\
\hline \multicolumn{6}{|l|}{ Praxisart } \\
\hline Einzelpraxis & 28,3 & & & & \\
\hline Mehrere Ärzte & 31,7 & $1,17(0,77-1,79)$ & 0,459 & & \\
\hline \multicolumn{6}{|c|}{ wünscht Vergütung } \\
\hline $\mathrm{Ja}$ & 45,3 & $1,4(0,85-2,3)$ & 0,189 & $1,33(0,8-2,23)$ & 0,27 \\
\hline Nein & 37,2 & & & & \\
\hline Unsicher & 19,4 & $0,41(0,23-0,71)$ & 0,001 & $0,42(0,24-0,74)$ & 0,003 \\
\hline \multicolumn{6}{|c|}{ ist im Qualitätszirkel } \\
\hline $\mathrm{Ja}$ & 31,9 & $1,19(0,78-1,82)$ & 0,423 & & \\
\hline Nein & 28,2 & & & & \\
\hline \multicolumn{6}{|c|}{ ist in Supervisions- } \\
\hline gruppe & & & & & 0,167 \\
\hline $\mathrm{Ja}$ & 38,5 & $1,64(1,02-2,63)$ & 0,041 & $1,44(0,86-2,39)$ & \\
\hline Nein & 27,7 & & & & \\
\hline \multicolumn{2}{|l|}{ Alter } & $1,0(0,97-1,02)$ & 0,862 & & \\
\hline \multirow{2}{*}{\multicolumn{2}{|c|}{$\begin{array}{l}\text { Dauer der } \\
\text { Patientenversorgung }\end{array}$}} & $1,01(0,99-1,03)$ & 0,504 & & \\
\hline & & & & & \\
\hline \multicolumn{6}{|c|}{ *Backward Selection n } \\
\hline \multicolumn{6}{|c|}{${ }^{* \star}$ Anteil (in \%) derjenigen, die eine Ethikberatung wünschen } \\
\hline \multicolumn{6}{|l|}{ *** Odds-Ratio } \\
\hline${ }^{\star * \star *}$ Konfidenzinte & & & & & \\
\hline
\end{tabular}




\subsection{Ethikberatung sinnvoll}

Tabelle 7: Einflussfaktoren im Hinblick auf die Sinnhaftigkeit einer Ethikberatung

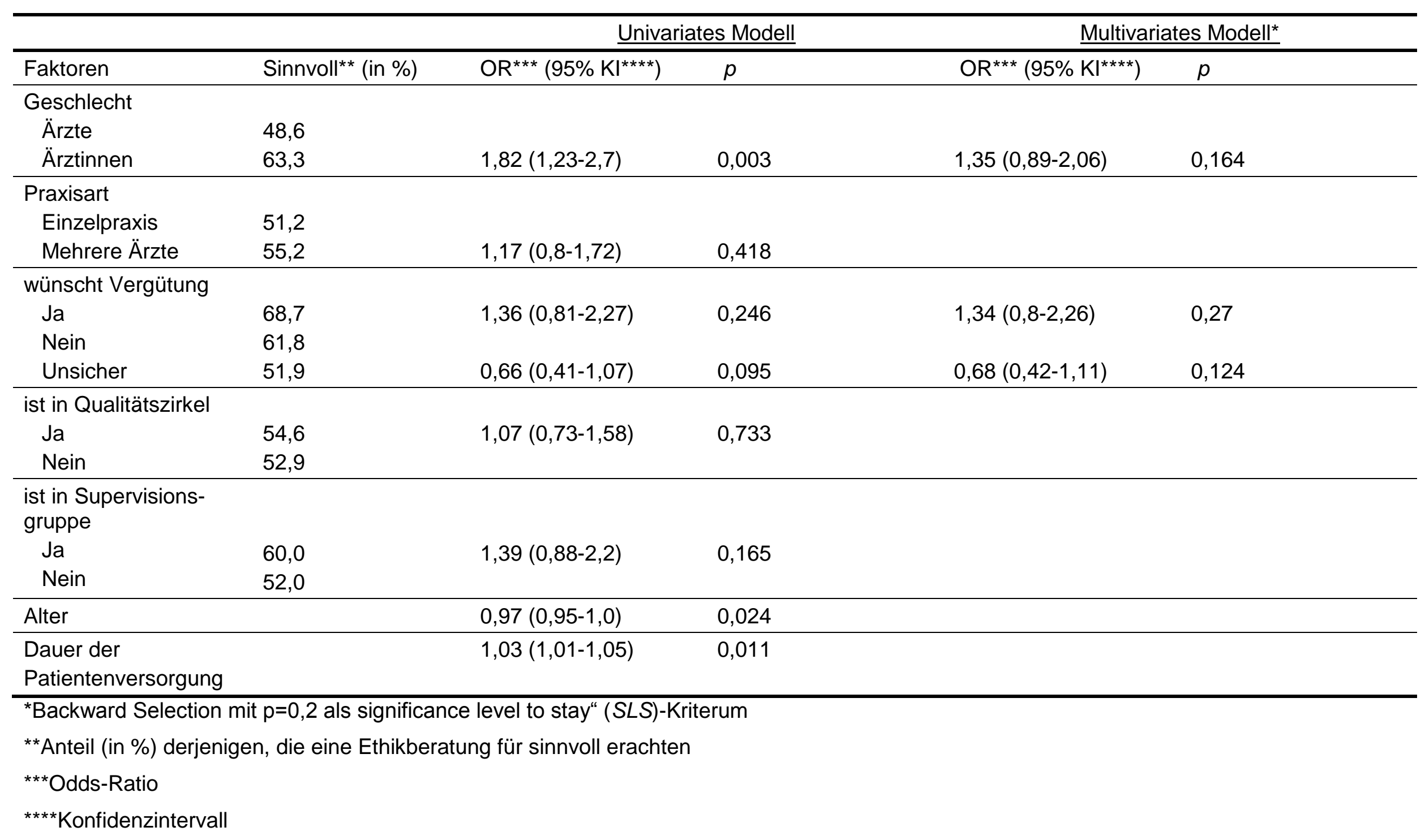


Mehr als die Hälfte (245/454) aller Befragten hielt die Etablierung einer Ethikberatung für sinnvoll (siehe Tabelle 8), häufiger Frauen als Männer (63\% vs. 49\%). Insgesamt $11 \%$ der Teilnehmer fanden die Etablierung einer Ethikberatung nicht sinnvoll, 35\% sind sich unsicher. Die logistische Regression zeigte eine Tendenz, dass jüngere Ärzte eine Ethikberatung eher sinnvoll finden als ihre älteren Kollegen.

Tabelle 8: Ethikberatung wird als sinnvoll erachtet

\begin{tabular}{lccc}
\hline $\begin{array}{l}\text { Ethikberatung } \\
\text { sinnvoll } \\
(\mathrm{n}=454)\end{array}$ & Teilnehmer $(\mathrm{n})$ & Prozent (\%) & $\begin{array}{c}\text { Mittleres Alter in } \\
\text { Jahren }\end{array}$ \\
\hline $\mathrm{Ja}$ & 245 & 54,0 & 53,2 \\
\hline Bin mir nicht sicher & 160 & 35,2 & 54,2 \\
\hline Nein & 49 & 10,8 & 57,4 \\
\hline
\end{tabular}

Im Rahmen der univariaten logistischen Regression zeigte das Geschlecht mit einem OR von 1,82 (95\% KI 1,23-2,7) einen signifikanten Unterschied hinsichtlich der Etablierung einer Ethikberatung (siehe Tabelle 7). Auch die Parameter Alter mit einer OR von 0,97 (95\% KI 0,95-1,0) und die Dauer der Patientenversorgung mit einer OR von 1,03 (95\% KI 1,01$1,05)$ waren signifikant. Eingang in das multivariate Regressionsmodell fanden die Parameter Geschlecht, Praxisart, Qualitätszirkel, Supervisionsgruppe, Vergütung, Alter und Patientenversorgung. Wie schon in der univariaten Auswertung wünschten Frauen häufiger die Etablierung einer Ethikberatung (OR 1,35, 95\% KI 0,89-2,06). Auch die Option, die Ethikberatung würde vergütet, hatte Einfluss. Wenn es eine Vergütung gäbe, würden mehr Ärzte eine Etablierung als sinnvoll erachten (OR 1,34, 95\% KI 0,8-2,26).

\subsection{Angebotswünsche an eine ambulante Ethikberatung}

Die Mehrheit der Ärzte wünschte sich eine telefonische Beratung (327/401 $\approx$ 81,6\%; siehe Abbildung 12) und Fortbildungen zu medizinethischen Fragestellungen (285/401 $\approx 71 \%$ ). Weniger als ein Viertel der Befragten würde die Beratung in der Praxis $(23,7 \%)$ oder am Krankenbett $(22,9 \%)$ in Anspruch nehmen. 


\section{Wünsche an eine ambulante Ethikberatung}

$\mathrm{n}=401$, Häufigkeit in Prozent

Leitlinienerstellung für wiederkehrende medizinische Konfliktsituationen

Fortbildung zu medizinethischen Fragestellungen für Hausärzte

Beratung am Krankenbett

Retrospektive Fallbesprechung

Beratung in der Praxis

Telefonische Beratung

\section{1,9}

71,1

22,9

55,9

23,7

81,6

Abbildung 12: Wünsche an eine ambulante Ethikberatung

\subsection{Berufliche Hintergründe und Qualifikationen eines Ethikberaters}

Über die Hälfte aller Befragten sprach sich für Vertreter aus der Allgemeinmedizin (241/401 $\approx 60,1 \%)$ und Ärzte im Allgemeinen $(224 / 401 \approx 55,9 \%$ ) in Ethikkomitees aus (siehe Tabelle 9); etwa ein Viertel wünschte sich Pflegekräfte in Ethikkomitees (27,9\%).

Eine große Mehrheit der Befragten wünschte sich medizinisches Fachwissen, Erfahrungen in der ambulanten Patientenversorgung und Kenntnisse bezüglich des Medizinrechts von Mitgliedern eines Ethikkomitees (Tabelle 9). 
Tabelle 9: Berufliche Hintergründe und Qualifikationen eines Ethikberaters*

\begin{tabular}{lcc}
\hline $\begin{array}{l}\text { Berufliche Hintergründe } \\
\mathrm{n}=401\end{array}$ & Anzahl & Ja \\
\hline $\begin{array}{l}\text { Fachärztin/ Facharzt für Allgemein- } \\
\text { medizin }\end{array}$ & 241 & 60,1 \\
\hline $\begin{array}{l}\text { Ärztinnen/ Ärzte im Allgemeinen } \\
\text { Seelsorgerin/ Seelsorger }\end{array}$ & 224 & 55,9 \\
\hline $\begin{array}{l}\text { Ambulante Pflegekräfte } \\
\text { Qualifikationen }\end{array}$ & 185 & 46,1 \\
\hline $\mathrm{n}=401$ & 357 & 27,9 \\
\hline $\begin{array}{l}\text { Medizinisches Fachwissen } \\
\text { Erfahrungen in der ambulanten Pa- } \\
\text { tientenversorgung }\end{array}$ & 359 & 89,0 \\
\hline $\begin{array}{l}\text { Kenntnisse bezüglich des Medizin- } \\
\text { rechts }\end{array}$ & 347 & 86,5 \\
\hline
\end{tabular}

*Mehrfachantworten möglich

\subsection{Vergütung der Teilnahme an einer Ethikberatung}

Während 25\% (99/398) der Ärzte eine ambulante Ethikberatung eher in Anspruch nehmen würden, wenn sie vergütet werden würde, verneinten diese Aussage knapp die Hälfte der

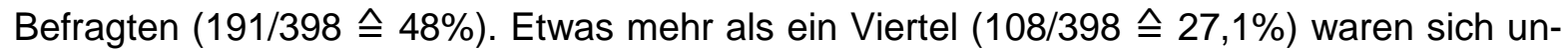
sicher. Das Geschlecht und die Praxisart der Teilnehmer hatten keinen Einfluss auf die Beantwortung der Frage.

\subsection{Auswertung der Freitextfelder des Fragebogens}

Fünf der Fragen wurden durch Freitextfelder erweitert, in denen die Ärzte zusätzliche Angaben machen konnten. Dazu gehört die Frage nach weiteren Konflikten, nach weiteren Angeboten einer ambulanten Ethikberatung, weiteren beruflichen Hintergründen der Ethikberater, weiteren Qualifikationen von Ethikberatern und Zusatzqualifikationen der antwortenden Ärzte. 


\subsubsection{Auswertung weiterer Konflikte}

Das Freitextfeld für weitere Konflikte nutzten $31 \%(n=143)$ der befragten Ärzte. Im Folgenden werden Konflikte beschrieben, die zum Teil mehrfach genannt wurden.

Weitergabe von Patientendaten. Ein Konflikt bestehe in der Weitergabe von Patientendaten an Heime, den Medizinischen Dienst der Krankenkassen und den Arbeitgeber. Auch die Weitergabe von Informationen an Eltern von Jugendlichen zwischen 14 bis 18 Jahren sei ein Konflikt.

Wunsch zu sterben. Der Umgang mit Nahrungsverweigerung als Suizidmittel, die Frage nach einer „Erlösungsspritze“ sowie der Wunsch von Patienten nach aktiver oder passiver Sterbehilfe wurden mehrfach als Konflikt angegeben.

Budgetierung. Die Rationierung von Arzneimitteln und anderen Ressourcen, vor allem bei Schwerkranken, Kosten-Nutzen-Bewertungen von Medikationen, die geringe Vergütung für „sprechende Medizin“ und die Diskrepanz zwischen Werbeversprechen der Krankenkassen gegenüber ihrem Erstattungsverhalten scheinen Ärzte vor eine Herausforderung zu stellen. Es entständen Konflikte zwischen wirtschaftlicher und medizinisch sinnvoller Verordnung, zum Beispiel bei der Verschreibung von Physiotherapie und Massagen.

Anspruchsdenken. Die hohe Erwartungshaltung von Patienten und deren Angehörigen an Ärzte wurde häufig als Konflikt genannt, besonders sogenannte Wunschmedikation, Wunschphysiotherapie, Krankschreibung und uneffektive Präventionsmaßnahmen. Angehörige würden unverhältnismäßige Forderungen stellen und versuchen, die Fürsorgepflicht auf die Hausärzte zu übertragen.

Therapieablehnung und fehlende Compliance. Die Ablehnung von sinnvollen diagnostischen oder therapeutischen Maßnahmen mit Eigengefährdung bei teilweise nicht orientierten Patienten wird gehäuft als Konflikt genannt. Patienten würden sich weigern, stationär aufgenommen zu werden, sie würden Medikamente ablehnen und Alkohol- und Nikotinkonsum fortsetzen.

Therapie bei Demenz. Neben der Wahl des Betreuers für demente Patienten stellt die Fortsetzung von Sondennahrung und die Umsetzung des mutmaßlichen Patientenwillens bei fortgeschrittener Demenz ein Problem dar.

Konträre Therapieempfehlungen zwischen Hausarzt und anderen Ärzten. Vor allem Onkologen - so einige Hausärzte - würden Therapieangebote wie Chemotherapie bei in- 
fauster Prognose nicht abbrechen. Patienten würden trotz Intervention durch den Hausarzt sehr spät in die Häuslichkeit entlassen oder bis zu ihrem Tode behandelt.

Medizin am Lebensende. Häufig wurden Unsicherheiten bei Therapieabbruch von palliativen Patienten sowie der Übergang von kurativer zu palliativer Behandlung genannt. Unklarheiten bei der Umsetzung von Patientenverfügungen sowie der passende Zeitpunkt zur Einstellung von Nahrung, Flüssigkeit oder Dialyse seien problematisch. Auch das Verschweigen einer infausten Prognose auf Wunsch von Angehörigen gegenüber dem Patienten wird als Konflikt empfunden.

\subsubsection{Weitere Angebote einer Ethikberatung}

Das Freitextfeld für weitere Angebote einer Ethikberatung nutzten 23 Ärzte $(23 / 456 \approx$ $0,05 \%$ ). Gehäuft wurde der Wunsch nach einer engeren Zusammenarbeit zwischen stationärem und ambulantem Bereich geäußert. Ein Arzt schlug dafür eine übergreifende Ethikberatung zwischen Krankenhäusern und Hausärzten vor. Außerdem wurde die ständige Erreichbarkeit eines Ethikkomitees, auch nachts und am Wochenende, mehrmals genannt.

\subsubsection{Berufliche Hintergründe und Eigenschaften eines Ethikberaters}

Das Freitextfeld für weitere nutzte knapp ein Viertel $(106 / 456 \approx 23,2 \%)$ der befragten Ärzte. Berufswünsche an Ethikberater, die mehrfach genannt wurden, waren: Juristen, Psychologen, Psychotherapeuten, Theologen, Philosophen und Palliativmedizinern. Einige Ärzte nannten zudem langjährige Berufs,- und Lebenserfahrung als wichtige Eigenschaft.

\subsubsection{Weitere Qualifikationen eines Ethikberaters}

Das Freitextfeld für weitere Qualifikationen nutzten 80 Ärzte (80/456 17,5\%). Die folgenden Kenntnisse wurden als vorteilhaft genannt: juristische, theologische, philosophische, ethische, moralische und empathische Kenntnisse. Von Vorteil seien zudem Kommunikationskompetenz und Lebenserfahrung.

\subsubsection{Zusatzqualifikationen der Teilnehmer}

330 Ärzte $(330 / 456 \approx 72,3 \%)$ gaben berufliche Zusatzqualifikationen an. Zusatzqualifikationen, die mehrfach genannt wurden, waren: Palliativmedizin, psychosomatische Grundversorgung, Psychotherapie und Notfallmedizin. Weniger häufig wurden Qualifikationen wie Flugmedizin, Suchtmedizin, Betriebsmedizin oder Chirotherapie erwähnt. 


\section{Diskussion}

Einige ethische Konflikte, zum Beispiel das „Absetzen von Arzneien bei mittlerweile fraglichem Nutzen für den Patienten“, wurden von der Mehrheit der Hausärzte mindestens einmal im Monat erlebt, andere Konflikte deutlich seltener. Die Mehrheit der Befragten nahm an Qualitätszirkeln teil; etwas mehr als die Hälfte empfand dies als hilfreich für die Lösung ethischer Konflikte. Etwa ein Fünftel der Teilnehmer nahm an Supervisions- bzw. Balintgruppen teil; fast alle empfanden dies als hilfreich. Ein knappes Drittel der Befragten hat sich schon einmal eine ambulante Ethikberatung gewünscht, 32\% waren sich unsicher, 37\% haben sich bisher keine Ethikberatung gewünscht. Über die Hälfte der Teilnehmer empfand die Etablierung einer Ethikberatung sinnvoll, ein gutes Drittel war sich nicht sicher, 11\% der Befragten empfanden die Etablierung als nicht sinnvoll. Ethikberatungen sollten vor allem telefonisch erfolgen; Beratungen in der Praxis oder am Krankenbett wurden selten gewünscht. Beratend tätig sein sollten vorwiegend Ärzte, besonders Hausärzte. Ambulante Pflegekräfte in Ethikkomitees wurden vergleichsweise selten gewünscht. Berater sollten über medizinisches Fachwissen und Erfahrungen in der ambulanten Patientenversorgung verfügen und sich im Medizinrecht auskennen. Eine Vergütung für die Inanspruchnahme einer Ethikberatung hielt ein Viertel für erforderlich.

\subsection{Stärken und Grenzen der Untersuchung}

Für die Datenerhebung boten sich verschiedene Möglichkeiten an: die schriftliche, die persönliche oder die telefonische Befragung. Aus folgenden Gründen erschien die schriftliche Befragung für diese Studie zweckmäßig:

Im Gegensatz zur persönlichen Befragung stellt die Fragebogenmethode ein sehr ökonomisches, d.h. einfach durchzuführendes und gut auszuwertendes Verfahren dar. Sie erfordert in der Regel einen geringen organisatorischen Aufwand (Porst 1998). Die Fragebogenmethode ist zudem weniger zeitintensiv als eine persönliche oder telefonische Befragung, wodurch ein größerer Adressatenkreis erreicht werden kann. Die Teilnehmer können ihre Antworten in aller Ruhe abwägen und Antworten gegebenenfalls korrigieren (Klein und Porst 2000), außerdem können die Zielpersonen selber entscheiden, wann sie den Fragebogen ausfüllen wollen (Porst 1998). Zudem können Teilnehmer die Beantwortung des Fragebogens unterbrechen, nachdenken und wenn gewünscht in ihren Unterlagen nachlesen. Sowohl die „Selbstbestimmtheit als auch die Anonymität der Befragungssituation führen dazu, dass die Antworten bei der postalischen Befragung als ,ehrlicher' gelten, als ,überlegter' und ,durchdachter'“ (Porst 1998, S. 16). 
Eine Schwäche der Fragebogenmethode ist die hohe Non-response Rate. Vor allem Hausärzte beteiligen sich ungern an Studien (Hummers-Pradier et al. 2008). Zudem bleibt unklar, wer den Fragebogen ausfüllt (Schnell et al. 1998) und in welcher Atmosphäre dies geschieht, zum Beispiel im Arbeitsumfeld oder „in einer Kneipe nach dem vierten oder fünften Bier“ (Jacob und Eirmbter 1999, S. 139). Nachfragen der Teilnehmer bezüglich unklarer Sachverhalte können nicht unmittelbar beantwortet werden. Bei der Entwicklung des Fragebogens wurde deshalb darauf geachtet, möglichst verständliches Vokabular zu nutzen. Eine weitere Schwäche stellt die „Ja-Sage-Tendenz“ dar, also das Bestreben der Befragten, den Fragen eher zuzustimmen. Um diese Anfälligkeit zu mildern, wurde geeigneten Fragen die Antwortmöglichkeit „bin mir nicht sicher“ zugefügt. Es muss weiterhin damit gerechnet werden, dass die Befragten tendenziell sozial erwünschte Antworten geben (Bortz und Döring 2015, Schnell et al. 1989). Zwar wird diesem Effekt in postalischen Befragungen eine untergeordnete Rolle beigemessen (Klein und Porst 2000); dennoch wurde den Teilnehmern der Studie explizit Anonymität zugesichert (siehe Anlage 9.1).

Neben diesen formalen Schwächen der Untersuchung muss die generelle Frage gestellt werden, inwieweit die postalische Befragungsmethode die Qualität von ethischen Konflikten ermitteln kann. Denn auch und vielleicht gerade weil Konflikte nicht durch ihre Häufigkeit imponieren, können sie den betroffenen Arzt erheblich belasten. Eine tiefere Exploration ethischer Konflikte ist sicherlich nicht möglich, wurde im Rahmen der Studie aber auch nicht als Ziel formuliert. Auch Häufigkeiten ethischer Konflikte können mit dieser Methode nur annähernd erfasst werden. Der Sinn dieser Studie lag allerdings explizit darin, eine erste orientierende Kenntnis über die Häufigkeit von ausgewählten Konflikten und den Wunsch nach einer ambulanten Ethikberatung zu erlangen. Hierzu liegen bisher keine Daten vor. Die Ausgestaltung von Ethikberatungen könnte demnach von dem Studienergebnis profitieren.

Nicht auszuschließen ist, dass sich vor allem Ärzte an der Umfrage beteiligen, die an der Errichtung einer Ethikberatung interessiert sind; die Meinung anderer wäre dadurch unterrepräsentiert. Um auch deren Meinung abbilden zu können, wurde dem zweiten Anschreiben ein Kurzfragebogen hinzugefügt, auf dem die Teilnehmer ausdrücklich angeben konnten: Ich würde keine Ethikberatung nutzen (siehe Anlage 9.5).

Am Hauptfragebogen könnte Folgendes kritisiert werden: In Frage 1 sollten die Teilnehmer die Häufigkeit von Entscheidungskonflikten benennen. Es bestand die Möglichkeit, dass manche Ärzte ein angegebenes Szenario nicht als Konflikt wahrnehmen. Durch eine weitere Spalte „empfinde ich nicht als konflikthaft“ hätten die Ärzte dem Ausdruck verleihen können.

Für die Berechnung der Stichprobe wurde angenommen, dass der Konflikt „Nachträgliches Umsetzten einer Patientenverfügung, wenn dieser beispielsweise in einer Notfallsituation nicht nachgekommen wurde“ von $70 \%$ der Befragten ca. einmal im Vierteljahr erlebt wird. 
Diese Einschätzung hat sich als nicht zutreffend erwiesen, nur 7,5\% erlebten diesen Konflikt ca. einmal im Vierteljahr oder häufiger (siehe Tabelle 3). Dieses Ergebnis verdeutlicht die Relevanz von Umfragen, da eine Diskrepanz zwischen wissenschaftlicher Einschätzung und Realität bestehen kann.

\subsection{Stichprobe}

Das Alter der Studienteilnehmer betrug durchschnittlich 54,0 Jahre und repräsentiert damit das Durchschnittsalter der deutschen Hausärzte bundesweit von 54,6 Jahren im Jahr 2014 (Kassenärztliche Bundesvereinigung 2015). Knapp 40\% der Studienteilnehmer waren weiblich, bundesweit praktizierten 42\% Hausärztinnen (Kassenärztliche Bundesvereinigung 2015).

Die Ausschöpfung der Stichprobe lag bei zusammengefasst 57,5\% (45,6\% Hauptfragebogen $+11,9 \%$ Kurzfragebogen). Dies ist ein vergleichsweise gutes Ergebnis, normal gilt eine Rücklaufquote von $40 \%$ bei postalischen Befragungen (Shih und Xitao Fan 2008), andere Untersuchungen gehen von einer Rücklaufquote zwischen $30-40 \%$ in der allgemeinen Bevölkerung aus (Jacob und Eirmbter 1999). Große Meinungsforschungsinstitute haben zum Teil eine Ausschöpfungsquote von unter 20\% (Rabe 2016). Die Zielgruppe der Hausärzte erachten Studien im Arbeitsfeld der Allgemeinmedizin generell als sinnvoll, ein großer Teil möchte aber selber nicht daran mitwirken (Hummers-Pradier et al. 2008). Die hohe Beteiligung an der vorliegenden Studie zeigt das große Interesse und damit die Relevanz einer ambulanten Ethikberatung.

\subsection{Konflikte von Hausärzten}

Umfragen an Krankenhäusern ergaben, dass ethische Konflikte vor allem die Beziehung von Ärzten mit Patienten und Angehörigen betreffen. Weitere Konfliktfelder betreffen das Lebensende von Patienten, Wahrheitsmitteilung, Patientenautonomie und Ressourcenrationierung (Aleksandrova 2008, DuVal et al. 2004, DuVal et al. 2001). Über die Art und die Häufigkeit von Konflikten in Hausarztpraxen ist bisher wenig bekannt. Das Institut für Allgemeinmedizin der Universitätsmedizin Göttingen hat in Fokusgruppen mit Hausärzten ethisch konflikthafte Situationen herausgearbeitet; die Mehrheit der Ärzte erlebten ethische Konflikte im diagnostischen und therapeutischen Vorgehen (Heßling et al. 2014). In der vorliegenden Studie wurden diese und weitere Konflikte in ihrer Quantität untersucht. Manche der abgefragten Konflikte, wie z. B. das Vorgehen bei strafrechtlich relevanten Informationen über Dritte, wurde von der Mehrheit der Ärzte eher selten erlebt. Die Angabe ,selten' lässt dabei keinen Rückschluss auf die Qualität und Bedeutung des Konfliktes für den Hausarzt zu. Ein Konflikt kann eine hohe Belastung für den betroffenen Arzt darstellen, auch wenn er nicht häufig er- 
lebt wird. Vor einer Interpretation ,selten und deshalb unbedeutend' muss daher gewarnt werden, selten erlebte Konflikte sind nicht zwangsläufig weniger belastend als häufig erlebte.

Fortsetzung einer potentiell kurativen Therapie, die schlecht vertragen wird. Nebenwirkungen von Medikamenten sind keine Seltenheit. Ohne Nebenwirkung keine Hauptwirkung, so heißt es in der Pharmakologie. Diese Aussage spiegelt die Problematik, der sich sowohl Patienten als auch behandelnde Ärzte häufig stellen müssen, prototypisch beim Morbus Parkinson. Als Folge dieser Erkrankung kommt es zu Muskelstarre, verlangsamten Bewegungen, Muskelzittern und Haltungsinstabilität. Standardmedikamente wie NonergolinDopaminagonisten haben Schläfrigkeit und Schlafattacken als Nebenwirkungen. Patienten müssen unter anderem darauf hingewiesen werden, kein Kraftfahrzeug zu führen (Möller et al. 2000). Doch was ist zu tun, wenn der Patient nicht auf das Autofahren verzichten möchte? Wenn er von Berufs wegen auf das Führen eines Fahrzeuges angewiesen ist? Die Entscheidung, sich trotz starker Nebenwirkungen zwischen der Weiterführung einer Therapie, deren Umstellung oder einem Abbruch zu entscheiden, ist für Hausärzte konfliktreich - ein Großteil der Befragten sah sich ca. einmal im Monat mit einer vergleichbaren Problematik konfrontiert, Ärzte eher als Ärztinnen.

Fortsetzung einer potentiell lebensverlängernden Therapie bei infauster Prognose, die schlecht vertragen wird. Die scheinbar unbegrenzten Möglichkeiten in der Intensivmedizin zum Erhalt des Lebens in Kombination mit dem Lebenserhaltungsprinzip können Ärzte vor ethische Herausforderungen stellen. Was ist zu tun, wenn die Therapie einer Erkrankung schlecht vertragen wird, sie aber ggf. das Leben des Patienten rettet? Die Bundesärztekammer positioniert sich bei diesen Patienten folgendermaßen: Bei Patienten mit infauster Prognose, die sich aber noch nicht im Sterben befinden, kommt eine Änderung des Behandlungszieles nur dann in Betracht, wenn die Krankheit weit fortgeschritten ist und eine lebenserhaltende Behandlung nur Leiden verlängert (Bundesärztekammer (2011). Patienten sollten von den behandelnden Ärzten vor einer Übertherapie am Lebensende geschützt werden (Nauck 2011). Doch wann hat ein unumkehrbarer Sterbeprozess begonnen hat und wann kann noch kurativ behandelt werden? Dieser Grenzbereich ist häufig nicht zu erkennen (Nauck 2011). „Die Beurteilung, ob der Sterbeprozess eines Menschen bereits begonnen hat und ob Maßnahmen eine Verlängerung des Sterbens oder des Lebens bedeuten würden“ (Nauck 2011, S. 140), ist nicht nur auf Intensivstationen häufig schwierig. Eine Auflistung von Ethikkonsilen an der Universität Freiburg benennt „Indikationen zur Therapiebegrenzung“ als weitaus häufigsten Grund einer Ethikberatung: Von Juli 2001 bis Juli 2002 war dieser Konflikt 21-mal Gegenstand einer Ethikberatung (Maio 2002). Auch die befragten Hausärzte sahen sich - trotz der Stellungnahme der Bundesärztekammer - durchaus mit diesem Konflikt kon- 
frontiert; knapp 20\% 1-mal pro Monat oder häufiger, 23\% ca. einmal alle 3 Monate und ein Viertel ca. einmal alle 6 Monate.

Fortsetzung einer Therapie, wenn der Patientenwille wegen einer progredienten Erkrankung nicht mehr feststellbar ist. Ärzte sollen dem Patienten (1) nicht schaden, sondern (2) ihm nutzen und (3) seinen Willen berücksichtigen. Der (4) Wille von Angehörigen soll mitberücksichtigt werden, und es soll (5) das effizienteste Kosten-Nutzen-Verhältnis angewendet werden (Synofzik und Maetzler 2007). Wenn der Patientenwille zum Beispiel in Folge einer Demenz oder eines schweren Traumas nicht mehr zu ermitteln ist, kann die Fortsetzung einer Therapie - besonders in Bezug auf die Berücksichtigung des Patientenwillens - zu Konflikten führen. „Denn auch und gerade Demenz-Patienten haben ein Recht, ihre Krankheit zu haben und zu leben“ (Synofzik und Maetzler 2007, S. 276). Der Bundesgerichtshof kommentiert:

„Sind die Vitalfunktionen des Patienten nicht beeinträchtigt, steht sein Tod nicht unmittelbar bevor, kommt es für die Fortsetzung oder Beendigung der Behandlung auf den aktuellen Willen des Patienten an, hilfsweise, wenn er sich nicht mehr aktuell äußern kann, auf den antizipativen Willen (zum Beispiel in Gestalt einer Patientenverfügung oder - subsidiär - auf den mutmaßlichen Willen. Lässt sich weder ein antizipativer noch mutmaßlicher Wille feststellen, sollen nach Ansicht des BHG (in Strafsachen) die allgemeinen Wertvorstellungen“ entscheiden, wobei „jedoch Zurückhaltung geboten“ und „im Zweifel dem Schutzmenschlichen Lebens Vorrang vor persönlichen Überlegungen des Arztes, der Angehörigen oder einer anderen Beteiligten Person" einzuräumen ist" (Ulsenheimer 2009, S. 726).

Mehr als 40\% der befragten Hausärzte gaben an, diesen Konflikt seltener als alle 6 Monate zu erleben, $12 \%$ hatten diesen Konflikt noch nicht erlebt.

\section{Informationsweitergabe an Angehörige, wenn nicht bekannt ist, ob der Patient dies} gestattet hat. Als historische Grundlage der Schweigepflicht wird der hippokratische Eid angesehen, in dem es heißt: „Über alles, was ich während oder außerhalb der Behandlung im Leben der Menschen sehe oder höre und das man nicht nach draußen tragen darf, werde ich schweigen und es geheim halten“ (Bauer 1993, S. 2). Im Jahr 1948 wurde vom Weltärztebund eine Art Neufassung des hippokratischen Eides verabschiedet, das Genfer Gelöbnis: „Ich werde alle mir anvertrauten Geheimnisse auch über den Tod des Patienten hinaus wahren" (Ziegler 2007, S. 43). Das Bundesverfassungsgericht betonte den Vertrauensschutz im Jahr 1972 durch folgende Stellungnahme:

„Wer sich in ärztliche Behandlung begibt, muss und darf erwarten, dass alles, was der Arzt im Rahmen seiner Berufsausübung über seine gesundheitliche Verfassung erfährt, geheim bleibt und nicht zur Kenntnis Unberufener gelangt. Nur so kann zwischen Patient und Arzt jenes Vertrauen entstehen, das zu den Grundvoraussetzungen ärztlichen Wirkens zählt (...) “ (Ulsenheimer 2014, S. 529).

Jeder Arzt unterliegt der Schweigepflicht. Ein erfolgreiches ärztliches Handeln wäre ohne sie nicht möglich (Ziegler 2007). Der Patient soll seinem Arzt „alle Probleme und Nöte mitteilen 
können, ohne befürchten zu müssen, dass diese an Dritte oder die Öffentlichkeit geraten oder sogar Grundlage möglicher Sanktionen werden“ (Frewer und Säfken 2003, S. 15). Die ärztliche Schweigepflicht gilt auch bei schriftlichen Mitteilungen des Patienten (Ziegler 2007) und postmortal (Roebel et al. 2009). Sie ist ein hoch geschütztes Gut, darf aber nicht um jeden Preis aufrecht erhalten werden (Parzeller et al. 2005). Besonders problematisch sind dabei Fälle, in denen Dritte durch das ärztliche Schweigen gesundheitlicher Gefahren ausgesetzt sind (Frewer und Säfken 2003).

Unsicherheiten hinsichtlich der Rechtslage und die Sorge vor Konsequenzen begleiten regelmäßig die ärztliche Entscheidungsfindung (Kemper et al. 2010). Folgendes Beispiel soll dies veranschaulichen: Im Jahr 1993 informiert ein Patient seinen Hausarzt über seine AIDSErkrankung und verbietet ausdrücklich eine Weitergabe dieser Information an Dritte, eingeschlossen seiner Lebensgefährtin. Diese lässt sich später von demselben Arzt behandeln, er verschweigt ihr die Erkrankung ihres Partners. Ein Jahr später verstirbt der Patient, eine Blutuntersuchung der Ehefrau ergibt eine HIV-Infektion. Sie verklagt ihren Arzt auf Schmerzensgeld in Höhe von 100.000 DM (Frewer und Säfken 2003). Ähnliche Konflikte wurden von den befragten Hausärzten vergleichsweise selten erlebt. Über die Hälfte der Befragten gab an, seltener als einmal in 6 Monaten oder bisher noch nicht mit dieser Problematik konfrontiert gewesen zu sein. Möglicherweise treten Konflikte bezüglich der ärztlichen Schweigepflicht vor allem im Krankenhaus auf: die Patienten dort sind schwerkrank, eine Eigenanamnese ist häufig nicht möglich. Informationen über den Patienten müssen dann von Angehörigen eingeholt werden, die in der Folge auch über den Zustand ihres Angehörigen informiert werden möchten. Die Verpflichtung zur Verschwiegenheit kann dann oft nur schwer eingehalten werden (Ziegler 2007). Hausärzte arbeiten in einem anderen Umfeld, die dargestellte Problematik stellt sich innen weit seltener oder anders. Zu gerichtlichen Auseinandersetzung und Sanktionen bei unbefugtem Bruch der Schweigepflicht kommt es insgesamt selten (Roebel et al. 2009), möglicherweise spielt der Konflikt auch deshalb eine eher untergeordnete Rolle.

\section{Nachträgliches Umsetzen einer Patientenverfügung, die in einer Notfallsituation nicht} berücksichtigt wurde. Immerhin 75\% der älteren Bevölkerung haben keine Patientenverfügung (Harringer et al. 2009), wodurch mitunter Konflikte zwischen Angehörigen und Ärzten entstehen können (Marti und Bovet 2004). Ein anschauliches Beispiel ist der Fall Terri Schiavo aus Florida. Eine junge Frau fällt ins Koma, ihr Ehemann und ihre Eltern sind sich bezüglich der künstlichen Ernährung nicht einig. 15 Jahre später erlaubt ein Gericht die Einstellung der Nahrung, Terri Schiavo stirbt (Frankfurter Allgemeine Zeitung 2005). In diesem Fall konnte die Betroffene nicht mehr nach ihrem Willen befragt werden. Den Konflikt des nachträglichen Umsetzens einer Patientenverfügung erlebte gut ein Drittel der Hausärzte seltener 
als alle 6 Monate; knapp die Hälfte hat diesen Konflikt bisher nicht erlebt. Die Vermutung, dass ca. $70 \%$ der Ärzte diesen Konflikt mindestens einmal im Vierteljahr erleben, kann nicht bestätigt werden. Das mag daran liegen, dass sich - ähnlich dem vorherigen Konflikt - im Krankenhaus tätige Ärzte und Notärzte eher mit dieser Thematik konfrontiert sehen, da betroffene Patienten von Notärzten primär in ein Krankenhaus und nicht zu einem Hausarzt transportiert werden.

\section{Einweisung von pflegebedürftigen oder wesensveränderten Patienten in Pflegeeinrich-} tungen gegen deren Willen. Viele Menschen wünschen sich, in ihrer vertrauten Umgebung zu sterben; diesem Wunsch kann aufgrund verschiedener Einflussfaktoren häufig nicht entsprochen werden. Vor allem Hausärzte und Verwandte des Patienten nehmen hier Einfluss auf die Erfüllung dieses Wunsches (Gágyor et al. 2016). Weitere Situationen, in denen die ärztliche Einweisung eines Patienten gegen seinen Willen sinnvoll sein kann und zu erwägen ist, sind psychiatrische Erkrankungen, Demenz oder anderen Erkrankungen, in deren Folge der Patient sich selber oder Dritte gefährdet. Die freiheitlichen Persönlichkeitsrechte sind ein hohes Gut und werden durch den Gesetzgeber unter besonderen Schutz gestellt (Müller 2004), für eine Einweisung gegen den Willen eines Patienten ist die Kenntnis der rechtlichen Situation erforderlich (Prüter 2008). Schon 1977 positioniert sich die World Psychiatric Association folgendermaßen:

\footnotetext{
„No treatment should be provided against the patient's will, unless withholding treatment would endanger the life of the patient and/or those who surround him or her. Treatment must always be in the best interest of the patient" (Steinert et al. 2001, S. 700).
}

Trotz gesetzlicher Regelung und ethischer Leitlinien verbleibt häufig ein beträchtlicher Unsicherheitsbereich (Steinert et al. 2001). Knapp die Hälfte der befragten Hausärzte sah sich mit diesem Konflikt seltener als alle sechs Monate konfrontiert, weitere 15\% hatten diesen Konflikt bisher nicht erlebt. Möglicherweise wird die Einweisung psychisch kranker Menschen in akuten Fällen eher durch einen Notarzt (Prüter 2008) oder in weniger akuten Fällen durch den schon behandelnden Psychiater vorgenommen, so dass Hausärzte weniger häufig mit solchen Konflikten konfrontiert werden. Diese Einschätzung bestätigt eine Schweizer-Studie, in der untersucht wurde, ob Zeugnisse der Fürsorgerischen Freiheitsentziehung (FFE) durch unterschiedliche Facharztgruppen formal und inhaltlich korrekt ausgefüllt wurden. Psychiater schneiden in dieser Untersuchung am besten ab, Hausärzte am schlechtesten, da sie in diesem Gebiet sicherlich am wenigsten Erfahrung haben, „wenige stellen wohl häufiger als ein bis zwei Mal pro Jahr ein FFE-Zeugnis aus“ (Maier 2001, S. 1581).

Selbstgefährdendes Patientenverhalten. Die Themen Krankheit und Gesundheitsverhalten sind in den letzten Jahren vermehrt in die Öffentlichkeit gerückt und mit innen der Begriff der Medikalisierung (Schneider 2013). Unter dem Begriff der Medikalisierung versteht man 
unter anderem, „dass die Medizin sowie das gesamte System der Gesundheitsversorgung mehr und mehr den Alltag und gesellschaftliche Bereiche bestimmen" (Schneider 2013, S. 220). Diese Entwicklung wird durch verschiedene Komponenten vorangetrieben: Zum einen werden neue Krankheiten geschaffen oder Krankheitsbegriffe ausgeweitet, zum anderen werden „normale“ Zustände zu behandlungsbedürftigen erklärt (Schneider 2013). Auch Lifestyle-Medikamente, eine Medikamentengruppe, beispielsweise für Haarwachstum, Gewichtsreduktion oder andere kosmetische Fragestellungen, die dem Bedarf der westlichen Gesellschaft nach Schönheitsidealen entsprechen (Harth et al. 2003), tragen zur Medikalisierung bei (Schneider 2013). Eine zentrale Rolle in dieser Entwicklung nehmen, neben Ärzten, Patienten ein: Der Arzt-Patienten-Kontakt erfolgt seit Einführung von Lifestyle-Medikamenten vermehrt in der klaren Absicht einer definierten Wunschmedikation, häufig ohne Therapiegrundlage (Harth et al. 2003). Ärzte kommen diesem Wunsch oftmals nach. Eine amerikanische Studie zeigte, dass Patienten, die ein bestimmtes Medikament verlangten, dieses häufiger verschrieben bekamen als Patienten, die nach keinem Mittel fragten (Kravitz et al. 2005). Den Konflikt des selbstgefährdenden Patientenverhaltens nahmen auch die befragten Hausärzte wahr: jeweils über $20 \%$ sahen sich ca. einmal alle 6 Monate oder ca. einmal alle 3 Monate mit selbstgefährdendem Patientenverhalten konfrontiert, über 15\% nahmen diese Thematik ca. einmal im Monat oder öfter wahr, Männer häufiger als Frauen.

\section{Vorgehen bei strafrechtlich relevanten Informationen über Dritte (beispielsweise häus-} liche Gewalt durch den Partner). Häusliche Gewalt ist nach Angaben der WHO eines der weltweit größten Gesundheitsrisiken für Frauen und Kinder. Unter häuslicher Gewalt versteht man eine Gewaltausübung zwischen Personen, die in einer engen sozialen Beziehung stehen. Dabei beschränkt sich dieser soziale Nahraum nicht nur auf aktuelle oder ehemalige Lebenspartner, sondern schließt auch Mitglieder der Familie, Angehörige und Freunde mit ein (Gologan et al. 2014). Eine Studie an einem Krankenhaus der Maximalversorgung kommt zu dem Ergebnis, dass 70\% der Opfer häuslicher Gewalt von Hausärzten weiter versorgt werden (Gologan et al. 2014). Die verschiedenen zu beachtenden Gesetze, verursachen neben hohem Handlungsdruck auch Handlungsunsicherheiten (Kemper et al. 2010). Betroffene Patienten werden von Hausärzten (weiter-)versorgt, dennoch wurde der Konflikt des weiteren Vorgehens bei strafrechtlich relevanten Informationen über Dritte von mehr als der Hälfte der Befragten seltener als alle sechs Monate erlebt, ein Viertel hat diesen Konflikt bisher nicht erlebt, Männer erlebten inn häufiger als Frauen. Möglicherweise wird das weitere Vorgehen - wenn nötig - bereits von dem im Krankenhaus tätigen Arzt geplant. 


\section{Konträre Behandlungsansichten zwischen mir und einer stationären Einrichtung.}

Fast jeder Patient erfährt nach einem Krankenhausaufenthalt eine Medikamentenänderung (Himmel et al. 2004); so werden in manchen Fällen sterbenden Patienten im Krankenhaus Lipidsenker zur Entfernung schädlicher Fette aus dem Blutkreislauf verschrieben, obwohl die Lebensqualität von Patienten höher ist, die keine Statine bekommen (Kutner et al. 2015). Divergierende Meinungen zwischen dem weiterbehandelnden Hausarzt und dem im Krankenhaus tätigen Arzt können die Folge von Medikamentenveränderungen sein. Eine Studie zeigt, dass es für mehr als die Hälfte der im Krankenhaus verschriebenen Protonenpumpeninhibitoren keine adäquate Indikation gibt (Ahrens et al. 2012). Die Entscheidung, ob Hausärzte ihren Patienten empfehlen, die vorgeschlagene Medikation ambulant fortzuführen, hängt unter anderem von dem Verhältnis zwischen dem im Krankenhaus tätigen Arzt und dem Hausarzt ab (Wermeling et al. 2014). Die Beziehung zwischen im Krankenhaus tätigen Ärzten und Hausärzten kann sich somit auch auf die Patientenversorgung auswirken. $\mathrm{Zu}$ diesem Ergebnis kommen auch Sampson et al. 2016. Missverständnisse resultieren unter anderem durch eine aufwändige Kommunikation: Sowohl für Hausärzte als auch für im Krankenhaus tätige Ärzte, ist der telefonische Kontakt mit ihrem Kollegen mühsam und frustrierend; wenn ein Gespräch zustande kommt, so verhindert Zeitnot häufig einen informativen Austausch (Sampson et al. 2016). Den im Krankenhaus tätigen Ärzten fehlen häufig Informationen des Hausarztes bezüglich der aktuellen emotionalen Verfassung des Patienten. Sowohl im Krankenhaus tätige Ärzte als auch Hausärzte bemängeln die Delegation von Arbeit in den jeweils anderen Bereich. Konträre Behandlungsansichten zwischen Hausärzten und einer stationären Einrichtung nahmen auch über 20\% der Befragten unserer Studie einmal pro Monat oder öfter wahr, ein Viertel erlebte diesen Konflikt öfter als alle drei Monate.

Absetzen von Arzneien bei mittlerweile fraglichem Nutzen für den Patienten. Ein erheblicher Anteil der älteren Bevölkerung nimmt mehrere unterschiedliche Medikamente gleichzeitig ein. Wenn Patienten täglich fünf oder mehr unterschiedliche Medikamente einnehmen, spricht man von Polypharmazie. Die Furcht vieler Ärzte, durch das Absetzen von Medikamenten ein unerwünschtes Ereignis hervorzurufen, ist verständlicherweise hoch (Thürmann 2013). Diese Studie bestätigt die Relevanz dieses Konfliktes; über die Hälfte aller Teilnehmer erlebte inn ca. einmal pro Monat. Eine Beschäftigung der Hausärzte mit dieser Thematik scheint notwendig, da sich durch das Absetzen von Medikamenten das Wohlbefinden und die kognitiven Leistungen von Patienten verbessern können (Garfinkel und Mangin 2010). 


\section{Sinnhaftigkeit diagnostischer Maßnahmen zur Früherkennung bei bereits schwerkran-}

ken Patienten. Wenn ein Patient schwer erkrankt, stellt sich die Frage, inwieweit ScreeningMaßnahmen noch sinnvoll sind. Was nützt zum Beispiel einem Patienten, der eventuell bereits kurz vor seinem Tod steht, die Information, dass er neben einer schweren Erkrankung zusätzlich eine Vergrößerung seiner Prostata entwickelt hat? Studien haben ergeben, dass sich terminal erkrankte Patienten am stärksten vor Schmerzen fürchten. Außerdem fürchten sich Patienten neben einem Autonomieverlust davor, anderen zur Last zu fallen (Magon 2014); die Angst vor einer weiteren Erkrankung wird in diesem Zusammenhang nicht genannt. Für im Krankenhaus tätige Ärzte spielt dieser Konflikt eine Rolle (Reiter-Theil 2000). Durch die vorliegende Studie ist deutlich geworden, dass er auch in der hausärztlichen Versorgung eine bedeutende Rolle einnimmt: ein Drittel der befragten Hausärzte erlebte diesen Konflikt ca. einmal im Monat, weitere $30 \%$ ca. einmal alle drei Monate, Ärzte erlebten inn häufiger als Ärztinnen.

\section{Patienten und Angehörigen sind uneins bzgl. diagnostischer bzw. therapeutischer} Maßnahmen. Die Verweildauer von Patienten in Krankenhäusern hat sich in den letzten Jahren verkürzt (Handel 2003). Parallel zu dieser Entwicklung steigt die Zahl der Demenzerkrankten, von denen viele Patienten zu Hause leben (Handel 2003). Beide Entwicklungen bewirken, dass die Pflege und Betreuung von Patienten zunehmend in den Verantwortungsbereich ihrer Angehörigen verlagert wird. Diese neue Rollenverteilung kann schwierig und konfliktgeladen sein (Handel 2003). Neben der körperlichen Pflege erleben die Angehörigen auch hautnah die sozialen und seelischen Probleme der Kranken bzw. sind selbst davon betroffen. Die pflegenden Angehörigen erleiden einen Verlust an Autonomie, zum Beispiel in der freien Zeiteinteilung, und sind zudem finanziell belastet. Bis zu zwei Drittel der pflegenden Angehörigen leiden dadurch an gesundheitlichen Problemen (Handel 2003). Der Schluss liegt nahe, sich mit dieser Problematik an den Hausarzt zu wenden. Ein Konflikt kann schnell erwachsen, wenn der Hausarzt neben dem pflegenden Angehörigen auch den betroffenen Patienten betreut.

Die Mehrheit der befragten Hausärzte erlebte diesen Konflikt ca. einmal alle drei Monate. Der Konflikt zwischen verschiedenen Parteien wird aber auch von anderen Fachärzten als ethisches Dilemma empfunden (DuVal et al. 2004, DuVal et al. 2001). Möglichkeiten zur Entschärfung des Konfliktes könnten unter anderem die Verbesserung von Unterstützungsmöglichkeiten von Angehörigen durch Beratung und deren Gesundheitsförderung sein sowie in der Entwicklung von zukünftigen Wohn- und Betreuungsformen kranker Menschen (Handel 2003). 


\subsection{Teilnahme an Qualitätszirkeln, Supervisions- und Balintgruppen und deren Unterstützung bei der Lösung von Konflikten}

Qualitätszirkel sowie Supervisions- und Balintgruppen haben sich im ärztlichen Alltag etabliert. So sucht eine Mehrheit der Ärzte in der vorliegenden Studie Unterstützung in Qualitätszirkeln und Balintgruppen. Qualitätszirkel verfolgen generell diverse Ziele (siehe 4.2), während in Balintgruppen typischerweise Konflikte in der Arzt-Patienten-Beziehung bearbeitet werden. Das ist zunächst ein Selektionsphänomen: nur eine kleine Gruppe von Hausärzten nimmt an Balintgruppen teil - diese Gruppe fühlte sich dann auch bei konflikthaften Situationen durch Supervisions- und Balintgruppen zumeist gut unterstützt (85,3\%); etwas seltener wurde dies von Qualitätszirkeln (54,7\%) berichtet. Das Ergebnis bestätigt in jedem Fall die Relevanz dieser Institutionen. Die Vermutung, Supervisions- und Balintgruppen helfen bei Lösung von Konflikten, kann bestätigt werden. Für Qualitätszirkel gilt diese Aussage mit Einschränkungen.

\subsection{Wunsch von Hausärzten nach einer Ethikberatung}

Fast ein Drittel aller Befragten hat sich bereits eine Ethikberatung gewünscht, etwa ein Drittel war sich unsicher, ein weiteres Drittel wünschte sich keine Ethikberatung. Die Skepsis von Kritikern einer klinischen Ethikberatung (siehe 2.6.2), klinische Ethikberatung sei von Ärzten nicht akzeptiert, kann bei diesem Antwortverhalten nur eingeschränkt auf eine ambulante Ethikberatung übertragen werden.

Tendenziell wünschten sich eher junge Ärzte eine ambulante Ethikberatung, Ärztinnen eher als Ärzte, obwohl einige Konflikte von Ärzten häufiger empfunden wurden als von Ärztinnen (siehe 6.3). Dies könnte man als Beleg für Geschlechterstereotype sehen: Während Frauen eher expressive Eigenschaften bzw., wie es Abele (2003) nennt, communal-expressiv traits, zugeschrieben werden (einfühlsam, hilfsbereit, freundlich), verfügen Männer eher über agentic-instrumental traits (durchsetzungsfähig, entscheidungsfreudig, selbstsicher) (Abele 2003). Diese Beobachtungen scheinen sich auch in der vorliegenden Studie zu bestätigen: Frauen erleben Konflikte weniger häufig, da sie durch ihre Einfühlsamkeit einem Konflikt vorbeugen, während Männer mit der ihnen zugeschriebenen Durchsetzungsfähigkeit einen Konflikt entscheiden - eine ambulante Ethikberatung ist für sie dadurch weniger häufig erforderlich. Einen Geschlechterunterschied in dem Wunsch nach einer Ethikberatung stellte auch eine Studie an der Medizinischen Universität Pleven in Bulgarien fest. Zum Zeitpunkt der Befragung gab es dort keine Möglichkeit einer ethischen Beratung. Die große Mehrheit der befragten Ärzte hat sich in ihrer bisherigen Laufbahn eine Ethikberatung gewünscht, Ärztinnen signifikant häufiger als Ärzte (Aleksandrova 2008). 
Häufiger wünschten sich jüngere Ärzte eine Unterstützung durch eine Ethikberatung. Für junge Ärzte, die von dem Setting eines Krankenhauses in das einer Hausarztpraxis wechseln, bestehen Konflikte nicht selten in der Konfrontation mit Alltagsproblemen, die innen oft noch nicht ausreichend vertraut sind. Während der Allgemeinarzt acht von zehn Patienten mit Herzproblemen wieder in die Häuslichkeit entlässt und dadurch viele Erfahrungen im Laufe seines Berufslebens sammelt, ist dies für im Krankenhaus tätige Ärzte ein eher unbekanntes Terrain (Donner-Banzhoff 2008). Möglicherweise fühlen sich länger praktizierende Ärzte eher in der Lage, Konflikte alleine zu lösen, während jüngere Ärzte aufgrund der geringeren Berufserfahrung noch zu selten mit solchen Konflikten konfrontiert wurden und nach Unterstützung suchen.

Die Vermutung, dass sich Ärzte aus Einzelpraxen eher eine Ethikberatung wünschen als ihre Kollegen aus Gemeinschaftspraxen/Praxisgemeinschaften, scheint nicht zuzutreffen. Fast $40 \%$ der in Einzelpraxen praktizierenden Ärzte wünschten sich bisher keine ambulante Ethikberatung. Für Ärzte in ethischen Konfliktsituationen ist das Gespräch mit Kollegen bei der Lösung des Problems am ehesten hilfreich (DuVal et al. 2004). Die Verantwortung bei schwierigen Entscheidungen zu teilen, entlastet Hausärzte (Donner-Banzhoff 2008). Insofern ist dieses Ergebnis überraschend. Eventuell sind Ärzte aus Einzelpraxen in ihrer Persönlichkeit von vornherein eher eigenständig und ungebunden, sie lösen ihre Konflikte alleine.

Fast ein Drittel der Teilnehmer war sich bei der Frage nach dem Wunsch nach einer Ethikberatung „nicht sicher“. Dies liegt wahrscheinlich an der noch fehlenden Bekanntheit eines ambulanten Ethikkomitees; vielleicht fürchten manche Ärzte aufgrund fehlender Informationen eine Mehrbelastung durch die Inanspruchnahme solcher Institutionen.

\subsection{Etablierung einer Ethikberatung}

Die Etablierung einer Ethikberatung empfand über die Hälfte als sinnvoll; wie schon bei dem Wunsch nach einer Ethikberatung waren es mehr Frauen und häufiger junge Ärzte. Eine Begründung für dieses unterschiedliche Antwortverhalten von Ärztinnen und Ärzten wurde bereits genannt (siehe 6.4). Ein ähnliches Ergebnis zeigt die erwähnte Studie an bulgarischen Ärzten: knapp 90\% empfand die Etablierung einer Ethikberatung als vorteilhaft (Aleksandrova 2008). Erwähnt werden muss in diesem Zusammenhang allerdings, dass die Antwortmöglichkeit „bin mir nicht sicher“ den Ärzten in der bulgarischen Untersuchung nicht zur Verfügung stand.

Das Alter der befragten Hausärzte spielte auch im Hinblick auf die Etablierung einer Ethikberatung eine Rolle: wie eingangs vermutet, waren ältere Ärzte an einer Etablierung seltener interessiert. Möglicherweise halten sie sich mit zunehmender Berufserfahrung kompetenter bei der Konfliktlösung. 
Dass sich nur etwa ein Drittel der befragten Hausärzte eine Ethikberatung wünschte, eine Etablierung aber über die Hälfte als sinnvoll erachteten, dürfte im ersten Moment verwundern. Möglicherweise war eine ambulante Ethikberatung für Hausärzte in der Vergangenheit keine Option, so dass sie sich mit dem Wunsch nach einer Beratung gar nicht erst auseinandergesetzt hatten. Auch das Bedürfnis nach Sicherheit könnte dieses Antwortverhalten erklären - sollte es in der weiteren Laufbahn einen Konflikt geben, der alleine nicht gelöst werden kann, könnte eine zuverlässige Anlaufstelle eine Erleichterung für Ärzte darstellen. Eine weitere Interpretation wäre, dass viele Ärzte sich selber in der Lage sehen, ihre Konflikte alleine zu lösen, ihren Kollegen aber eine Hilfestellung wünschen.

Knapp über die Hälfte der Hausärzte aus Einzelpraxen empfand die Etablierung einer Ethikberatung sinnvoll. Dies ist erwähnenswert, da ein großer Teil dieser Ärzte bisher nicht den Wunsch nach einer Ethikberatung hatte (siehe 6.5). Auch hier könnte das Bedürfnis nach Sicherheit eine Rolle spielen, da Ärzte aus Einzelpraxen im Ernstfall keine Kollegen in ihrer direkten Arbeitsumgebung um Hilfe bitten können. Die anfängliche Vermutung, dass Ärzte aus Einzelpraxen eine Etablierung eher sinnvoll finden, scheint zuzutreffen.

Über ein Drittel der Befragten war sich nicht sicher, ob die Etablierung einer Ethikberatung sinnvoll ist. Dies liegt möglicherweise daran - wie schon in 6.5 erwähnt - dass sich hinter dem Begriff der ambulanten Ethikberatung keine klare Definition verbirgt. Vielleicht können sich Hausärzte unter diesem Begriff wenig vorstellen und möchten sich vor einer Mehrbelastung schützen.

\subsection{Angebote, berufliche Hintergründe und Qualifikationen für Ethikberater}

Die Ärzte wünschten sich die Ethikberatung vorwiegend in Form einer telefonischen Beratung oder einer retrospektiven Fallbesprechung und wünschten sich Fortbildungen zu medizinethischen Fragestellungen. Beratungen in der Praxis oder am Krankenbett wurden vergleichweise selten gewünscht. Selbst für Pharmareferenten ist es aufgrund mangelnder Zeit des Arztes schwer geworden, einen Arzt zusehen (Wallenstein et al. 2006). Ein persönliches Treffen mit einem Ethikberater könnte abschreckend wirken; der Zeitpunkt für eine telefonische Beratung oder eine Fortbildung kann dagegen selber bestimmt werden.

Die Entwicklung von Leitlinien für wiederkehrende medizinische Konfliktsituationen wünschten sich nur etwa 40\% der Teilnehmer. Dabei können Leitlinien „durch eine bessere Kenntnis der Rechtslage und der medizinethischen Entscheidungsgrundlage zu einer größeren Handlungssicherheit im Umgang mit Entscheidungen mit Therapiezieländerungen führen“ (Jox et al. 2012, S. 829).

Die Mehrheit der Ärzte sprach sich für Hausärzte und Ärzte im Allgemeinen als Ethikberater aus. Es ist bekannt, dass Ärzte bei Konflikten in erster Linie Ratschläge von Kollegen einho- 
Ien (Aleksandrova 2008). Seelsorger in Ethikkomitees wünschten sich dagegen weniger als die Hälfte der Befragten. Seelsorge nimmt in Krankenhäusern, vor allem auf Palliativstationen eine wichtige Rolle ein: Durch die spirituelle Begleitung tragen Seelsorger zum Erhalt oder zur Verbesserung der Lebensqualität bei (Deutsche Gesellschaft für Palliativmedizin 2007). Ethik-Beauftragte christlicher Trägerorganisationen sollen ausdrücklich auf das christliche Menschenbild und die christliche Sozialethik geschult werden (May et al. 2010). Ein Grund für das eingeschränkte Interesse an Seelsorgern in ambulanten Ethikkomitees kann der fehlende religiöse Hintergrund von Hausärzten bzw. Hausarztpraxen sein. Während in Deutschland jedes dritte deutsche Allgemeinkrankenhaus einen konfessionellen Hintergrund besitzt (Christliche Krankenhäuser in Deutschland 2016) kann dieser Hintergrund bei ambulanten Praxen weitgehend ausgeschlossen werden. Pflegekräfte in Ethikkomitees wünschte sich nur ein Viertel der Befragten, mehr Frauen als Männer. Spannungen zwischen Pflegekräften und Ärzten sind im Krankenhaus keine Seltenheit (Hibbeler 2011), dieses Ergebnis überrascht demnach nicht. Ob der Geschlechtsunterschied im Antwortverhalten darin begründet liegen, dass häufiger Ärztinnen als Ärzte vor ihrem Studium eine Ausbildung in der Krankenpflege absolviert haben (Bundesagentur für Arbeit 2011) und dadurch besser über die Kompetenzen von Pflegekräften informiert sind, muss Spekulation bleiben.

Ethikberater sollten über medizinisches Fachwissen und Erfahrungen in der ambulanten $\mathrm{Pa}$ tientenversorgung verfügen. $\mathrm{Zu}$ einem ähnlichen Ergebnis kommt Aleksandrova: die klinische Kompetenz von Ethikberatern wurde am häufigsten als wünschenswerte Qualifikation genannt, gefolgt von den Fähigkeiten zur Konfliktlösung, Kommunikationsfähigkeit und Toleranz für verschiedene Sichtweisen (Aleksandrova 2008). In der vorliegenden Studie wünschte sich ein Großteil der Hausärzte, dass Ethikberater über Kenntnisse im Medizinrecht verfügen. Über Konflikte bezüglich des Medizinrechts finden sich in der Literatur unterschiedliche Angaben: Probleme im Medizinrecht empfanden in einer Umfrage von DuVal et al. 2001 nur 2\% der befragten Ärzte als ethisches Dilemma, während für Winkler et al. 2012 vor allem Entscheidungen für oder gegen lebensverlängernde Maßnahmen als juristische und als ethische Herausforderung gelten, insbesondere, wenn ein Austausch mit dem Patienten nicht mehr möglich ist.

\subsection{Vergütung}

Das Streben nach Gerechtigkeit ist in unserer Gesellschaft tief verankert, so auch das streben nach einer gerechten Vergütung (von Eisenhart Rothe 2015). Die berufliche Zufriedenheit von Ärzten steigt nicht zwangsläufig mit zunehmendem Gehalt (Beitzel et al. 2011); die Bezahlung sollte von den Ärzten vielmehr als ,fair' empfunden werden (Janus et al. 2009). Aufgaben, die nicht bezahlt werden, aber dennoch als sinnvoll erachtet werden, können zu 
einer Verringerung psychischer Probleme führen (Frese 1987) und werden daher in manchen Fällen von Arbeitnehmern erbracht, auch wenn diese Leistungen nicht vergütet werden. Möglicherweise zählt eine ambulante Ethikberatung hierzu: In unserer Befragung würde knapp die Hälfte (48\%) der Befragten eine ambulante Ethikberatung in Anspruch nehmen, auch wenn diese nicht vergütet werden würde. In der multivariaten Analyse spielte die Vergütung mit den Antwortmöglichkeiten „ja“ und „bin mir nicht sicher“ eine wichtige Rolle für den Wunsch nach einer Ethikberatung (siehe Tabelle 6), hier zeigt sich, dass sich eher die Ärzte eine Ethikberatung wünschten, die auch eine Vergütung für angebracht hielten.

\subsection{Kurzfragebogen}

Diejenigen Teilnehmer, die auf das zweite Anschreiben lediglich mit der Rücksendung des Kurzfragebogens reagierten, könnte im Vergleich zu den Teilnehmern der Hauptumfrage ein größeres Desinteresse an der Etablierung einer Ethikberatung unterstellt werden. Lediglich $20 \%$ der Teilnehmer gaben an, dass sie eine Ethikberatung nicht nutzen würden. Dieses Ergebnis ist überraschend niedrig und kann als positives Signal im Hinblick auf die Etablierung einer Ethikberatung gewertet werden. Knapp 40\% sahen sich zu oft mit Fragebögen konfrontiert, mehr als die Hälfte der Antwortenden des Kurzfragebogens gab an, im Moment keine Zeit zu haben. Dieses Ergebnis verwundert nicht, der Zeitmangel deutscher Ärzte ist bekannt, im Vergleich zu anderen europäischen Ländern haben deutsche Ärzte durchschnittlich $30 \%$ weniger Zeit für ihre Patienten (Sawicki und Bastian 2008) und damit vermutlich auch für Umfragen.

\subsection{Praktische Implikationen und weiterer Forschungsbedarf}

Die Frage, ob eine ambulante Ethikberatung etabliert werden sollte, kann diese Studie letztlich nicht beantworten. Ein generelles Interesse von Hausärzten an einer ambulanten Ethikberatung besteht in jedem Fall, das zeigt die hohe Antwortquote dieser Studie. Das Ergebnis dieser Arbeit kann dahingehend gedeutet werden, dass eine Ethikberatung nicht von allen Hausärzten als eine geeignete Unterstützung empfunden wird, aber doch von vielen. Ähnliches spiegeln die Ergebnisse zu Balint- und Supervisionsgruppen, sie scheinen sich in der Konfliktlösung bewährt zu haben, allerdings nimmt nur eine Minderheit der Ärzte diese Möglichkeit wahr.

Viele Ärzte wünschten sich eine Hilfestellung bei der Lösung von Konflikten (junge Ärzte eher als ältere Ärzte, Frauen eher als Männer). Häufig sind sie unsicher, was sie von solch einer Institution erwarten können und ob diese nicht selbst zu einer Belastung werden könnte. Möglicherweise sind die unklare Finanzierungsgrundlage einer Ethikberatung, ihr Zeitaufwand und die Vergütung von Hausärzten ein weiterer Unsicherheitsfaktor. 
Die Mehrheit der befragten Ärzte wünschte sich keinen unmittelbaren Kontakt mit Ethikberatern, sie bevorzugten eine telefonische Beratung und retrospektive Fallbesprechungen. Beratungen in der Praxis oder am Patientenbett wurden größtenteils abgelehnt. Als Ethikberater wurden vorwiegend ärztliche Kollegen mit Erfahrung in der ambulanten Patientenversorgung, medizinischem Fachwissen und Kenntnissen im Medizinrecht favorisiert; die Vergütung einer Beratung war für viele Ärzte nicht zwingend notwendig. Um auch die zweifelnden Ärzte zu überzeugen, sollten die Aufgabenbereiche einer ambulanten Ethikberatung und ihre Arbeitsweise bekannter gemacht werden. Mitglieder des ambulanten Ethikkomitees dürfen sich nicht aufdrängen; die Freiwilligkeit einer Beratung sollte im Vordergrund stehen, wobei individuell auf die Bedürfnisse der Ratsuchenden eingegangen werden sollte. Es gibt Fälle, in denen klinische Ethikberatungen vor allem aufgrund der positiven Berücksichtigung in Bezug auf Zertifizierungsverfahren etabliert wurden, die Nachteile dieses Verfahrens wurden erläutert. Inwieweit ein Zertifizierungsverfahren für ambulante Ethikberatungen sinnvoll ist, bleibt abzuwarten.

Nicht bestimmt wurden mögliche Unterschiede zwischen Landärzten und in Städten praktizierenden Ärzten. Städte und Dörfer unterscheiden sich in vielerlei Hinsicht. So können im Dorf lebende alte Menschen häufiger auf tragfähige und umfangreiche soziale Netze zurückgreifen, während städtisch wohnende Menschen tendenziell eher isoliert und anonym leben. Dörfer sind im Hinblick auf die Infrastruktur benachteiligt, zudem sind Alten- und Pflegeheime in Dörfern seltener zu finden (Garms-Homolová und Korte 1993). Um die ambulante Ethikberatung diesen örtlichen Besonderheiten anzupassen, sollten diese Unterschiede berücksichtigt werden. Weitere offene Fragen betreffen die Organisationsstruktur einer ambulanten Ethikberatung. Wer nimmt die Anfrage entgegen? Wer darf sie einberufen? Wo hat sie ihren festen Sitz? Wie wird sie dokumentiert? Wie wird sie finanziert? Die Beantwortung der genannten Fragen war nicht Ziel dieser Arbeit, liefert aber Anhaltspunkte für weiterführende Studien. 


\section{Zusammenfassung}

Hintergrund. Klinische Ethikberatungen haben sich in Deutschland (und vielen anderen Ländern) mittlerweile etabliert und bewährt. Geschulte Ethikberater helfen Ärzten im klinischen Alltag bei der Lösung von Konflikten. Beratungsmöglichkeiten im ambulanten Bereich gibt es dagegen kaum, obwohl Hausärzte natürlich auch mit belastenden ethischen Konflikten konfrontiert sind, beispielsweise in der Betreuung von Hospizpatienten.

Fragestellung. Wie häufig erleben Hausärzte ethische Konflikte und wie groß ist der Bedarf nach einer ambulanten Ethikberatung?

Methode. Im Bundesland Niedersachsen erhielten 1000 Hausärzte einen standardisierten Fragebogen zum Thema Konflikte in der ambulanten Versorgung und Wunsch nach einer Ethikberatung. Die Auswertung erfolgte vorwiegend deskriptiv; Faktoren für den Wunsch nach einer Ethikberatung wurden mittels multipler logistischer Regression bestimmt.

Ergebnisse. Die Antwortrate der Studie lag bei 45,6\%. Drei von zwölf Konflikten wurden von einem Großteil der Befragten ca. einmal im Monat erlebt: „Fortsetzung einer potentiell kurativen Therapie, die schlecht vertragen wird“, „Absetzen von Arzneien bei mittlerweile fraglichem Nutzen für den Patienten“, „Sinnhaftigkeit diagnostischer Maßnahmen zur Früherkennung bei bereits schwerkranken Patienten“. Zwei Drittel aller Befragten nahmen an Qualitätszirkeln teil, etwas mehr als die Hälfte hat dort Unterstützung erhalten. Ein knappes Drittel der befragten Hausärzte $(30,6 \%)$ wünschte sich eine ambulante Ethikberatung, mehr Frauen als Männer (Odds Ratio 1,72; 95\%-Konfidenzintervall: 1,1-2,6; p: 0,01); 37\% der Befragten wünschten keine Ethikberatung. Dennoch empfand über die Hälfte der Befragten die Etablierung einer Ethikberatung als sinnvoll, ebenfalls mehr Frauen als Männer (Odds Ratio 1,82; 95\%-Konfidenzintervall: $1,2-2,7 ; p: 0,003)$. Ethikberatungen sollten vor allem telefonisch $(81,5 \%)$ erreichbar sein und retrospektive Fallbesprechungen (55,6\%) anbieten. Beratungen sollten von Ärzten (55,7\%), vor allem von Hausärzten (60,1\%) geleistet werden. Neben medizinischem Fachwissen (89\%) und Erfahrungen in der ambulanten Patientenversorgung $(89,5 \%)$ sollten sich Ethikberater im Medizinrecht (86,5\%) auskennen. Eine Vergütung der Hausärzte für die Inanspruchnahme einer Ethikberatung spielte zumindest für $25 \%$ der Befragten eine Rolle.

Schlussfolgerung. Ethische Konflikte in Hausarztpraxen sind vergleichsweise häufig, Unterstützungsmöglichkeiten gibt es selten. Eine ambulante Ethikberatung trifft den Bedarf von einer Mehrheit der Befragten, insbesondere den von Hausärztinnen. Sie könnte dazu beitragen, Hausärzten bei der Bewältigung ethischer Konflikte zu helfen. 


\section{Literaturverzeichnis}

Abele AE (2003): The dynamics of masculine-agentic and feminine-communal traits: Findings from a prospective study. J Pers Soc Psychol $\underline{85}, 768-776$

Abholz H-H (2008): Komplexität bei der Behandlung in der Allgemeinmedizin - Schwierigkeiten der Bestimmung von Outcome-Parametern. Z Evid Fortbild Qual Gesundhwes $\underline{102}$, 351-356

Abholz H-H, Pillau H: Der Notfall in der Allgemeinmedizin. In: Kochen MM: Duale Reihe Allgemeinmedizin und Familienmedizin. 4. überarb. Auflage; Georg-Thieme-Verlag, Stuttgart 2012, 39-44

Ahrens D, Behrens G, Himmel W, Kochen MM, Chenot J-F (2012): Appropriateness of proton pump inhibitor recommendations at hospital discharge and continuation in primary care: PPI in primary care after hospital discharge. Int J Clin Pract $\underline{66}, 767-773$

Akademie für Ethik in der Medizin e. V. (2010): Standards für Ethikberatung in Einrichtungen des Gesundheitswesens: Vorstand der Akademie für Ethik in der Medizin e. V. Ethik Med $\underline{22}, 149-153$

Aleksandrova S (2008): Survey on the experience in ethical decision-making and attitude of Pleven University Hospital physicians towards ethics consultation. Med Health Care Philos 11, 35-42

Alexander S (1962): They decide who lives, who dies. Life $\underline{53}, 102-127$

Atteslander P: Methoden der empirischen Sozialforschung. 9., neu bearb. und erw. Auflage; De Gruyter, Berlin New York 2000

Aulisio MP, Arnold RM, Youngner SJ (2000): Health Care Ethics Consultation: Nature, Goals, and Competencies: A Position Paper from the Society for Health and Human Values-Society for Bioethics Consultation Task Force on Standards for Bioethics Consultation. Ann Intern Med 133, 59-69

Ayres PJ (1996): Rationing health care: Views from general practice. Soc Sci Med $\underline{42}, 1021-$ 1025 
Bauer AW (1993): Der Hippokratische Eid. Deutsche Übersetzung und medizinhistorischer Kommentar.

www.umm.uni-heidelberg.de/ag/gte/bauer_hippokratischer_eid.pdf (Zugriff: 05.06.2016)

Beecher HK, Adams RD, Barger AC, Curran WJ, Denny-Brown D, Farnsworth DL, Folch-Pi J, Mendelsohn EI, Merrill JP, Murray J (1968): A definition of irreversible coma. JAMA $\underline{205}, 337-340$

Beitzel KI, Ertl L, Grosse C, Reiser M, Ertl-Wagner B (2011): Berufszufriedenheit von Radiologen in Deutschland - aktueller Stand. Fortschr. Röntgenstr. 183, 749-757

Beleites E (1998): Wegweiser für ärztliches Handeln. Dtsch Arztebl $\underline{95}, 1851-1851$

Bockenheimer-Lucius G, May AT (2007): Ethikberatung-Ethik-Komitee in Einrichtungen der stationären Altenhilfe (EKA). Ethik Med 19, 331-339

Bortz J, Döring N: Forschungsmethoden und Evaluation für Human- und Sozialwissenschaftler: Limitierte Sonderausgabe. 4. überarb. Auflage; Springer-Verlag, Berlin Heidelberg 2015

Bradburn NM, Sudman S, Wansink B: Asking Questions: The Definitive Guide to Questionnaire Design - For Market Research, Political Polls, and Social and Health Questionnaires. Rev ed.; John Wiley \& Sons Inc, San Francisco 2004

Braunack-Mayer AJ (2001): What makes a problem an ethical problem? An empirical perspective on the nature of ethical problems in general practice. J Med Ethics 27, 98-103

Bundesagentur für Arbeit (2011): Der Arbeitsmarkt in Deutschland - Gesundheits- und Pflegeberufe.

http://statistik.arbeitsagentur.de/Statischer-

Content/Arbeitsmarktberichte/Fachkraeftebedarf-Stellen/Fachkraefte/BA-FKEngpassanalyse-2014-12.pdf (Zugriff: 06.06.2016)

Bundesärztekammer (2008): Beschlussprotokoll des 111. Deutschen Ärztetages vom 20. bis 23. Mai 2008 in UIm. http://www.bundesaerztekammer.de/aerztetag/beschlussprotokolle-ab-1996/111-daet2008/ (Zugriff: 06.06.2016) 
Bundesärztekammer (2011): Grundsätze der Bundesärztekammer zur ärztlichen Sterbebegleitung. Dtsch Ärztebl $\underline{3}, 138-140$

Burger M (2008): Die Balintgruppe als professioneller Stabilisierungsfaktor für Beraterlnnen und Führungskräfte. Gruppendynamik und Organisationsberatung $\underline{39}$, 316-325

Connelly JE, DalleMura S (1988): Ethical problems in the medical office. JAMA $\underline{260}, 812-$ 815

Coors DM, Simon A, Stiemerling M: Ethikberatung in Pflege und ambulanter Versorgung: Modelle und theoretische Grundlagen. 1. Auflage; Jacobs Verlag, Lage 2015

Craig JM, May T (2006): Evaluating the outcomes of ethics consultation. J Clin Ethics 17, 168-180

Deutsche Gesellschaft für Palliativmedizin (2007): Spirituelle Begleitung in der Palliativversorgung. Konzept des Arbeitskreises Spirituelle Begleitung der Deutschen Gesellschaft für Palliativmedizin.

https://www.dgpalliativmedizin.de/images/stories/pdf/fachkompetenz/070709\%20Spiritue Ile\%20Begl\%20in\%20Pm\%20070510.pdf (Zugriff am 05.06.2016)

Deutscher Evangelischer Krankenhausverband e.V. \& Katholischer Krankenhausverband Deutschlands e.V. (1997), Ethik-Komitee im Krankenhaus.

http://www.ev-medizinethik.de/meta_downloads/9856/dekv_-_ethik-komitee.pdf (Zugriff: 06.06.2016)

Donner-Banzhoff N (2008): Umgang mit Unsicherheit in der Allgemeinmedizin. Z Evid Fortbild Qual Gesundhwes 102, 13-18

Dörries A, Hespe-Jungesblut K (2007): Die Implementierung Klinischer Ethikberatung in Deutschland: Ergebnisse einer bundesweiten Umfrage bei Krankenhäusern. Ethik Med $\underline{19}, 148-156$

DuVal G, Sartorius L, Clarridge B, Gensler G, Danis M (2001): What triggers requests for ethics consultations? West $\mathrm{J}$ Med $\underline{175}, 24-30$

DuVal G, Clarridge B, Gensler G, Danis M (2004): A national survey of US internists' experiences with ethical dilemmas and ethics consultation. J Gen Intern Med 19, 251-258 
Fox E, Myers S, Pearlman RA (2007): Ethics Consultation in United States Hospitals: A National Survey. Am J Bioeth $\underline{7}, 13-25$

Frankfurter Allgemeine Zeitung (2005): Umgang mit dem Tod: Terri Schiavo: „Mord“ oder „Erlösung“? Frankfurter Allgemeine Zeitung, Frankfurt, 01.04.2005 http://www.faz.net/aktuell/gesellschaft/menschen/umgang-mit-dem-tod-terri-schiavomord-oder-erloesung-1215418.html (Zugriff: 05.06.2016)

Frese M (1987): Arbeit und psychische Störungen. Gewerkschaftliche Monatshefte 11, 679691

Frewer A, Säfken C (2003): Ärztliche Schweigepflicht und die Gefährdung Dritter. Ethik Med $\underline{15}, 15-24$

Gágyor I: Ethikberatung für Hausärzte bei Patienten am Lebensende; In: Bruns F, Frewer A, May AT (Hrsg.): Ethikberatung in der Medizin. Springer-Verlag, Berlin Heidelberg 2012, 141-149

Gágyor I, Lüthke A, Jansky M, Chenot J-F (2013): End of life care in general practice: results of an observational survey with general practitioners. Schmerz $\underline{27}, 289-295$

Gágyor I, Himmel W, Pierau A, Chenot J-F (2016): Dying at home or in the hospital? An observational study in German general practice. Eur J Gen Pract 22, 9-15

Garfinkel D, Mangin D (2010): Feasibility study of a systematic approach for discontinuation of multiple medications in older adults: addressing polypharmacy. Arch Intern Med $\underline{170}$, 1648-1654

Garms-Homolová V, Korte W: Altern in der Stadt und auf dem Lande - Unterschiede oder Angleichung? In: Naegele G, Tews HP (Hrsg.); Lebenslagen im Strukturwandel des Alters, VS Verlag für Sozialwissenschaften, Wiesbaden 1993, 215-233

Gerdes B, Richter G (1999): Ethik-Konsultationsdienst nach dem Konzept von JC Fletcher an der University of Virginia, Charlottesville, USA. Ethik Med 11, 249-261

Gologan R, Aziriu S, Obertacke U, Schreiner U (2014): Medizinische und soziodemographische Aspekte häuslicher Gewalt: Systematische Auswertung von Patientendaten einer Notaufnahme eines Krankenhauses der Maximalversorgung. Unfallchirurg 117, 528-532 
Handel E (2003): Das Beziehungsgeflecht Patientin/Patient, Angehörige und Betreuungsteam. Eine systematische Betrachtungsweise.

www.zfg.uzh.ch/static/2003/handel_beziehungsgeflecht.pdf (Zugriff: 05.06.2016)

Harringer W, Hoby G, Qualitätszirkel ZOC, Rorschach R (2009): Patientenverfügung in der hausärztlichen Praxis. PrimaryCare $\underline{9}, 56-58$

Harth W, Wendler M, Linse R (2003): Lifestyle-Medikamente und körperdysmorphe Störungen. Dtsch Ärztebl 100, A128-A131

Heßling A, Marx G, Simon A, Nauck F, Hummers-Pradier E, Gágyor I (2014): Ethikberatung für die ambulante Versorgung - eine qualitative Pilotstudie. ZS Palliativmed $\underline{15}, 124$

Hibbeler B (2011): Ärzte und Pflegekräfte: Ein chronischer Konflikt. Dtsch Arztebl 108, A2138 $-\mathrm{A} 2145$

Hiemetzberger M: Ethik in der Pflege. 2. Auflage; Facultas.Universitätsverlag, Wien 2010

Himmel W, Kochen MM, Sorns U, Hummers-Pradier E (2004): Drug changes at the interface between primary and secondary care. Int J Clin Pharmacol Ther $\underline{42}, 103-109$

Höger C, Reiter-Theil S, Reiter L, Derichs G, Kastner-Voigt M, Schulz T (1997): Fallbezogene ethische Reflexion. System Familie 10, 174-179

Hummers-Pradier E, Scheidt-Nave C, Martin H, Heinemann S, Kochen MM, Himmel W (2008): Simply no time? Barriers to GPs' participation in primary health care research. Fam Pract 25, 105-112

Hurst SA, Slowther A-M, Forde R, Pegoraro R, Reiter-Theil S, Perrier A, Garrett-Mayer E, Danis M (2006): Prevalence and Determinants of Physician Bedside Rationing: Data from Europe. J Gen Intern Med 21, 1138-1143

Hurst SA, Reiter-Theil S, Slowther A-M, Pegoraro R, Forde R, Danis M (2008): Should ethics consultants help clinicians face scarcity in their practice? J Med Ethics $\underline{34}, 241-246$

Jacob R, Eirmbter WH: Allgemeine Bevölkerungsumfragen: Einführung in die Methoden der Umfrageforschung mit Hilfen zur Erstellung von Fragebögen. (Lehr- und Handbücher der Soziologie), Reprint 2014; De Gruyter Oldenbourg, Berlin Boston 2000 
Janus K, Amelung V, Baker L, Gaitanides M, Rundall T, Schwartz F (2009): Sind amerikanische Ärzte zufriedener? - Ergebnisse einer internationalen Studie unter Ärzten an Universitätskliniken. Gesundheitswesen $\underline{71}, 210-217$

Jonsen AR (2007): The God Squad and the Origins of Transplantation Ethics and Policy. J Law Med Ethics 35, 238-240

Jox R, Winkler E, Borasio G (2012): Änderung des Therapieziels am Lebensende: Effekte einer Klinik-Leitlinie. DMW - Dtsch Med Wochenschr 137, 829-833

Kalager G, Førde R, Pedersen R (2011): Is the discussion of patient cases in clinical ethicscommittees useful? Tidsskr Nor Laegeforen 131, 118-121

Karazivan P, Pless C (2011): Should family physicians treat members of the same family? Can Fam Physician $\underline{57}$, 402-404

Kassenärztliche Bundesvereinigung (2010): Richtlinien der Kassenärztlichen Bundesvereinigung für Verfahren zur Qualitätssicherung.

http://www.bvf.de/pdf/richtlinien/KBV_QS_Richtlinien.pdf (Zugriff: 06.06.2016)

Kassenärztliche Bundesvereinigung (2015): Statistische Kerndaten aus dem Bundesarztregister zum Stichtag 31.12.2014.

http://www.kbv.de/media/sp/2014_12_31_Statistische_Kerndaten_BAR.pdf (Zugriff: 05.06.2016)

Kemper A, Kölch M, Fangerau H, Fegert JM (2010): Ärztliche Schweigepflicht bei Kindeswohlgefährdung: Mehr Handlungssicherheit durch die neuen Kinderschutzgesetze? Ethik Med 22, 33-47

Kettner M, May A (2004): Klinische Ethik-Komitees. Ihre Organisationsformen und ihr moralischer Anspruch in Theorie und Praxis; Ein Forschungsprojekt am Wissenschaftszentrum Nordrhein-Westfalen.

http://web.ev-akademie-tutzing.de/cms/get_it.php?ID=70 (Zugriff: 05.01.2017)

Klein S, Porst R: Mail Surveys. Ein Literaturbericht. ZUMA-Technischer Bericht 10/2000, http://www.gesis.org/fileadmin/upload/forschung/publikationen/gesis_reihen/gesis_metho denberichte/2000/00_10.pdf. (Zugriff: 06.06.2016) 
Kravitz RL, Epstein RM, Feldman MD, Franz CE, Azari R, Wilkes MS, Hinton L, Franks P (2005): Influence of patients' requests for direct-to-consumer advertised antidepressants: a randomized controlled trial. Jama $\underline{293}, 1995-2002$

Kreft M, Kliesch S, Kotterba S: Ambulantes Ethikkomitee Ammerland/Uplengen: Ausgangslage und auslösende Faktoren. In: Coors DM, Simon A, Stiemerling M (Hrsg.): Ethikberatung in Pflege und ambulanter Versorgung: Modelle und theoretische Grundlagen. 1. Auflage; Jacobs-Verlag, Lage 2015, 151-161

Kruip G, Winkler K: Ethisches Lernen in der allgemeinen Erwachsenenbildung. Katholische Bundesarbeitsgemeinschaft für Erwachsenenbildung, Mainz 2007

Kutner J, Blatchford P, Taylor D, Ritchie C, Bull J, Fairclough D, Hanson L, LeBlanc T, Samsa G, Wolf $S$ (2015): Safety and benefit of discontinuing statin therapy in the setting of advanced, life-limiting illness: A randomized clinical trial. JAMA Intern Med $\underline{175}, 691-700$

Leiblein A, Oglesby S (1993): Akzeptanz von Umfragen: vier Länder im Vergleich. Eigenstudie der LINK-Inst. für Markt- und Sozialforschung, http://www.link.ch/wp-content/uploads/2013/08/LINK_Focus_01.pdf (Zugriff: 06.06.2016)

Magon H (2014): Überlegungen in zwei Teilen - Teil 2 - Ängste und Bedürfnisse schwerkranker und sterbender Menschen. ZS Palliativmed 15, 154-157

Maier T (2001): Die Praxis der Fürsorgerischen Freiheitsentziehung. Praxis $\underline{90}$, 1575-1581

Maio G (2002): Braucht die Medizin klinische Ethikberater? Dtsch Med Wochenschr 127, 2285-2288

Marti C, Bovet U (2004): Patientenverfügungen - eine Herausforderung für Patient und Arzt. Schweiz Arzteztg $\underline{85}, 560-561$

May AT (2011): Ethikberatung in Einrichtungen des Gesundheitswesens. Forum $\underline{26}$, 14-16

May AT, Beule G, Gollan K, Heinemann W, Oestermann B: Curriculum zur Qualifikation für Mitglieder von Ethik-Komitees in kirchlichen Einrichtungen des Gesundheitswesens. In: Heinemann W, Maio G (Hrsg.): Ethik in Strukturen bringen: Denkanstöße zur Ethikberatung im Gesundheitswesen. 1. Auflage; Herder, Freiburg 2010, 247-264

Menold N: Schriftlich-postalische Befragung. SDM Survey Guidelines, Mannheim 2014 
Möller JC, Stiasny K, Cassel W, Peter JH, Krüger HP, Oertel WH (2000): "Schlafattacken" bei Parkinson-Patienten Eine Nebenwirkung von Nonergolin-Dopaminagonisten oder ein Klasseneffekt von Dopamimetika? Nervenarzt $\underline{71}$, 670-676

Morison RS (1981): Bioethics after Two Decades. Hastings Cent Rep 11, 8-12

Müller P (2004): Zwangseinweisungen nehmen zu. Dtsch Arztebl 101, 2794-2798

Müller S (2010): Revival der Hirntod-Debatte: Funktionelle Bildgebung für die HirntodDiagnostik. Ethik Med 22, 5-17

Nauck F (2011): Ethische Aspekte in der Therapie am Lebensende. Med Klin 106, 137-148

Neitzke G: Praxis der Klinischen Ethikberatung. In: Dörries A, Neitzke G, Simon A, Vollmann $J$ (Hrsg.): Klinische Ethikberatung: Ein Praxisbuch. Kohlhammer, Stuttgart 2008, 58-86

Neitzke G: Formen und Strukturen Klinischer Ethikberatung. In: Vollmann J, Schildmann J, Simon A (Hrsg.): Klinische Ethik: aktuelle Entwicklungen in Theorie und Praxis. Campus Verlag, Frankfurt/Main 2009, 37-56

Netzwerk Hospiz (2012) - Flyer Außerklinische Ethikberatung - Kliniken Südostbayern AG. http://www.kliniken-suedostbayern.de/files/PDF-

Dokumente/ausserklinische_Ethik_Netzwerk_Hospiz_web.pdf (Zugriff: 06.06.2016)

Parzeller M, Wenk M, Rothschild MA (2005): Die ärztliche Schweigepflicht. Dtsch Arztebl 102, A289-A297

Porst R (1998): Im Vorfeld der Befragung: Planung, Fragebogenentwicklung, Pretesting. ZUMA Arbeitsbericht. http://www.s-hb.de/ klaus.boenkost/pdf-docs/Im-Vorfeld-derBefragung.pdf (Zugriff: 06.06.2016)

Porst R: Frageformulierung. In: Baur N, Blasius J (Hrsg.): Handbuch Methoden der empirischen Sozialforschung. Springer-Verlag, Wiesbaden 2014, 687-699

Prüfer P, Rexroth M (2005): Kognitive Interviews. ZUMA.

http://www.gesis.org/fileadmin/upload/forschung/publikationen/gesis_reihen/howto/How_ to15PP_MR.pdf (Zugriff: 05.06.2016) 
Prüter C (2008): Rechtliche Grundlagen der Behandlung psychiatrischer Patienten im Notarztdienst. Notfall Rettungsmed 11, 552-556

Rabe JC (2016): Wahlen: Das Prinzip des Pi-mal-Daumen. Süddeutsche Zeitung, München, 20. März.2016

http://www.sueddeutsche.de/kultur/wahlen-das-prinzip-des-pi-mal-daumen-1.2915366 (Zugriff: 05.06.2016)

Rappe-Giesecke K: Gruppensupervision und Balintgruppenarbeit. In: Pühl H (Hrsg.): Handbuch der Supervision 2: Grundlagen, Praxis, Perspektiven. Spiess-Verlag, Berlin 1994, 72-84

Rappe-Giesecke K (2000): Balint-Gruppenarbeit aus kommunikationswissenschaftlicher Sicht. Balint J 1 , 36-42

Reiter-Theil S (1999): Ethik in der Klinik-Theorie für die Praxis: Ziele, Aufgaben und Möglichkeiten des Ethik-Konsils. Ethik Med 11, 222-232

Reiter-Theil S (2000): Ethics consultation on demand: concepts, practical experiences and a case study. J Med Ethics 느, 198-203

Roebel A, Wenk M, Parzeller M (2009): Postmortale ärztliche Schweigepflicht. Rechtsmedizin $\underline{19}, 37-52$

Sampson R, Barbour R, Wilson P (2016): The relationship between GPs and hospital consultants and the implications for patient care: a qualitative study. BMC Fam Pract; 17: in press

Sawicki PT, Bastian H (2008): German health care: a bit of Bismarck plus more science. BMJ $\underline{337}, 1142-1145$

Schneider W (2013): Medikalisierung sozialer Prozesse. Psychotherapeut 토, 219-236

Schnell R, Hill PB, Esser E: Methoden der empirischen Sozialforschung. 6. völlig überarbeitete und erweiterte Auflage; Oldenbourg Wissenschaftsverlag, München 1998

Schochow M, May A, Schnell D, Steger F (2014): Wird Klinische Ethikberatung in Krankenhäusern in Deutschland implementiert? DMW - Dtsch Med Wochenschr 139, 21782183 
Shih T-H, Xitao Fan (2008): Comparing Response Rates from Web and Mail Surveys: A Meta-Analysis. Field Methods $\underline{20}, 249-271$

Simon A (2001): Ethics Committees in Germany: An Empirical Survey of Christian Hospitals. HEC Forum $\underline{13}$, 225-231

Simon A: Ethikberatung in Pflege und ambulanter Versorgung: Eine Landkarte der unterschiedlichen Modelle. In: Coors DM, Simon A, Stiemerling M (Hrsg.): Ethikberatung in Pflege und ambulanter Versorgung: Modelle und theoretische Grundlagen. 1. Auflage; Jacobs Verlag, Lage 2015, 68-77

Simon A, May AT, Neitzke G (2005): Curriculum „Ethikberatung im Krankenhaus“. Ethik Med 17, 322-326

Slowther A (2009): Ethics Case Consultation in Primary Care: Contextual Challenges for Clinical Ethicists. Camb Q Healthc Ethics $\underline{18}, 397-405$

Steinert T, Hinüber W, Arenz D, Röttgers HR, Biller N, Gebhardt RP (2001): Ethische Konflikte bei der Zwangsbehandlung schizophrener Patienten Entscheidungsverhalten und Einflussfaktoren an drei prototypischen Fallbeispielen. Nervenarzt $\underline{72}, 700-708$

Steinkamp N, Gordijn B (2001): The Two-Layer Model of Clinical Ethics and a Training Program for the Malteser Hospital Association. HEC Forum 13, 242-254

Stokes ME, Davis CS, Koch GG: Categorical Data Analysis Using SAS, 3. Auflage; SAS Institute, New York 2012

Strätling MW, Sedemund-Adib B (2013): Ethische Kernkompetenzen in die Medizin zurückholen. Dtsch Arztebl 110, A825-A828

Synofzik M, Maetzler W (2007): Wie sollen wir Patienten mit Demenz behandeln? Die ethisch problematische Funktion der Antidementiva. Ethik Med 19, 270-280

Tapper EB (2013): Consults for conflict: the history of ethics consultation. Proc Bayl Univ Med Cent 26, 417-422

Thürmann PA (2013): Weniger wäre tatsächlich mehr - die Arzneimittelversorgung alter Menschen. Z Evid Fortbild Qual Gesundhwes 107, 148-152 
Ulsenheimer K (2009): Hirntod und Intensivmedizin: Juristische Probleme. Anaesthesist $\underline{58}$, 722-727

Ulsenheimer K: Arztstrafrecht in der Praxis. C.F. Müller, Heidelberg 2014

Vollmann J: Klinische Ethikkomitees und Ethikberatung in Deutschland: Bisherige Entwicklung und zukünftige Perspektiven. Bioethica Forum 1 2008a, 33-39

Vollmann J: Ethikberatung an deutschen Universitätskliniken. Empirische Ergebnisse und aktuelle Entwicklungen. In: Gross D, May AT, Simon A (Hrsg.): Beiträge zur Klinischen Ethikberatung an Universitätskliniken. 1. Auflage; LIT, Berlin Münster 2008b, 31-48

Vollmann J: Ethische Falldiskussion. In: Dörries A, Neitzke G, Simon A, Vollmann J (Hrsg.): Klinische Ethikberatung: Ein Praxisbuch. Kohlhammer, Stuttgart 2008c, 87-101

Von Eisenhart Rothe A (2015): Aus der Praxis: Gerechtes Gehalt, das geht. ergopraxis $\underline{8}$, 44-45

Wallenstein J, Ziegler B, Kreid E: Fallstudie zum Change Management in der Arzneimittelindustrie: Pharmaaußendienst im Umbruch. In: Busse R, Schreyögg J, Gericke C (Hrsg.): Management im Gesundheitswesen. Springer-Verlag, Berlin 2006, 447-452

Wandrowski J, Schuster T, Strube W, Steger F (2012): Medizinethische Kenntnisse und moralische Positionen von Ärztinnen und Ärzten aus Bayern. Dtsch Arztebl 109, 141-147

Wasson K, Anderson E, Hagstrom E, McCarthy M, Parsi K, Kuczewski M (2016): What Ethical Issues Really Arise in Practice at an Academic Medical Center? A Quantitative and Qualitative Analysis of Clinical Ethics Consultations from 2008 to 2013. HEC Forum $\underline{28}$, 217-228

Wermeling M, Himmel W, Behrens G, Ahrens D (2014): Why do GPs continue inappropriate hospital prescriptions of proton pump inhibitors? A qualitative study. Eur J Gen Pract $\underline{20}$, 174-180

Winkler EC, Borasio GD, Jacobs P, Weber J, Jox RJ (2012): Münchner Leitlinie zu Entscheidungen am Lebensende. Ethik Med 24 , 221-234

Yen BM, Schneiderman LJ (1999): Impact of pediatric ethics consultations on patients, families, social workers, and physicians. J Perinatol $\underline{19}$, 373-378 
Zentrale Ethikkommission (2006): Bekanntmachungen: Stellungnahme der Zentralen Kommission zur Wahrung ethischer Grundsätze in der Medizin und ihren Grenzgebieten (Zentrale Ethikkommission) bei der Bundesärztekammer zur Ethikberatung in der klinischen Medizin (24. Januar 2006). Dtsch Arztebl 103, A1703-A1707

Ziegler A: Ärztliche Schweigepflicht. In: Hick C (Hrsg.): Klinische Ethik. Springer-Verlag, Ber$\operatorname{lin} 2007,41-53$

\section{Internetquellen:}

Allgemeinmedizin Göttingen 2015

http://www.allgemeinmedizin.med.uni-goettingen.de/de/content/forschung/400.html;

Zugriff: 19.02.2016

Christliche Krankenhäuser in Deutschland 2016

http://www.christliche-krankenhaeuser.de/\#/wer-wir-sind;

Zugriff: 17.02.2016

Deutsche Gesundheitsnachrichten 2012

http://www.deutsche-gesundheits-nachrichten.de/2012/04/30/unhaltbar-jeden-montag-sitzen-

8-prozent-der-deutschen-in-einem-wartezimmer/;

Zugriff: 16.02.2016 


\section{Anhang}

\subsection{Anschreiben Hauptfragebogen}

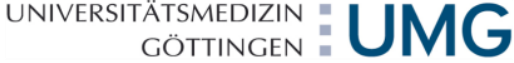

Sehr geehrte(r) Frau/Herr X,

liebe(r) Frau/Herr Kollegin/Kollege,

im Krankenhaus gibt es die Möglichkeit einer klinischen Ethikberatung. Dabei beraten Mitglieder eines Ethikkomitees Ärzte bei konflikthaften Situationen. Für den ambulanten Bereich gibt es diese Möglichkeit der Unterstützung bisher nicht.

Nun möchten wir eine für Niedersachsen repräsentative Gruppe von Hausärztinnen und Haus ärzten zur Häufigkeit und Belastung durch ethische Konflikte befragen, um festzustellen, ob es häufig zu Konflikten kommt und es einen Bedarf an ambulanter Ethikberatung gibt. Der folgende Fragebogen ist das Ergebnis aus Gesprächen mit Hausärzten. Einige berichteten dort von ethischen Konflikten, die sie in ihrer hausärztlichen Tätigkeit erleben und welche Formen der Unterstützung sie sich dabei vorstellen könnten. Die im Fragebogen genannten ethischen Konflikte waren für Ihre Kolleginnen und Kollegen ,typische“ Beispiele, z. T. häufig, z. T. selten erlebt. Wie sehen Sie das?

Wir hoffen auf Ihre Mitarbeit und bitten darum, den Fragebogen auch auszufüllen, wenn Sie nicht an der Errichtung einer ambulanten Ethikberatung interessiert sind. Bitte schicken Sie den Fragebogen in dem beigelegten Briefumschlag an uns zurück; das Deckblatt wird sofort nach Erhalt in unserem Institut zur Wahrung Ihrer Anonymität vernichtet.

Besten Dank für Ihre Teilnahme. Gerne stehen wir Ihnen für Rückfragen persönlich, telefonisch oder per E-Mail zur Verfügung.

Mit kollegialen Grüßen

Ihre

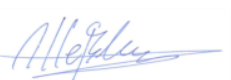

Dr. med. Arndt Heßling

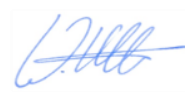

Prof. Dr. Wolfgang Himmel

Anlage Fragebogen „Ambulante Ethikberatung bei Konflikten in der hausärztlichen Versorgung 


\subsection{Deckblatt}

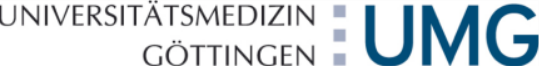

Universitätsmedizin Göttingen, 37099 Göttingen

Institut für Allgemeinmedizin

Prof. Dr. Eva Hummers-Pradier, Humboldtallee 38

Direktorin

37099 Göttingen Briefpost

Humboldtallee 38, 37073 Göttingen Adresse

0551 / 39-22638 Telefon

0551 / 39-9530 Fax
arndt.hessling@med.uni-goettingen.de E-Mail

Datum

\section{Fragebogen}

\section{Ambulante Ethikberatung bei Konflikten in der hausärztlichen Versorgung}

Bitte beachten Sie:

Dieses Deckblatt wird aus organisatorischen Gründen mit versendet, dadurch vermeiden wir ein zweites Anschreiben. Bitte trennen Sie es nicht vom Fragebogen ab. Dieses Blatt wird direkt nach Eingang in unserem Institut vernichtet. Ihre Angaben können zu keinem späteren Zeitpunkt Ihrer Person zugeordnet werden und bleiben anonym.

Besten Dank für Ihre Teilnahme!

Mit kollegialen Grüßen

Ihre

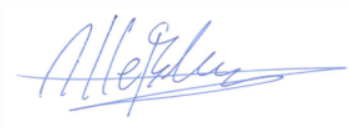

Dr. med. Arndt Heßling

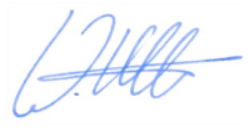

Prof. Dr. Wolfgang Himmel 


\subsection{Hauptfragebogen}

1. Wie oft sind Sie in Ihrer hausärztlichen Tätigkeit mit folgenden Entscheidungskonflikten konfrontiert?

\begin{tabular}{|c|c|c|c|c|c|}
\hline & $\begin{array}{l}\text { ca. } 1-\mathrm{mal} \\
\text { im } \\
\text { Monat }\end{array}$ & $\begin{array}{l}\text { ca. } 1-\text { mal } \\
\text { alle } \\
3 \text { Monate }\end{array}$ & $\begin{array}{l}\text { ca. } 1-\text { mal } \\
\text { alle } \\
6 \text { Monate }\end{array}$ & $\begin{array}{l}\text { seltener als } \\
\text { alle } \\
6 \text { Monate }\end{array}$ & $\begin{array}{l}\text { bisher } \\
\text { nicht } \\
\text { erlebt }\end{array}$ \\
\hline $\begin{array}{l}\text { Fortsetzung einer potentiell kurativen } \\
\text { Therapie, die schlecht vertragen wird. }\end{array}$ & $\square_{5}$ & $\square_{4}$ & $\square_{3}$ & $\square_{2}$ & $\square_{1}$ \\
\hline $\begin{array}{l}\text { Fortsetzung einer potentiell } \\
\text { lebensverlängernden Therapie bei infauster } \\
\text { Prognose, die schlecht vertragen wird. }\end{array}$ & $\square_{5}$ & $\square_{4}$ & $\square_{3}$ & $\square_{2}$ & $\square_{1}$ \\
\hline $\begin{array}{l}\text { Fortsetzung einer Therapie, wenn der } \\
\text { Patientenwille wegen einer progredienten } \\
\text { Erkrankung nicht mehr feststellbar ist. }\end{array}$ & $\square_{5}$ & $\square_{4}$ & $\square_{3}$ & $\square_{2}$ & $\square_{1}$ \\
\hline $\begin{array}{l}\text { Informationsweitergabe an Angehörige, } \\
\text { wenn nicht bekannt ist, ob der Patient dies } \\
\text { gestattet hat. }\end{array}$ & $\square_{5}$ & $\square_{4}$ & $\square_{3}$ & $\square_{2}$ & $\square_{1}$ \\
\hline $\begin{array}{l}\text { Nachträgliches Umsetzen einer } \\
\text { Patientenverfügung, die in einer } \\
\text { Notfallsituation nicht berücksichtigt wurde. }\end{array}$ & $\square_{5}$ & $\square_{4}$ & $\square_{3}$ & $\square_{2}$ & $\square_{1}$ \\
\hline $\begin{array}{l}\text { Einweisung von pflegebedürftigen } o . \\
\text { wesensveränderten Patienten in } \\
\text { Pflegeeinrichtungen gegen deren Willen. }\end{array}$ & $\square_{5}$ & $\square_{4}$ & $\square_{3}$ & $\square_{2}$ & $\square_{1}$ \\
\hline Selbstgefährdendes Patientenverhalten. & $\square_{5}$ & $\square_{4}$ & $\square_{3}$ & $\square_{2}$ & $\square_{1}$ \\
\hline $\begin{array}{l}\text { Vorgehen bei strafrechtlich relevanten } \\
\text { Informationen über Dritte (beispielsweise } \\
\text { häusliche Gewalt durch den Partner). }\end{array}$ & $\square_{5}$ & $\square_{4}$ & $\square_{3}$ & $\square_{2}$ & $\square_{1}$ \\
\hline $\begin{array}{l}\text { Konträre Behandlungsansichten zwischen } \\
\text { mir und einer stationären Einrichtung. }\end{array}$ & $\square_{5}$ & $\square_{4}$ & $\square_{3}$ & $\square_{2}$ & $\square_{1}$ \\
\hline $\begin{array}{l}\text { Absetzen von Arzneien bei mittlerweile } \\
\text { fraglichem Nutzen für den Patienten. }\end{array}$ & $\square_{5}$ & $\square_{4}$ & $\square_{3}$ & $\square_{2}$ & $\square_{1}$ \\
\hline $\begin{array}{l}\text { Sinnhaftigkeit diagnostischer Maßnahmen } \\
\text { zur Früherkennung bei bereits } \\
\text { schwerkranken Patienten. }\end{array}$ & $\square_{5}$ & $\square_{4}$ & $\square_{3}$ & 口 & $\square_{1}$ \\
\hline $\begin{array}{l}\text { Patienten und Angehörigen sind uneins } \\
\text { bzgl. diagnostischer bzw. therapeutischer } \\
\text { Maßnahmen. }\end{array}$ & $\square_{5}$ & $\square_{4}$ & $\square_{3}$ & $\square_{2}$ & 口 \\
\hline
\end{tabular}


2. Gibt es weitere Situationen, die aus Ihrer Sicht konflikthaft sind?

Nehmen Sie an Qualitätszirkeln teil?

口 ja

$\square_{0}$ nein [falls nein, weiter mit Frage 5]

4. Haben Sie in Qualitätszirkeln Unterstützung in konflikthaften Situationen erhalten?
$\square_{1}$ ja
$\square_{2}$ war bisher nicht nötig
$\square_{0}$ nein

5. Nehmen Sie an Supervisions- oder Balintgruppen teil?
$\square_{1 j \mathrm{a}}$
$\square_{0}$ nein [falls nein, weiter mit Frage 7]

6. Haben Sie in Supervisions- oder Balintgruppen Unterstützung in konflikthaften Situationen erhalten?
$\square_{1 j a}$
口 war bisher nicht nötig
$\square_{\text {nein }}$

7. Haben Sie sich in einer der oben genannten Situationen oder bei vergleichbaren Konflikten eine ambulante Ethikberatung gewünscht?
$\square_{1} \mathrm{ja}$
$\square_{2}$ bin mir nicht sicher
$\square_{0}$ nein

8. Halten Sie die Etablierung einer ambulanten Ethikberatung für sinnvoll?
$\square_{i}$ ja
- bin mir nicht sicher
$\square_{0}$ nein [falls nein, weiter mit Frage 13]

9. Welche Angebote sollte eine ambulante Ethikberatung umfassen?

(Mehrfachauswabl möglich)

a telefonische Beratung

- Beratung in der Praxis

$\square_{3}$ retrospektive Fallbesprechung

口 Beratung am Krankenbett

$\square_{5}$ Fortbildungen zu medizinethischen Fragestellungen für Hausärzte

$\square_{6}$ Leitlinienerstellung für widerkehrende medizinische Konfliktsituation

- Sonstige und zwar 
10. Welche beruflichen Hintergründe sollten ambulante Ethikberater haben?

(Mebrfachauswabl möglich)

$\square_{1}$ Fachärztin/ Facharzt für Allgemeinmedizin

$\square_{2}$ Ärztinnen / Ärzte im Allgemeinen

$\square_{3}$ Seelsorgerin /Seelsorger

$\square_{4}$ ambulante Pflegekräfte

$\square_{5}$ Sonstige und zwar:

11. Welche Qualifikationen und Eigenschaften sollten Ethikberater neben ihrer Schulung zum Berater haben?

(Mehrfachauswabl möglich)

$\square_{1}$ medizinisches Fachwissen

$\square_{2}$ Erfahrungen in der ambulanten Patientenversorgung

口 ${ }_{3}$ Kenntnisse bezüglich des Medizinrechts

a andere Qualifikationen und zwar:

12. Würden Sie eine ambulante Ethikberatung eher in Anspruch nehmen, wenn die aufgewendete Zeit vergütet würde?
$\square_{1 j \mathrm{a}}$
$\square_{2}$ bin mir nicht sicher
$\square_{\text {nein }}$

13. Sie sind:
a weiblich
$\square_{2}$ männlich

14. Wie alt sind Sie?

15. Haben Sie Zusatzqualifikationen?

口 ja, und zwar:

16. Seit wann arbeiten Sie in der ambulanten Patientenversorgung?

$\square_{1}$ Seit

17. Sie arbeiten in einer
口 Einzelpraxis
- Gemeinschaftspraxis
口 Praxisgemeinschaft 


\subsection{Anschreiben der zweiten Aussendung}

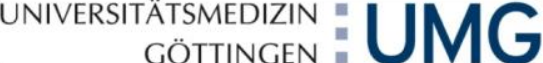

Universitätsmedizin Göttingen, 37099 Göttingen

Direktorin des Instituts für Allgemeinmedizin
Prof. Dr. Eva Hummers-Pradier, Humboldtallee 38

Institut für Allgemeinmedizin

Prof. Dr. Wolfgang Himme

37099 Göttingen Briefpost

Humboldtallee 38, 37073 Göttingen Adresse

0551 / 39-22638 Telefon

$0551 / 39-9530 \mathrm{Fax}$

arndt.hessling@med.uni-goettingen.de E-Mail

Datum

Ambulante Ethikberatung bei Konflikten in der hausärztlichen Versorgung

Sehr geehrte Frau/Herr X,

liebe(r) Frau/Herr Kollegin/Kollege,

im Dezember schickten wir Ihnen einen Fragebogen zum Thema „Ethikberatung in der hausärztlichen Versorgung“" und haben bisher keine Antwort erhalten. Wir haben Verständnis für Ihre starke Arbeitsbelastung, möchten Sie aber doch noch einmal bitten, sich im Interesse einer Verbesserung der hausärztlichen Betreuung an der Befragung zu beteiligen - unabhängig davon, ob Sie einer Ethikberatung eher positiv oder skeptisch gegenüberstehen.

Sollten Sie derzeit nicht in der Lage sein, den Fragebogen zu beantworten, haben wir ein Blatt beigelegt, auf dem Sie uns in aller Kürze - mit „einem Kreuz“ - mitteilen können, warum Sie nicht teilnehmen.

Besten Dank für Ihre Unterstützung. Gerne stehen wir Ihnen für Rückfragen persönlich, telefonisch oder per E-Mail zur Verfügung.

Mit kollegialen Grüßen

Ihre

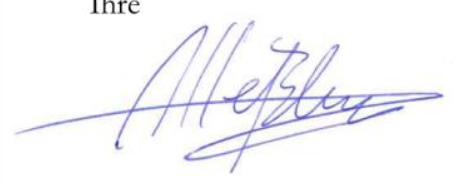

Dr. med. Arndt Heßling

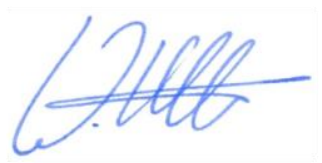

Prof. Dr. Wolfgang Himmel

Anlagen Fragebogen „Ambulante Ethikberatung bei Konflikten in der hausärztlichen Versorgung“ Kurz-Frage „Ich nehme an der Umfrage nicht teil, da ich ...“ 


\subsection{Fragebogen Nichtteilnahme}

\section{UNIVERSITÄTSMEDIZIN $=\mathbf{U M G}$
GÖTTINGEN $=\mathbf{O}$}

Ich nehme an der Umfrage nicht teil, da ich

口 im Moment keine Zeit habe.

$\square_{2} \quad$ zu oft mit Fragebögen konfrontiert werde und keine Ressourcen für deren Beantwortung aufwenden möchte.

$\square_{3}$ eine ambulante Ethikberatung nicht nutzen würde.

$\square_{4}$ sonstiges, und zwar: 


\subsection{Fragebogen Varel}

1. Wie oft sind Sie in Ihrer hausärztlichen Tätigkeit mit folgenden Entscheidungskonflikten konfrontiert?

\begin{tabular}{|c|c|c|c|c|c|}
\hline & $\begin{array}{l}\text { häufiger } \\
\text { als 1-mal } \\
\text { im Monat }\end{array}$ & $\begin{array}{l}\text { ca. 1-mal } \\
\text { im } \\
\text { Monat }\end{array}$ & $\begin{array}{l}\text { ca. 1-mal } \\
\text { im } \\
\text { Vierteljahr }\end{array}$ & $\begin{array}{l}\text { ca. 1-mal } \\
\text { im halben } \\
\text { Jahr }\end{array}$ & $\begin{array}{c}\text { seltener } \\
\text { als } 1 \text {-mal } \\
\text { im } \\
\text { halben } \\
\text { Jahr }\end{array}$ \\
\hline $\begin{array}{l}\text { Informationsweitergabe an Angehörige, } \\
\text { wenn nicht bekannt ist, ob der Patient dies } \\
\text { gestattet hat. }\end{array}$ & $\square_{1}$ & $\square_{2}$ & $\square_{3}$ & $\square_{4}$ & $\square_{5}$ \\
\hline $\begin{array}{l}\text { Nachträgliches Umsetzen einer } \\
\text { Patientenverfügung, die in einer } \\
\text { Notfallsituation nicht berücksichtig wurde. }\end{array}$ & $\boldsymbol{D}_{1}$ & $\square_{2}$ & $\square_{3}$ & $\square_{4}$ & $\square_{5}$ \\
\hline $\begin{array}{l}\text { Weitere Therapie, wenn die Wünsche des } \\
\text { Patienten aufgrund einer fortschreitenden } \\
\text { Erkrankung nicht feststellbar sind. }\end{array}$ & $\boldsymbol{\square}_{1}$ & $\boldsymbol{D}_{2}$ & $\square_{3}$ & $\square_{4}$ & $\square_{5}$ \\
\hline $\begin{array}{l}\text { Weitere Therapie, wenn eine potentiell } \\
\text { kurative Therapie von einem Patienten } \\
\text { schlecht vertragen wird. }\end{array}$ & $\square_{1}$ & $\square_{2}$ & $\square_{3}$ & $\square_{4}$ & $\square_{5}$ \\
\hline $\begin{array}{l}\text { Weitere Therapie, wenn eine } \\
\text { lebensverlängernde Therapie bei infauster } \\
\text { Prognose schlecht vertragen wird. }\end{array}$ & 口 & $\boldsymbol{D}_{2}$ & $\square_{3}$ & $\square_{4}$ & $\square_{5}$ \\
\hline $\begin{array}{l}\text { Einweisung von pflegebedürftigen o. } \\
\text { wesensveränderten Patienten in } \\
\text { Pflegeeinrichtungen gegen deren } \\
\text { vermeintlichen Willen. }\end{array}$ & $\boldsymbol{D}_{1}$ & $\boldsymbol{\square}_{2}$ & $\square_{3}$ & $\square_{4}$ & $\square_{5}$ \\
\hline $\begin{array}{l}\text { Selbstgefährdendes Patientenverhalten wie } \\
\text { beispielsweise Einstellung der } \\
\text { Nahrungsaufnahme o. eigene } \\
\text { Verwahrlosung. }\end{array}$ & $\boldsymbol{D}_{1}$ & $\square_{2}$ & $\square_{3}$ & $\square_{4}$ & $\square_{5}$ \\
\hline $\begin{array}{l}\text { Weiteres Vorgehen bei strafrechtlich } \\
\text { relevanten Informationen über Dritte wie } \\
\text { beispielsweise häusliche Gewalt durch den } \\
\text { Partner der Patientin. }\end{array}$ & $\boldsymbol{D}_{1}$ & $\boldsymbol{D}_{2}$ & $\square_{3}$ & $\square_{4}$ & $\square_{5}$ \\
\hline $\begin{array}{l}\text { Sinnhaftigkeit diagnostischer Maßnahmen } \\
\text { zur Früherkennung bei schwerkranken } \\
\text { Patienten. }\end{array}$ & $\boldsymbol{D}_{1}$ & $\boldsymbol{D}_{2}$ & $\square_{3}$ & $\square_{4}$ & $\square_{5}$ \\
\hline $\begin{array}{l}\text { Diagnostische oder therapeutische } \\
\text { Maßnahmen, wenn der Patientenwillen } \\
\text { nicht mit dem Willen der (pflegenden) } \\
\text { Angehörigen übereinstimmt. }\end{array}$ & $\boldsymbol{D}_{1}$ & $\boldsymbol{\square}_{2}$ & $\square_{3}$ & $\boldsymbol{\square}_{4}$ & $\square_{5}$ \\
\hline
\end{tabular}


2. Gibt es weitere Situationen, die Sie sehr konflikthaft wahrnehmen?

3. Nehmen Sie an Supervisions- oder Balintgruppen teil?

$\square_{1}$ ja

$\square_{2}$ nein

4. Sehen Sie Supervisions- oder Balintgruppen als hilfreich an, um Unterstützung in konflikthaften Situationen zu erhalten?
$\square_{1}$ ja
- bin mir nicht sicher
$\square_{3}$ nein

5. Haben Sie sich in einer der genannten Situationen oder darüber hinaus eine ambulante Ethikberatung gewünscht?
$\square_{1 j \mathrm{a}}$
$\square_{2}$ nein

6. Halten Sie die Etablierung einer ambulanten Ethikberatung für sinnvoll?
$\square_{1}$ ja
$\square_{2}$ bin mir nicht sicher
$\square_{3}$ nein

[falls nein, weiter mit Frage 11]

7. Welche Angebote sollte eine ambulante Ethikberatung umfassen?

(Mehrfachauswabl möglich)

$\square_{1}$ Telefonische Beratung

$\square_{2}$ Beratung in der Praxis

$\square_{4}$ retrospektive Fallbesprechung

$\square_{3}$ Beratung am Krankenbett

$\square_{5}$ Fortbildungen zu medizinethischen Fragestellungen für Hausärzte

口 Leitlinienerstellung für widerkehrende medizinische Konfliktsituation

口. Sonstige und zwar: 
8. Welche beruflichen Hintergründe sollten ambulante Ethikberater haben?

(Mehrfachauswabl möglich)

$\square_{1}$ Fachärztin/ Facharzt für Allgemeinmedizin $\quad \square_{2}$ Ärztinnen / Ärzte im allgemeinen

$\square_{3}$ Seelsorgerin /Seelsorger

$\square_{4}$ ambulante Pflegekräfte

$\square_{5}$ Sonstige und zwar:

9. Welche Qualifikationen und Eigenschaften sollten Ethikberater neben ihre Schulung zum Berater haben?

(Mehrfachauswabl möglich)

$\square_{1}$ über medizinisches Fachwissen verfügen

$\square_{2}$ über Erfahrungen in der ambulanten Patientenversorgung verfügen

$\square_{3}$ über Kenntnisse bezüglich des Medizinrechts verfügen

$\square_{4}$ andere Qualifikationen aufweisen und zwar:

10. Würden Sie eine ambulante Ethikberatung eher in Anspruch nehmen, wenn die aufgewendete Zeit vergütet werden würde?
$\square_{i}$ ja
$\square_{2}$ nein

11. Sie sind:
a weiblich
$\square_{2}$ männlich

12. Wie alt sind Sie?:

13. Haben Sie eine Zusatzqualifikation?

$\square_{1}$ ja, und zwar:

14. Seit wann arbeiten Sie in der ambulanten Patientenversorgung?

․ Seit

15. Sie arbeiten in einer
- Einzelpraxis
口 Gemeinschaftspraxis
口 Praxisgemeinschaft 


\subsection{Auszählung Hauptfragebogen}

1. Wie oft sind Sie in Ihrer hausärztlichen Tätigkeit mit folgenden Entscheidungskonflikten konfrontiert?

\begin{tabular}{|c|c|c|c|c|c|}
\hline & $\begin{array}{l}\text { ca. } 1-\mathrm{mal} \\
\text { im } \\
\text { Monat }\end{array}$ & $\begin{array}{l}\text { ca. } 1 \text {-mal } \\
\text { alle } \\
3 \text { Monate }\end{array}$ & $\begin{array}{l}\text { ca. } 1 \text {-mal } \\
\text { alle } \\
6 \text { Monate }\end{array}$ & $\begin{array}{l}\text { seltener als } \\
\text { alle } \\
6 \text { Monate }\end{array}$ & $\begin{array}{l}\text { bisher } \\
\text { nicht } \\
\text { erlebt }\end{array}$ \\
\hline \multicolumn{6}{|l|}{$n=453$} \\
\hline \multirow{3}{*}{$\begin{array}{l}\text { Fortsetzung einer potentiell kurativen } \\
\text { Therapie, die schlecht vertragen wird. }\end{array}$} & 208 & 99 & 71 & 63 & 12 \\
\hline & $\square_{5}$ & $\square_{4}$ & $\square_{3}$ & $\square_{2}$ & 口 \\
\hline & $45,9 \%$ & $21,9 \%$ & $15,7 \%$ & $13,9 \%$ & $2,7 \%$ \\
\hline \multicolumn{6}{|l|}{$n=455$} \\
\hline \multirow{3}{*}{$\begin{array}{l}\text { Fortsetzung einer potentiell } \\
\text { lebensverlängernden Therapie bei infauster } \\
\text { Prognose, die schlecht vertragen wird. }\end{array}$} & 83 & 106 & 111 & 124 & 31 \\
\hline & $\square_{5}$ & $\square_{4}$ & $\square_{3}$ & $\square_{2}$ & $\square_{1}$ \\
\hline & $18,2 \%$ & $23,3 \%$ & $24,4 \%$ & $27,3 \%$ & $6,8 \%$ \\
\hline \multicolumn{6}{|l|}{$n=455$} \\
\hline \multirow{3}{*}{$\begin{array}{l}\text { Fortsetzung einer Therapie, wenn der } \\
\text { Patientenwille wegen einer progredienten } \\
\text { Erkrankung nicht mehr feststellbar ist. }\end{array}$} & 47 & 67 & 93 & 193 & 55 \\
\hline & $\square_{5}$ & $\square_{4}$ & $\square_{3}$ & $\square_{2}$ & $\square_{1}$ \\
\hline & $10,3 \%$ & $14,7 \%$ & $20,4 \%$ & $42,4 \%$ & $12,1 \%$ \\
\hline \multicolumn{6}{|l|}{$n=456$} \\
\hline \multirow{3}{*}{$\begin{array}{l}\text { Informationsweitergabe an Angehörige, } \\
\text { wenn nicht bekannt ist, ob der Patient dies } \\
\text { gestattet hat. }\end{array}$} & 104 & 80 & 108 & 113 & 51 \\
\hline & $\square_{5}$ & $\square_{4}$ & $\square_{3}$ & $\square_{2}$ & $\square_{1}$ \\
\hline & $22,8 \%$ & $17,5 \%$ & $23,7 \%$ & $24,8 \%$ & $11,2 \%$ \\
\hline \multicolumn{6}{|l|}{$n=456$} \\
\hline \multirow{3}{*}{$\begin{array}{l}\text { Nachträgliches Umsetzen einer } \\
\text { Patientenverfügung, die in einer } \\
\text { Notfallsituation nicht berücksichtigt wurde. }\end{array}$} & 10 & 24 & 38 & 160 & 224 \\
\hline & $\square_{5}$ & $\square_{4}$ & $\square_{3}$ & $\square_{2}$ & $\square_{1}$ \\
\hline & $2,2 \%$ & $5,3 \%$ & $8,3 \%$ & $35,1 \%$ & $49,1 \%$ \\
\hline \multicolumn{6}{|l|}{$n=456$} \\
\hline \multirow{3}{*}{$\begin{array}{l}\text { Einweisung von pflegebedürftigen } o . \\
\text { wesensveränderten Patienten in } \\
\text { Pflegeeinrichtungen gegen deren Willen. }\end{array}$} & 13 & 49 & 102 & 222 & 70 \\
\hline & $\square_{5}$ & $\square_{4}$ & $\square_{3}$ & $\square_{2}$ & $\square_{1}$ \\
\hline & $2,9 \%$ & $10,8 \%$ & $22,4 \%$ & $48,7 \%$ & $15,4 \%$ \\
\hline \multicolumn{6}{|l|}{$n=453$} \\
\hline \multirow{3}{*}{ Selbstgefährdendes Patientenverhalten. } & 74 & 93 & 102 & 171 & 13 \\
\hline & $\square_{5}$ & $\square_{4}$ & $\square_{3}$ & $\square_{2}$ & $\square_{1}$ \\
\hline & $16,3 \%$ & $20,5 \%$ & $22,4 \%$ & $37,8 \%$ & $2,9 \%$ \\
\hline
\end{tabular}




\begin{tabular}{|c|c|c|c|c|c|}
\hline $\begin{array}{l}\mathrm{n}=454 \\
\text { Vorgehen bei strafrechtlich relevanten } \\
\text { Informationen über Dritte (beispielsweise } \\
\text { häusliche Gewalt durch den Partner). }\end{array}$ & $\begin{array}{c}6 \\
\square_{5} \\
1,3 \%\end{array}$ & $\begin{array}{l}23 \\
\square_{4} \\
5 \%\end{array}$ & $\begin{array}{c}53 \\
\square_{3} \\
11,7 \%\end{array}$ & $\begin{array}{c}254 \\
\square_{2} \\
56,0 \%\end{array}$ & $\begin{array}{c}118 \\
\boldsymbol{D}_{1} \\
26,0 \%\end{array}$ \\
\hline $\begin{array}{l}\mathrm{n}=456 \\
\text { Konträre Behandlungsansichten zwischen } \\
\text { mir und einer stationären Einrichtung. }\end{array}$ & $\begin{array}{c}94 \\
\square_{5} \\
20,6 \%\end{array}$ & $\begin{array}{c}123 \\
\boldsymbol{D}_{4} \\
27,0 \%\end{array}$ & $\begin{array}{c}87 \\
\square_{3} \\
19,1 \%\end{array}$ & $\begin{array}{c}116 \\
\boldsymbol{D}_{2} \\
25,4 \%\end{array}$ & $\begin{array}{c}36 \\
\square \\
7,1 \%\end{array}$ \\
\hline $\begin{array}{l}\mathrm{n}=455 \\
\text { Absetzen von Arzneien bei mittlerweile } \\
\text { fraglichem Nutzen für den Patienten. }\end{array}$ & $\begin{array}{c}235 \\
\boldsymbol{D}_{5} \\
51,7 \%\end{array}$ & $\begin{array}{c}129 \\
\square_{4} \\
28,4 \%\end{array}$ & $\begin{array}{c}67 \\
\square_{3} \\
14,7 \%\end{array}$ & $\begin{array}{c}20 \\
\boldsymbol{\square}_{2} \\
4,4 \%\end{array}$ & $\begin{array}{c}4 \\
\boldsymbol{\square}_{1} \\
0,9 \%\end{array}$ \\
\hline $\begin{array}{l}\mathrm{n}=456 \\
\text { Sinnhaftigkeit diagnostischer Maßnahmen } \\
\text { zur Früherkennung bei bereits } \\
\text { schwerkranken Patienten. }\end{array}$ & $\begin{array}{c}154 \\
\square_{5} \\
33,8 \%\end{array}$ & $\begin{array}{c}130 \\
\boldsymbol{口}_{4} \\
28,5 \%\end{array}$ & $\begin{array}{c}91 \\
\square_{3} \\
20,0 \%\end{array}$ & $\begin{array}{c}60 \\
\boldsymbol{D}_{2} \\
13,2 \%\end{array}$ & $\begin{array}{c}21 \\
\boldsymbol{口}_{1} \\
4,6 \%\end{array}$ \\
\hline $\begin{array}{l}\mathrm{n}=456 \\
\text { Patienten und Angehörigen sind uneins } \\
\text { bzgl. diagnostischer bzw. therapeutischer } \\
\text { Maßnahmen. }\end{array}$ & $\begin{array}{c}89 \\
\square_{5} \\
19,5 \%\end{array}$ & $\begin{array}{c}152 \\
\boldsymbol{口}_{4} \\
33,3 \%\end{array}$ & $\begin{array}{c}103 \\
\square_{3} \\
22,6 \%\end{array}$ & $\begin{array}{c}92 \\
\boldsymbol{口}_{2} \\
20,2 \%\end{array}$ & $\begin{array}{c}20 \\
\boldsymbol{口}_{1} \\
4,4 \%\end{array}$ \\
\hline Gibt es weitere Situationen, die a & Ihrer $\mathrm{Si}$ & konflik & sind? & & \\
\hline
\end{tabular}


3. Nehmen Sie an Qualitätszirkeln teil?

$\square_{1 \mathrm{ja}} \quad \square_{0}$ nein [falls nein, weiter mit Frage 5]

$298 \rightarrow 65,4 \% \quad 158 \rightarrow 34,7 \%$

4. Haben Sie in Qualitätszirkeln Unterstützung in konflikthaften Situationen erhalten?

$$
\mathrm{n}=294
$$

$\square_{1 j a} \quad \square_{2}$ war bisher nicht nötig $\quad \square_{0}$ nein

$160 \rightarrow 54,4 \% \quad 86 \rightarrow 29,3 \% \quad 48 \rightarrow 16,3 \%$

5. Nehmen Sie an Supervisions- oder Balintgruppen teil?

$\mathrm{n}=450$

$\square_{1}$ ja

$\square_{0}$ nein [falls nein, weiter mit Frage 7]

$96 \rightarrow 21,3 \%$

$354 \rightarrow 78,7 \%$

6. Haben Sie in Supervisions- oder Balintgruppen Unterstützung in konflikthaften Situationen erhalten?

$\mathrm{n}=95$
$\square_{1}$ ja
$\square_{2}$ war bisher nicht nötig
$\square_{0}$ nein
$81 \rightarrow 85,3 \%$
$10 \rightarrow 10,5 \%$
$4 \rightarrow 4,2 \%$

7. Haben Sie sich in einer der oben genannten Situationen oder bei vergleichbaren Konflikten eine ambulante Ethikberatung gewünscht? $\mathrm{n}=451$
$\square_{1} \mathrm{ja}$
$\square_{2}$ bin mir nicht sicher
$\square_{0}$ nein
$138 \rightarrow 30,6 \%$
$145 \rightarrow 32,2 \%$
$168 \rightarrow 37,3 \%$

8. Halten Sie die Etablierung einer ambulanten Ethikberatung für sinnvoll? $n=454$
$\square_{1 j a}$
$\square_{2}$ bin mir nicht sicher
$\square_{0}$ nein [falls nein, weiter mit Frage 13]
$245 \rightarrow 54,0 \%$
$160 \rightarrow 35,2 \%$
$49 \rightarrow 10,8 \%$ 
9. Welche Angebote sollte eine ambulante Ethikberatung umfassen? $\quad \mathrm{n}=401$

(Mehrfachauswabl möglich)

$\square_{1}$ telefonische Beratung $\quad \square_{2}$ Beratung in der Praxis

$327 \rightarrow 81,6 \% \quad 95 \rightarrow 23,7 \%$

$\square_{3}$ retrospektive Fallbesprechung

$\square$ Beratung am Krankenbett

$224 \rightarrow 55,9 \%$

$92 \rightarrow 22,9 \%$

$\square$ Fortbildungen zu medizinethischen Fragestellungen für Hausärzte

$285 \rightarrow 71,1 \%$

$\square_{6}$ Leitlinienerstellung für widerkehrende medizinische Konfliktsituation

$168 \rightarrow 41,9 \%$

$\square_{7}$ Sonstige und zwar

10. Welche beruflichen Hintergründe sollten ambulante Ethikberater haben?

(Mebrfachauswabl möglich)

$\mathrm{n}=401$

$\square_{1}$ Fachärztin/ Facharzt für Allgemeinmedizin

$\square_{2}$ Ärztinnen / Ärzte im Allgemeinen

$241 \rightarrow 60,1 \%$

$224 \rightarrow 55,9 \%$

$\square_{3}$ Seelsorgerin /Seelsorger

$\square_{4}$ ambulante Pflegekräfte

$185 \rightarrow 46,1 \%$

$112 \rightarrow 27,9 \%$

Sonstige und zwar: 
11. Welche Qualifikationen und Eigenschaften sollten Ethikberater neben ihrer Schulung zum Berater haben? $n=401$

(Mehrfachauswabl möglich)

$\square$ medizinisches Fachwissen

$357 \rightarrow 89,0 \%$

$\square_{2}$ Erfahrungen in der ambulanten Patientenversorgung

$359 \rightarrow 89,5 \%$

$\square_{3}$ Kenntnisse bezüglich des Medizinrechts

$347 \rightarrow 86,5 \%$

$\square_{4}$ andere Qualifikationen und zwar:

12. Würden Sie eine ambulante Ethikberatung eher in Anspruch nehmen, wenn die aufgewendete Zeit vergütet würde?

$\square_{1}$ ja

$\square_{2}$ bin mir nicht sicher

$\square_{0}$ nein

$99 \rightarrow 24,9 \%$ $108 \rightarrow 27,1 \%$ $191 \rightarrow 48,0 \%$

13. Sie sind: $\mathrm{n}=452$

a weiblich 口 männlich

$167 \rightarrow 37,0 \%$ $285 \rightarrow 63,0 \%$

14. Wie alt sind Sie? $\varnothing 54,0$ Jahre

15. Haben Sie Zusatzqualifikationen?

$\square_{1}$ ja, und zwar:

16. Seit wann arbeiten Sie in der ambulanten Patientenversorgung?
口. Seit
$\varnothing 18,7$ Jahren

17. Sie arbeiten in einer

口 ${ }_{1}$ Einzelpraxis

Gemeinschaftspraxis

$\mathrm{n}=449$

$168 \rightarrow 37,4 \%$

$234 \rightarrow 52,1 \%$

- Praxisgemeinschaft

$47 \rightarrow 10,5 \%$ 


\subsection{Auszählung Kurzfragebogen}

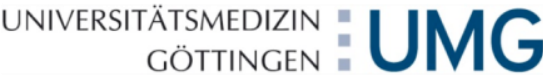

Ich nehme an der Umfrage nicht teil, da ich $n=119$

口 im Moment keine Zeit habe.

$62 \rightarrow 52,1 \%$

- zu oft mit Fragebögen konfrontiert werde und keine Ressourcen für deren Beantwortung aufwenden möchte.

$46 \rightarrow 38,7 \%$

$\square_{3}$ eine ambulante Ethikberatung nicht nutzen würde.

$23 \rightarrow 19,3 \%$

$\square_{4}$ sonstiges, und zwar: 


\subsection{Kodierung}

\section{Frage 1}

Wie oft sind Sie in Ihrer hausärztlichen Tätigkeit mit folgenden Entscheidungskonflikten konfrontiert?

\begin{tabular}{|c|c|c|}
\hline Kodierung & Name & Kodierung \\
\hline K_kurative_Th & $\begin{array}{l}\text { Fortsetzung einer potentiell } \\
\text { kurativen Therapie, die } \\
\text { schlecht vertragen wird. }\end{array}$ & $\begin{array}{l}\text { 5: ca. } 1 \text {-mal im Monat } \\
\text { 4: ca. } 1 \text {-mal alle } 3 \text { Monate } \\
\text { 3: ca. } 1 \text {-mal alle } 6 \text { Monate } \\
\text { 2: seltener als alle } 6 \text { Monate } \\
\text { 1: bisher nicht erlebt } \\
\text { 99: keine Antwort } \\
\text { 12: seltener als alle } 6 \text { Monate } \\
\text { 32: seltener als alle } 6 \text { Monate } \\
\text { 34: ca. } 1 \text {-mal alle } 3 \text { Monate } \\
\text { 345: ca. } 1 \text {-mal alle } 3 \text { Monate } \\
\text { 45: ca. } 1 \text {-mal alle } 3 \text { Monate }\end{array}$ \\
\hline K_infauste_P & $\begin{array}{l}\text { Fortsetzung einer potentiell } \\
\text { lebensverlängernden Thera- } \\
\text { pie bei infauster Prognose, } \\
\text { die schlecht vertragen wird. }\end{array}$ & s.o. \\
\hline K_Patientenwille & $\begin{array}{l}\text { Fortsetzung einer Therapie, } \\
\text { wenn der Patientenwille we- } \\
\text { gen einer progredienten Er- } \\
\text { krankung nicht mehr fest- } \\
\text { stellbar ist. }\end{array}$ & s.o. \\
\hline K_Information & $\begin{array}{l}\text { Informationsweitergabe an } \\
\text { Angehörige, wenn nicht be- } \\
\text { kannt ist, ob der Patient dies } \\
\text { gestattet hat. }\end{array}$ & s.o. \\
\hline K_Patientenverfuegung & $\begin{array}{l}\text { Nachträgliches Umsetzen } \\
\text { einer Patientenverfügung, die } \\
\text { in einer Notfallsituation nicht } \\
\text { berücksichtigt wurde. }\end{array}$ & s.o. \\
\hline K_Einweisung & $\begin{array}{l}\text { Einweisung von pflegebedürf- } \\
\text { tigen o. } \\
\text { wesensveränderten Patien- } \\
\text { ten in Pflegeeinrichtungen } \\
\text { gegen deren Willen. }\end{array}$ & s.o. \\
\hline K_Verhalten & $\begin{array}{l}\text { Selbstgefährdendes Patien- } \\
\text { tenverhalten. }\end{array}$ & s.o. \\
\hline
\end{tabular}




\begin{tabular}{|l|l|l|}
\hline K_Strafe & $\begin{array}{l}\text { Vorgehen bei strafrechtlich } \\
\text { relevanten Informationen } \\
\text { über Dritte (beispielsweise } \\
\text { häusliche Gewalt durch den } \\
\text { Partner). }\end{array}$ & \\
\hline K_Ansichten & $\begin{array}{l}\text { Konträre Behandlungsansich- } \\
\text { ten zwischen mir und einer } \\
\text { stationären Einrichtung. }\end{array}$ & S.o. \\
\hline K_Absetzen & $\begin{array}{l}\text { Absetzen von Arzneien bei } \\
\text { mittlerweile fraglichem Nut- } \\
\text { zen für den Patienten. }\end{array}$ & \\
\hline K_Frueherkennung & $\begin{array}{l}\text { Sinnhaftigkeit diagnostischer } \\
\text { Maßnahmen zur Früherken- } \\
\text { nung bei bereits schwerkran- } \\
\text { ken Patienten. }\end{array}$ & S.o. \\
\hline K_Angehoerige & $\begin{array}{l}\text { Patienten und Angehörigen } \\
\text { sind uneins bzgl. diagnosti- } \\
\text { scher bzw. therapeutischer } \\
\text { Maßnahmen. }\end{array}$ & \\
\hline
\end{tabular}

\section{Frage 2}

Weitere_K

Gibt es weitere Situationen, keine Antwort: 99

die Sie sehr konflikthaft wahrnehmen?

\section{Frage 3}

\begin{tabular}{|l|l|l|}
\hline Qualitaetszirkel & $\begin{array}{l}\text { Nehmen Sie an Qualitätszir- } \\
\text { keln teil? }\end{array}$ & $\begin{array}{l}\text { 1: ja } \\
\text { 0: nein } \\
\text { 99: keine Antwort }\end{array}$ \\
\hline
\end{tabular}

\section{Frage 4}

\begin{tabular}{|l|l|l|}
\hline Unterstuetzung_Q & Haben Sie in Qualitätszirkeln & 1: ja \\
& Unterstützung in konflikthaf- & 2: war bisher nicht nötig \\
& ten Situationen erhalten? & 0: nein \\
& 99: keine Antwort \\
& $\begin{array}{l}\text { 77: Frage korrekterweise nicht } \\
\text { beantwortet oder Antwort nicht } \\
\end{array}$ \\
& relevant \\
\hline
\end{tabular}

\section{Frage 5}

\begin{tabular}{|l|l|l|}
\hline Supervisionsgruppe & $\begin{array}{l}\text { Nehmen Sie an Supervisi- } \\
\text { ons- oder Balintgruppen teil? }\end{array}$ & $\begin{array}{l}\text { 0: nein } \\
\text { 99: keine Antwort }\end{array}$ \\
\hline
\end{tabular}




\section{Frage 6}

\begin{tabular}{|l|l|l|}
\hline Unterstuetzung_S & Haben Sie in Supervisions- & 1: ja \\
& oder Balintgruppen Unter- & 2: war bisher nicht nötig \\
& Stützung in konflikthaften & S: nein \\
& Situationen erhalten? & 0: \\
& 99: keine Antwort \\
& 77: Frage korrekterweise nicht \\
& beantwortet oder Antwort nicht \\
relevant
\end{tabular}

\section{Frage 7}

\begin{tabular}{|l|l|l|}
\hline Wunsch_EB & Haben Sie sich in einer der & 1: ja \\
& oben genannten Situationen & 2: bin mir nicht sicher \\
& oder bei vergleichbaren Kon- & flikten eine ambulante Ethik- \\
& 0: nein \\
& beratung gewünscht? & 99: keine Antwort \\
\hline
\end{tabular}

\section{Frage 8}

\begin{tabular}{|l|l|l|}
\hline EB_Sinnvoll & Halten Sie eine Etablierung & 1: ja \\
& einer ambulanten Ethikbera- & 2: bin mir nicht sicher \\
& tung für sinnvoll? & 0: nein \\
& & 99: keine Antwort \\
\hline
\end{tabular}

\section{Frage 9}

Welche Angebote sollte eine Ethikberatung umfassen?

\begin{tabular}{|c|c|c|}
\hline A_Telefon & telefonische Beratung & $\begin{array}{l}\text { 1: ja } \\
\text { 0: nein } \\
\text { 99: keine Antwort } \\
\text { 77: Frage korrekterweise nicht } \\
\text { beantwortet oder Antwort nicht } \\
\text { relevant }\end{array}$ \\
\hline A_Praxis & Beratung in der Praxis & s.o. \\
\hline A_Fallbesprechung & retrospektive Fallbesprechung & S.o. \\
\hline A_Krankenbett & Beratung am Krankenbett & s.o. \\
\hline A_Fortbildung & $\begin{array}{l}\text { Fortbildungen zu medizinethi- } \\
\text { schen Fragestellungen für } \\
\text { Hausärzte }\end{array}$ & s.o. \\
\hline A_Leitlinien & $\begin{array}{l}\text { Leilinienerstellung für wieder- } \\
\text { kehrende medizinische Kon- } \\
\text { fliktsituation }\end{array}$ & s.o. \\
\hline A_Sonstige & Sonstige und zwar & keine Antwort: 99 \\
\hline
\end{tabular}




\section{Frage 10}

Welche beruflichen Hintergründe sollten ambulante Ethikberater haben?

\begin{tabular}{|l|l|l|}
\hline B_Allgemeinarzt & $\begin{array}{l}\text { Fachärztin/ Facharzt für All- } \\
\text { gemeinmedizin }\end{array}$ & $\begin{array}{l}\text { 1: ja } \\
\text { 0: nein } \\
\text { 99: keine Antwort } \\
\text { 77: Frage korrekterweise nicht } \\
\text { beantwortet oder Antwort nicht } \\
\text { relevant }\end{array}$ \\
\hline B_Arzt & $\begin{array}{l}\text { Ärztinnen / Ärzte im Allgemei- } \\
\text { nen }\end{array}$ & \begin{tabular}{l} 
S.o. \\
\hline B_Seelsorger
\end{tabular} \\
\hline B_Pflege & Seelsorgerin / Seelsorger & S.o. \\
\hline B_Sonstige & ambulante Pflegekräfte & s.o. \\
\hline
\end{tabular}

\section{Frage 11}

Welche Qualifikationen und Eigenschaften sollten Ethikberater neben ihrer Schulung zum Berater haben?

\begin{tabular}{|l|l|l|}
\hline Q_Fachwissen & medizinisches Fachwissen & $\begin{array}{l}\text { 1: ja } \\
\text { 0: nein } \\
\text { 99: keine Antwort } \\
\end{array}$ \\
& & $\begin{array}{l}\text { 77: Frage korrekterweise nicht } \\
\text { beantwortet oder Antwort nicht } \\
\text { relevant }\end{array}$ \\
\hline Q_Erfahrung & $\begin{array}{l}\text { Erfahrungen in der ambulanten } \\
\text { Patientenversorgung }\end{array}$ & s.o. \\
\hline Q_Medizinrecht & $\begin{array}{l}\text { Kenntnisse bezüglich des Me- } \\
\text { dizinrechts }\end{array}$ & s.o. \\
\hline Q_Andere & $\begin{array}{l}\text { andere Qualifikationen und } \\
\text { zwar: }\end{array}$ & 99: keine Antwort \\
\hline
\end{tabular}

\section{Frage 12}

\begin{tabular}{|l|l|l|}
\hline Verguetung & Würden Sie eine ambulante & 1: ja \\
& $\begin{array}{l}\text { Ethikberatung eher in An- } \\
\text { spruch nehmen, wenn die auf- } \\
\text { gewendete Zeit vergütet wür- } \\
\text { de? }\end{array}$ & 0: nein mir nicht sicher \\
& 12: bin mir nicht sicher \\
& 99: keine Antwort \\
& 77: Frage korrekterweise nicht \\
& beantwortet oder Antwort nicht \\
& relevant \\
\hline
\end{tabular}


Frage 13

\begin{tabular}{|l|l|l|}
\hline Geschlecht & Sie sind: & $\begin{array}{l}\text { 1: weiblich } \\
\text { 2: männlich }\end{array}$ \\
\hline
\end{tabular}

\section{Frage 14}

\begin{tabular}{|l|l|l|}
\hline Alter & Wie alt sind Sie? & Antwort wurde als Zahl eingege- \\
& & ben \\
& & ü60: 62 Jahre \\
& 99: keine Antwort \\
\hline
\end{tabular}

Frage 15

\begin{tabular}{|l|l|l|}
\hline Zusatzqualifikation & $\begin{array}{l}\text { Haben Sie Zusatzqualifikatio- } \\
\text { nen? }\end{array}$ & $\begin{array}{l}\text { Antwort wurde eingegeben } \\
\text { 99: keine Zusatzqualifikation }\end{array}$ \\
\hline
\end{tabular}

\section{Frage 16}

\begin{tabular}{|l|l|l|}
\hline Patientenversorgung & $\begin{array}{l}\text { Seit wann arbeiten Sie in der } \\
\text { ambulanten Patientenversor- } \\
\text { gung? }\end{array}$ & $\begin{array}{l}\text { Antwort wurde als Zahl eingege- } \\
\text { ben } \\
\text { 99: keine Antwort }\end{array}$ \\
\hline
\end{tabular}

\section{Frage 17}

\begin{tabular}{|c|c|c|}
\hline Praxisart & Sie arbeiten in einer: & $\begin{array}{l}\text { 1: Einzelpraxis } \\
\text { 2: Gemeinschaftspraxis } \\
\text { 3: Praxisgemeinschaft } \\
\text { 4: MVZ, wird zu ,3' Praxisge- } \\
\text { meinschaft gezählt } \\
\text { 23: Gemeinschaftspraxis } \\
\text { 99: Keine Antwort }\end{array}$ \\
\hline
\end{tabular}




\section{Danksagung}

Ich danke meinem Doktorvater Herrn Prof. Dr. disc. pol. W. Himmel für hervorragende fachliche Betreuung bei der Erstellung dieser Dissertation und die vielen, stets sinnvollen Anregungen.

Weiterhin danke ich allen Mitarbeitern und ehemaligen Mitarbeitern des Instituts für Allgemeinmedizin der Universität Göttingen - insbesondere Dr. med. Arndt Heßling - für die kollegiale Unterstützung und die konstruktive Kritik bei der Erstellung dieser Arbeit.

Markus Harden, Doktorand in der Abteilung für Medizinische Statistik der Universität Göttingen, gilt mein Dank für die Hilfe bei der Lösung statistischer Probleme.

Mein besonderer Dank gilt den Hausärztinnen und Hausärzten für die Teilnahme an dieser Studie. 